\title{
Precision Distances to Dwarf Galaxies and Globular Clusters from Pan-STARRS1 $3 \pi$ RR Lyrae
}

\author{
Nina Hernitschek ${ }^{1}\left(\mathbb{D}\right.$, Judith G. Cohen ${ }^{1}(\mathbb{D})$, Hans-Walter Rix ${ }^{2}\left(\mathbb{D}\right.$, Eugene Magnier ${ }^{3}(\mathbb{1})$, Nigel Metcalfe ${ }^{4}$, Richard Wainscoat ${ }^{3}$ (iD), \\ Christopher Waters ${ }^{5}$, Rolf-Peter Kudritzki ${ }^{3}$, and William Burgett ${ }^{6}$ \\ ${ }^{1}$ Division of Physics, Mathematics and Astronomy, Caltech, Pasadena, CA 91125, USA; ninah@astro.caltech.edu \\ ${ }_{2}^{2}$ Max-Planck-Institut für Astronomie, Königstuhl 17, D-69117 Heidelberg, Germany \\ ${ }^{3}$ Institute for Astronomy, University of Hawaii at Manoa, Honolulu, HI 96822, USA \\ ${ }^{4}$ Department of Physics, University of Durham, South Road, Durham DH1 3LE, UK \\ ${ }^{5}$ Department of Astrophysical Sciences, 4 Ivy Lane, Princeton University, Princeton, NJ 08544, USA \\ ${ }^{6}$ GMTO Corporation (Pasadena), 465 N. Halstead Street, Suite 250, Pasadena, CA 91107, USA \\ Received 2018 September 21; revised 2018 November 19; accepted 2018 November 20; published 2019 January 21
}

\begin{abstract}
We present new spatial models and distance estimates for globular clusters and dwarf spheroidals orbiting our Galaxy based on RR Lyrae (RRab) stars in the Pan-STARRS1 (PS1) $3 \pi$ survey. Using the PS1 sample of RRab stars from Sesar et al. in 16 globular clusters and 5 dwarf galaxies, we fit structural models in $(l, b, D)$ space; for 13 globular clusters and 6 dwarf galaxies, we give only their mean heliocentric distance $D$. We verify the accuracy of the period-luminosity relations used in Sesar et al. to constrain the distance to those stars, and compare them to period-luminosity-metallicity relations using metallicities from Carretta et al. We compare our Sesar et al. distances to the parallax-based Gaia DR2 distance estimates from Bailer-Jones et al. and find our distances to be consistent and considerably more precise.
\end{abstract}

Key words: Galaxy: halo - globular clusters: general

\section{Introduction}

In this paper, we exploit the opportunities provided by the Pan-STARRS1 (PS1) 3 $\pi$ RR Lyrae catalog (Sesar et al. 2017) to explore the Galactic halo. Using that large data set of RRab stars covering three quarters of the sky out to more than $130 \mathrm{kpc}$, in Hernitschek et al. (2018) we looked at the seemingly smooth part of the stellar halo. We now focus on the RRab stars in globular clusters (GC) and dwarf galaxies in order to determine their distances and constrain their structure. For most of these objects, RRab stars may be the most precise distance tracers. As shown in Section 8, all GC and dwarf galaxies we study here are beyond any parallax precision of Gaia DR2, which is according to Bailer-Jones et al. (2018) only valid within a heliocentric distance range of about $5 \mathrm{kpc}$. The main advantages of such a study are that due to the large angular extent and depth of PS1 $3 \pi$, the source of observational data as well as the methodology are the same for all GC and dwarf spheroidal (dSph) galaxies we analyze. The aim of this paper is to precisely constrain the position and extent of each of these overdensities from RRab stars, where we determine these properties for $29 \mathrm{GC}$ and $11 \mathrm{dSph}$ galaxies, and if this is not possible, to at least give their mean heliocentric distances. Furthermore, from the globular clusters we demonstrate that the period-luminosity relations used by Sesar et al. (2017) to determine the distances of the RRab stars are precise and their predicted magnitudes are well within the claimed uncertainties.

RR Lyrae stars (RRL) as distance indicators have the advantage of being low-mass stars found in both early- and late-type stellar systems (van den Bergh 1999). When periods, or periods and metallicities, for RRL are known, their distances can be estimated using period-luminosity (PL) or periodluminosity-metallicity (PLZ) relations. Visual magnitudemetallicity relations are described for example in Chaboyer et al. (1996), Bono et al. (2003), and Cacciari \& Clementini (2003). In the infrared, the extinction is smaller and the amplitude of variation is smaller. In the near-infrared (NIR), well-defined PL relations can be found (Longmore et al. 1986; Bono et al. 2001, 2003; Catelan et al. 2004; Braga et al. 2015; Sesar et al. 2017), extending to the midinfrared with only small scatter (Madore et al. 2013; Klein et al. 2014).

These individual distance estimates can then be used to calculate distances to and the shape of globular clusters as well as dwarf galaxies. Dwarf galaxies orbiting our Milky Way are not only interesting objects per se, but can also provide us with important information to determine the Milky Way's total mass and dynamics. Dambis et al. (2013) used WISE as well as zeropoints from $H S T$ parallaxes to derive PL relations from 360 RRL belonging to 15 globular clusters.

More recent work on the distances of globular clusters was carried out by Neeley et al. (2015) who investigated mid-IR PLZ relations for the globular cluster NGC 6121 (M4), which is not in our sample because it lies outside of the PS1 $3 \pi$ footprint. Their work contains PL relations for both the RRab and RRc samples as well as for the combined RRab+RRc sample. In their 2017 paper, Neeley et al. (2017) also present theoretical mid-IR PLZ relations for RR Lyrae stars at Spitzer and WISE wavelengths and show that the mid-IR PLZ relations can provide distance estimates to individual RR Lyrae stars with uncertainties better than $2 \%$. This is comparable to the result by Sesar et al. (2017) derived for the RRab stars with optical photometry used in this paper. For the dSph Sculptor, which lies outside of the PS1 $3 \pi$ footprint, Garofalo et al. (2018) derived mid-IR PLZ relations and calculated the distance to the dwarf galaxy as part of the Spitzer SHMASH project, which targeted in total four dSph (Ursa Minor dSph, Bootes dSph, Sculptor dSph, and Carina dSph).

This paper is structured as follows. In Section 2, we introduce the PS1 $3 \pi$ survey and lay out the properties of the PS1 RRab stars. This is followed in Section 3 by a description of the spatial area covered by PS1 $3 \pi$, and the list of GCs and dSph that are 
included in it. In Section 4, we present the method of fitting a series of parameterized stellar density models and the probabilistic approach to constrain their model parameters. We describe fitting tests on mock data in Section 4.3. In Section 5, the position and extent are estimated for each $\mathrm{GC}$ and $\mathrm{dSph}$. We present various analyses based on these fitted parameters. In Section 6, we calculate mean distances for those dwarf galaxies and GC, which do not have enough sources for the fitting process. We also discuss the implications on PL relations in Section 7 and compare our distance estimates to those derived using the second Gaia data release in Section 8. A discussion and summary of our results is given in Section 9. The main part of the paper is followed by a large Appendix containing figures as well as tables.

\section{RR Lyrae Stars from the PS1 Survey}

As in our previous papers, e.g., Hernitschek et al. (2017) and Hernitschek et al. (2018), our analysis is based on a sample of highly likely RRab stars, as selected by Sesar et al. (2017) from the Pan-STARRS1 $3 \pi$ survey. Here, we briefly describe the pertinent properties of the PS1 $3 \pi$ survey and the RR Lyrae light curves obtained, and recapitulate briefly the process of selecting the likely RRab for the catalog of PS1 RRab stars, as laid out in Sesar et al. (2017). We also briefly characterize the obtained candidate sample.

The Pan-STARRS 1 (PS1) survey (Kaiser et al. 2010) collected multi-epoch, multicolor observations undertaking a number of surveys, among which the PS1 $3 \pi$ survey (Chambers et al. 2016) is currently the largest. It has observed the entire sky north of decl. $-30^{\circ}$ in five filter bands $\left(g_{\mathrm{P} 1}, r_{\mathrm{P} 1}, i_{\mathrm{P} 1}, z_{\mathrm{P} 1}, y_{\mathrm{P} 1}\right)$ with a $5 \sigma$ single epoch depth of about 22.0, 22.0, 21.9, 21.0, and $19.8 \mathrm{mag}$ in $g_{\mathrm{P} 1}, r_{\mathrm{P} 1}, i_{\mathrm{P} 1}, z_{\mathrm{P} 1}$, and $y_{\mathrm{P} 1}$, respectively (Stubbs et al. 2010; Tonry et al. 2012).

Starting with a sample of more than $1.1 \times 10^{9}$ PS1 $3 \pi$ sources, Hernitschek et al. (2016) and Sesar et al. (2017) subsequently selected a sample of 44,403 likely RRab stars, of which $\sim 17,500$ are at $R_{\mathrm{gc}} \geqslant 20 \mathrm{kpc}$, by applying machinelearning techniques based on light-curve characteristics. RRab stars are the most common type of RR Lyrae, making up $91 \%$ of all observed RR Lyrae (Smith 2004), and displaying the steep rises in brightness typical of RR Lyrae.

The identification of the RRab stars is highly effective, and the sample of RRab stars is pure $(90 \%)$ and complete $(\geqslant 80 \%$ at $80 \mathrm{kpc}$ ) at high galactic latitudes. Distances to these stars were calculated based on flux-averaged $i_{\mathrm{P} 1}$ magnitudes, corrected for dust extinction using extinction coefficients of Schlafly \& Finkbeiner (2011) and the dust map of Schlafly et al. (2014). The distance estimates are precise to $3 \%$, based on newly derived PL relations for the optical/near-infrared PS1 bands (Sesar et al. 2017). Overall, this results in the widest (three quarters of the sky) and deepest (reaching $>120 \mathrm{kpc}$ ) sample of RR Lyrae stars to date, allowing us to observe them globally across the Milky Way. Out of these sources, 1093 exist beyond a Galactocentric distance of $80 \mathrm{kpc}$, with 238 beyond $100 \mathrm{kpc}$, which enables us to estimate distances to and extents of dwarf galaxies and globular clusters. RRc stars, which were also selected by Sesar et al. (2017), are not included in our study.

\section{Sample of Globular Clusters and Dwarf Galaxies}

PS1 $3 \pi$, as it covers the entire sky north of decl. $-30^{\circ}$ out to more than $130 \mathrm{kpc}$, gives us access to many globular clusters (GC) and several dwarf galaxies.
Table 1

Dwarf Galaxies, Attempted

\begin{tabular}{lrrr}
\hline \hline Name & $\left(l_{\text {prior }}\left[{ }^{\circ}\right]\right.$, & $b_{\text {prior }}\left[{ }^{\circ}\right]$, & $\left.D_{\text {prior }}[\mathrm{kpc}]\right)$ \\
\hline Aquarius II dSph & 55.0 & -53.0 & 108 \\
Bootes I dSph & 358.036 & 69.642 & 60 \\
Crater II dSph & 282.9084 & 42.0276 & 117.5 \\
Draco dSph & 86.3747 & 34.7171 & 79 \\
Sagittarius dSph & 5.6081 & -14.0858 & 26.3 \\
Segue 1 dSph & 220.488 & 50.420 & 23 \\
Segue 2 dSph & 149.433 & -38.1352 & 35 \\
Sextans dSph & 243.4981 & 42.2721 & 86 \\
Ursa Minor Dwarf dE4 & 104.9527 & 44.8028 & 63 \\
Ursa Major I dSph & 159.4311 & 54.4143 & 100 \\
Ursa Major II Dwarf & 152.464 & 37.443 & 30 \\
\hline
\end{tabular}

Note. The list of dwarf galaxies within the PS1 $3 \pi$ footprint for which we want to determine their distance. Their $(l, b, D)$ coordinates are from McConnachie (2012).

To select those overdensities for which fitting their density might be feasible, we started with a list of dwarf galaxies within $3 \mathrm{Mpc}$ by McConnachie (2012), its update from $2014^{7}$ and a list of currently known globular clusters from the 2010 update of Harris (1996). We excluded the ones outside the PS1 $3 \pi$ footprint and made plots of regions on the sky around for each of the remaining ones. If an overdensity is apparent in the PS1 RRab sample from visual inspection, we consider it for further analysis. We ended up with a list of 11 dwarf galaxies and 29

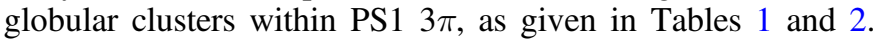
Plots of a subset of these overdensities are found in the Appendix, Figures 1-11. For plotting those overdensities, we chose a Cartesian projection of $(l, b)$ to more easily compare to their marginalized distributions in $l$ and $b$ (histograms). With this choice, high-latitude overdensities such as NGC 5024, NGC 5053, and NGC 5272 appear to be elongated in the $l$ direction.

We expected that the central regions of the globular clusters might not be well represented due to crowding and blending effects that made many RRab stars fail to meet the criteria for inclusion in the catalog of PS1 RRab stars (Sesar et al. 2017). To test that, we compared our sample with the Catalog of Variable Stars in Globular Clusters from Clement et al. (2001). There are 11 globular clusters detectable with RRab stars both in the catalog of PS1 RRab stars and the Catalog of Variable Stars in Globular Clusters. As shown in Figure 12, we find that for each of the 11 globular clusters available in both catalogs, our catalog misses most RRab in the globular cluster's central regions. The $\mathrm{dSph}$ galaxies are sufficiently diffuse that this is not a significant issue.

\section{Density Fitting}

In this section, we lay out a forward-modeling approach to describe the spatial distribution of RRab stars within and near overdensities such as dwarf galaxies and globular clusters. We are using a local halo model describing the background of field stars from the halo as in Hernitschek et al. (2018) and a smooth spheroidal distribution describing the overdensity itself. Regarding the dwarf galaxies, this approach is similar to Martin et al. (2008), who derived structural parameters of Milky Way satellites from SDSS data.

\footnotetext{
https://www.astrosci.ca/users/alan/Nearby_Dwarfs_Database.html
} 
Table 2

Globular Clusters, Attempted

\begin{tabular}{|c|c|c|c|}
\hline Name & $\left(l_{\text {prior }}\left[{ }^{\circ}\right]\right.$, & $b_{\text {prior }}\left[{ }^{\circ}\right]$, & $\left.D_{\text {prior }}[\mathrm{kpc}]\right)$ \\
\hline IC 1257 & 16.54 & 15.15 & 25 \\
\hline NGC 2419 & 180.37 & 25.24 & 82.6 \\
\hline NGC 4147 & 252.85 & 77.19 & 19.3 \\
\hline NGC 4590 (M68) & 299.63 & 36.05 & 10.3 \\
\hline NGC 5024 (M53) & 332.96 & 79.76 & 17.9 \\
\hline NGC 5053 & 335.7 & 78.95 & 17.4 \\
\hline NGC 5272 (M3) & 42.22 & 78.71 & 10.2 \\
\hline NGC 5466 & 42.15 & 73.59 & 16 \\
\hline NGC 5634 & 342.21 & 49.26 & 25.2 \\
\hline NGC 5694 & 331.06 & 30.36 & 35 \\
\hline NGC 5897 & 342.95 & 30.29 & 12.5 \\
\hline NGC 5904 (M5) & 3.86 & 46.8 & 7.5 \\
\hline NGC 6093 (M80) & 352.67 & 19.46 & 10 \\
\hline NGC 6171 (M107) & 3.37 & 23.01 & 5.4 \\
\hline NGC 6229 & 73.64 & 40.31 & 30.5 \\
\hline NGC 6356 & 6.72 & 10.22 & 15.1 \\
\hline NGC 6402 (M14) & 21.32 & 14.81 & 9.3 \\
\hline NGC 6426 & 28.09 & 16.23 & 20.6 \\
\hline NGC 6864 (M75) & 20.3 & -25.75 & 20.9 \\
\hline NGC 6934 & 52.1 & -18.89 & 15.6 \\
\hline NGC 6981 (M72) & 35.16 & -32.68 & 17 \\
\hline NGC 7006 & 63.77 & -19.41 & 41.2 \\
\hline NGC 7078 (M15) & 65.01 & -27.31 & 10.4 \\
\hline NGC 7089 (M2) & 53.37 & -35.77 & 11.5 \\
\hline NGC 7099 (M30) & 27.18 & -46.84 & 8.1 \\
\hline Pal 1 & 130.06 & 19.03 & 11.1 \\
\hline Pal 3 & 240.15 & 41.86 & 92.5 \\
\hline Pal 5 & 0.85 & 45.86 & 23.2 \\
\hline Pal 13 & 87.1 & -42.7 & 26 \\
\hline
\end{tabular}

Note. The list of globular clusters within the PS1 $3 \pi$ footprint for which we want to determine their distance. Their $(l, b, D)$ coordinates are from a current update of Harris (1996).

We presume that the local stellar halo distribution can be sensibly approximated by a spheroidal distribution with a parameterized radial profile (see also Hernitschek et al. 2018). The overdensities can be described by multivariate Gaussians in $(l, b, D)$. Following a number of previous studies (e.g., Sesar et al. 2013; Cohen et al. 2015; Xue et al. 2015; Iorio et al. 2017; Hernitschek et al. 2018), we presume that the overall radial density profile of the halo outside of overdensities-the distance-dependent "background" of field stars in a given direction-can be described by a power-law profile. We attempt to carry out the fit on small $5 \times 5 \mathrm{deg}^{2}$ patches on the sky around the assumed position prior $\left(l_{\text {prior }}, b_{\text {prior }}\right)$. We thus neglect the selection effects and halo flattening described in Hernitschek et al. (2018) and instead fit for a local stellar halo distribution as a function of a power-law index $n$ only.

We apply a forward-modeling process to fit stellar-density models to the data by generating the expected observed distribution of stars in the RRab sample, based on our model for the halo background and overdensity. The predicted distribution is then automatically compared to the observed star counts to calculate the likelihood.

\subsection{Stellar Density Model}

For the overall radial density profile of the halo stars, we assume a power-law model

$$
\rho_{\text {halo }}(X, Y, Z)=\rho_{\odot \mathrm{RRL}}\left(R_{\odot} / r_{q}\right)^{n}
$$

where $\rho_{\odot \text { RRL }}$ gives the number density of RR Lyrae at the position of the Sun, $n$ is the power-law index where larger values of $n$ indicate a steeper profile, $R_{\odot}$ is the distance of the Sun from the Galactic center, and $r_{q}=\sqrt{\left(X^{2}+Y^{2}+(Z / q)^{2}\right)}$ is the flattening-corrected radius.

In Hernitschek et al. (2018), we derived the halo flattening on concentric ellipsoids. However, as we deal here with very small patches on the sky, and do not want to derive a reliable fit for the halo but want to remove any background distribution, we decided to set $q=0.75$, leaving $n$ the only free parameter for the halo component. Also, we are not fitting for $\rho_{\odot \mathrm{RRL}}$.

The spatial density of an overdensity, i.e., a dwarf galaxy or globular cluster, is described by a multivariate Gaussian $\mathcal{G}$ :

$$
\begin{aligned}
\rho_{*}(l, b, D)=\mathcal{G}(l, b, D) \\
=\frac{\exp \left(-0.5\left[\left(\frac{l-\bar{l}}{\sigma_{l}}\right)^{2}+\left(\frac{b-\bar{b}}{\sigma_{b}}\right)^{2}+\left(\frac{D-\bar{D}}{\sigma_{D}}\right)^{2}\right]\right)}{\sigma_{l} \sigma_{b} \sigma_{D}(2 \pi)^{(3 / 2)}} .
\end{aligned}
$$

The data set $\mathcal{D}$ is given as $\mathcal{D}=(D, \delta D, l, b)$. The parameters are $\boldsymbol{\theta}=\left(\bar{l}, \bar{b}, \bar{D}, \sigma_{l}, \sigma_{b}, \sigma_{D}, f_{*}, n\right)$, composed of the spatial position of the overdensity $(\bar{l}, \bar{b}, \bar{D})$, its extent $\left(\sigma_{l}, \sigma_{b}, \sigma_{D}\right)$, and the fraction of the stars $f_{*}$ in the overdensity, and the power-law index $n$ of the halo model. The heliocentric distance distribution of stars is then characterized by

$$
\begin{aligned}
p_{\mathrm{RRL}}(\mathcal{D} \mid \boldsymbol{\theta})= & p_{\text {halo }}(\mathcal{D} \mid \boldsymbol{\theta})+p_{*}(\mathcal{D} \mid \boldsymbol{\theta}) \\
= & \left(1-f_{*}\right) \times \hat{\rho}_{\text {halo }}(l, b, D, n) \\
& +f_{*} \times \hat{\rho}_{*}\left(l, b, D, \bar{l}, \bar{b}, \bar{D}, \sigma_{l}, \sigma_{b}, \sigma_{D}\right),
\end{aligned}
$$

where

$$
\hat{\rho}_{\text {halo }}(l, b, D, q, n) \equiv \frac{\rho_{\text {halo }}(l, b, D, q, n)}{\int \rho_{\text {halo }}(l, b, D, q, n) d D},
$$

with an analogous definition of $\hat{\rho}_{*}$.

Although we were aware of a distance uncertainty of $3 \%$ for RRab stars from Sesar et al. (2017), we have not included it in our calculations yet. The reason for this is that the distance uncertainty can easily be taken into account later on, as the distance uncertainty $\epsilon_{D} \sim 0.03 D$ adds in quadrature to the (true) width in distance:

$$
\sigma_{D}=\sqrt{\left(\sigma_{D}^{\prime}\right)^{2}+\epsilon_{D}^{2}} .
$$

We will deal with the distance precision versus true line-ofsight depth later when we evaluate our fitting results.

\subsection{Constraining Model Parameters}

With the model $\rho_{\mathrm{RRL}}(\mathcal{D} \mid \boldsymbol{\theta})$ at hand, we can directly calculate the likelihood of the data $\mathcal{D}$ given the model $\rho_{\mathrm{RRL}}$ and the fitting parameters $\boldsymbol{\theta}$.

The normalized unmarginalized logarithmic likelihood for the $i$ th star with the observables $\mathcal{D}_{i}$ is then

$$
\ln p\left(\mathcal{D}_{i} \mid \boldsymbol{\theta}\right)=\frac{\rho_{\mathrm{RRL}}\left(\mathcal{D}_{i} \mid \boldsymbol{\theta}\right)|\boldsymbol{J}|}{\iiint \rho_{\mathrm{RRL}}(l, b, D \mid \boldsymbol{\theta})|\boldsymbol{J}| d l d b d D},
$$

where the normalization integral is over the observed volume. The Jacobian term $|\boldsymbol{J}|=D^{2} \cos b$ reflects the transformation from $(X, Y, Z)$ to $(l, b, D)$ coordinates.

We evaluate the logarithmic posterior probability of the parameters $\boldsymbol{\theta}$ of the halo model, given the full data $\mathcal{D}$ and a 
prior $p(\boldsymbol{\theta}), \ln p(\boldsymbol{\theta} \mid \mathcal{D})=\ln p(\mathcal{D} \mid \boldsymbol{\theta})+\ln p(\boldsymbol{\theta})$ with

$$
\ln p(\mathcal{D} \mid \boldsymbol{\theta})=\sum_{i} \ln p\left(\mathcal{D}_{i} \mid \boldsymbol{\theta}\right)
$$

being the marginal log likelihood for the full data set.

To determine the best-fit parameters and their uncertainties, we sample the posterior probability over the parameter space with Goodman \& Weare's Affine Invariant Markov Chain Monte Carlo (Goodman \& Weare 2010), making use of the Python module emcee (Foreman-Mackey et al. 2013).

The final best-fit values of the model parameters have been estimated using the median of the posterior distributions; the uncertainties have been estimated using the 15.87th and 84.13th percentiles. For a parameter whose pdf can be welldescribed by a Gaussian distribution, the difference between the 15.87 th and 84.13 th percentile is equal to $1 \sigma$.

Our model has eight free parameters, $\boldsymbol{\theta}=(\bar{l}, \bar{b}, \bar{D}$, $\left.\sigma_{l}, \sigma_{b}, \sigma_{D}, f_{*}, n\right)$, and each star has three-dimensional coordinates $(l, b, D)$. As we attempt to carry out the fit on small $5 \times 5 \mathrm{deg}^{2}$ patches on the sky around the assumed position prior $\left(l_{\text {prior }}, b_{\text {prior }}\right)$, we always have a sufficient number of overdensity and background RRab stars for the fit as shown in Figures 1-11. The overdensity itself is fit by a threedimensional Gaussian describing the spatial position of the overdensity $(\bar{l}, \bar{b}, \bar{D})$ and its extent $\left(\sigma_{l}, \sigma_{b}, \sigma_{D}\right)$. Thus a minimum number of two RRab stars within the overdensity, in addition to the background, is needed for a successful fit, albeit with large uncertainties when the number of RRab is small. All overdensities for which we later claim fitted positions and spatial extents meet these criteria, i.e., the minimum number of RRab stars is 3 .

\subsubsection{Model Priors}

We now lay out the "pertinent range," across which the priors for the model parameters $\boldsymbol{\theta}=\left(\bar{l}, \bar{b}, \bar{D}, \sigma_{l}, \sigma_{b}, \sigma_{D}, f_{*}, n\right)$ are given. Based on Tables 1 and 2 , we can set priors on $\bar{l}, \bar{b}, \bar{D}$. In general, we allow a rather wide prior, i.e., allow the on-sky positions to vary around the object's center by $\pm 5^{\circ}$, and the heliocentric mean distance $\bar{D}$ to vary by $\pm 20 \mathrm{kpc}$. In cases with a second overdensity nearby (i.e., NGC 5024, NGC 5053), we set more rigid priors on $\bar{l}, \bar{b}, \bar{D}$. As the halo profile tends to vary a lot on such small patches on the sky as are evaluated here, we allow the power-law index $n$ to vary between 1.7 and 5 .

Our complete prior function is then:

$$
\begin{aligned}
\ln p(\boldsymbol{\theta})= & \text { Uniform }\left(0.05 \leqslant f_{*}<1\right) \\
& + \text { Uniform }(1.7 \leqslant n<5.0) \\
& + \text { Uniform }\left(\log \left|\bar{l}-l_{\mathrm{tab}}\right|<\log \text { offset_deg }\right) \\
& + \text { Uniform }\left(\log \left|\bar{b}-b_{\mathrm{tab}}\right|<\log \text { offset_deg }\right) \\
& + \text { Uniform }\left(\log \left|\bar{D}-D_{\mathrm{tab}}\right|<\log \text { offset_kpc }\right) \\
& + \text { Uniform }\left(\log (0.1)<\log \left(\sigma_{l}\right)<\log (10)\right) \\
& + \text { Uniform }\left(\log (0.1)<\log \left(\sigma_{b}\right)<\log (10)\right) \\
& + \text { Uniform }\left(\log (0.1)<\log \left(\sigma_{D}\right)<\log (20)\right),
\end{aligned}
$$

where the index "tab" denotes the values from Tables 1 and 2 , respectively, and offset_deg $=5^{\circ}$, offset_kpc $=20 \mathrm{kpc}$ for all overdensities except for NGC 5024, NGC 5053. For NGC 5024 and NGC 5053, we set the offset to offset_deg $=1^{\circ}$.

\subsection{Fitting Tests on Mock Data}

In order to test the methodology for fitting the density as discussed in Section 4, we created mock data samples of RRab stars in the Galactic halo, superimposed Gaussian mock overdensities with typical distance, extent, and star count to mimic dwarf galaxies and globular clusters, and finally added noise in distance to mimic the distance uncertainty of $3 \%$. We then applied our fitting method.

Figure 13 shows two examples of a simulated distribution of halo and overdensity RRab along with a fit, analogous to the plots of the GC and dSph shown in Figures 1-11. One of them is an overdensity with a high contrast against the background of (mock) field stars that resembles a $\mathrm{dSph}$, whereas the other is a sparse overdensity, comparable to typical GC. We give their distribution in $(l, b, D)$ space as well as their marginalized distributions, the distribution these mock stars were drawn from and the best-fit distribution after applying our fitting methodology.

We find results that are consistent with the input model within reasonable uncertainties, which means that we are able to recover the input parameters for the models within their assumed parameter range.

\section{Results from Fitting}

When trying to fit all $\mathrm{dSph}$ from Table 1 and globular clusters from Table 2, we found that the data available for some of them do not allow for a reasonable fit. For the Crater II $\mathrm{dSph}$ at a distance of $\sim 120 \mathrm{kpc}$, the PS1 RRab catalog does not contain enough sources for a good fit, not surprising given the large distance. As Sagittarius dSph lies at the edge of the PS1 $3 \pi$ footprint, we cannot successfully fit its on-sky position $(l, b)$, but we can fit its heliocentric distance $D$. Among the globular clusters, we have to exclude NGC 4147, NGC 5634, NGC 5694, NGC 5897, IC 1257, NGC 6093 (M80), NGC 6171 (M107), NGC 6356, NGC 6402 (M14), NGC 6426, NGC 7099 (M30), Pal 1, and Pal 13, as in those cases, we find too few, if any, RRab stars associated with these overdensities picked up by the PS1 $3 \pi$ survey.

In Tables 3 and 4 as well as in Figures 1-11, we give the $\mathrm{dSph}$ and GC best-fit parameters we get from successfully carrying out the fitting as described in Section 4. Tables 3 and 4 list the name, fitted position $(l, b, D)$, fitted angular extent $\sigma_{l}$, $\sigma_{b}$, and depth $\sigma_{D}$ assuming a multivariate Gaussian, as well as the derived linear extents $(\Delta l, \Delta b, \Delta D)$ as

$$
\begin{gathered}
\Delta l=2 \sqrt{2\left((D \cos (b))^{2}\right)\left(1-\cos \left(\sigma_{l}\right)\right)} \\
\Delta b=2 D \tan \left(\sigma_{b}\right) \\
\Delta D=2 \sigma_{D} .
\end{gathered}
$$

We also give the axis ratios $\Delta D / \Delta l$ and $\Delta D / \Delta b$ describing the morphology, as well as the number of sources found in the $\mathrm{dSph}$ or globular cluster in each case.

\subsection{Comments on Individual Dwarf Galaxies and Globular Clusters}

We now comment on the fitting results for individual dwarf galaxies and globular clusters.

We fitted the five dwarf galaxies Sagittarius dSph, Sextans $\mathrm{dSph}$, Draco dSph, Ursa Minor Dwarf dE, and Ursa Major I dSph. 
Table 3

Fitted Dwarf Galaxies

\begin{tabular}{|c|c|c|c|c|c|c|c|c|}
\hline Name & $\begin{array}{c}\text { Fitted } \\
\left(l\left[^{\circ}\right], b\left[\left[^{\circ}\right], D[\mathrm{kpc}]\right)\right.\end{array}$ & $\begin{array}{c}\text { Fitted } \\
\left(\sigma_{l}\left[^{\circ}\right], \sigma_{b}\left[^{\circ}\right], \sigma_{D}[\mathrm{kpc}]\right)\end{array}$ & $\Delta l[\mathrm{kpc}]$ & $\Delta b[\mathrm{kpc}]$ & $\Delta D[\mathrm{kpc}]$ & $\Delta D / \Delta l$ & $\Delta D / \Delta b$ & Sources \\
\hline Draco dSph & $86.37_{-0.01}^{+0.01}, 34.71_{-0.01}^{+0.01}, 74.26_{-0.18}^{+0.18}$ & $0.13_{-0.01}^{+0.01}, 0.13_{-0.01}^{+0.01}, 2.40_{-0.15}^{+0.17}$ & 0.28 & 0.33 & 4.81 & 14.48 & 14.66 & 191 \\
\hline Sextans dSph & $243.55_{-0.04}^{+0.04}, 42.26_{-0.03}^{+0.04}, 81.42_{-0.40}^{+0.41}$ & $0.26_{-0.03}^{+0.03}, 0.23_{-0.03}^{+0.04}, 3.24_{-0.37}^{+0.43}$ & 0.55 & 0.66 & 6.49 & 9.83 & 9.87 & 99 \\
\hline Ursa Major I dSph & $159.38_{-1.48}^{+2.07}, 54.43_{-0.39}^{+2.79}, 94.33_{-4.94}^{+10.80}$ & $0.34_{-0.20}^{+2.53}, 0.33_{-0.19}^{+2.05}, 2.59_{-1.11}^{+5.90}$ & 0.65 & 1.07 & 5.18 & 4.84 & 4.84 & 4 \\
\hline Ursa Minor Dwarf dE4 & $105.00_{-0.06}^{+0.62}, 44.74_{-0.06}^{+0.36}, 68.41_{-0.51}^{+0.51}$ & $0.16_{-0.03}^{+0.86}, 0.14_{-0.02}^{+1.45}, 2.17_{-0.59}^{+3.10}$ & 0.22 & 0.33 & 4.35 & 19.77 & 13.11 & 53 \\
\hline
\end{tabular}

Note. The best-fit positions $(l, b, D)$ and extent $\left(\sigma_{l}, \sigma_{b}, \sigma_{D}\right)$ of dwarf galaxies from Table 1, along with their $1 \sigma$ uncertainties. Assuming an ellipsoidal shape for each dwarf galaxy, we calculate their axis ratios by first translating their angular extent $\left(\sigma_{l}, \sigma_{b}\right)$ into a linear extent $(\Delta l, \Delta b)$ as given by Equations (9) and (10), and then use the line-of-sight depth $\Delta D=2 \sigma_{D}$ (11) to calculate the axis ratios $\Delta D / \Delta l, \Delta D / \Delta b$. In this table, Sagittarius Dwarf dSph and Crater II dSph from Table 1 are missing, as we were not able to determine reasonable fits for them. For details on this, see Section 4.

Table 4

Fitted Globular Clusters

\begin{tabular}{|c|c|c|c|c|c|c|c|c|}
\hline Name & $\begin{array}{c}\text { Fitted } \\
\left(l\left[{ }^{\circ}\right], b\left[{ }^{\circ}\right], D[\mathrm{kpc}]\right)\end{array}$ & $\begin{array}{c}\text { Fitted } \\
\left(\sigma_{l}\left[^{\circ}\right], \sigma_{b}\left[^{\circ}\right], \sigma_{D}[\mathrm{kpc}]\right)\end{array}$ & $\Delta l[\mathrm{kpc}]$ & $\Delta b[\mathrm{kpc}]$ & $\Delta D[\mathrm{kpc}]$ & $\Delta D / \Delta l$ & $\Delta D / \Delta b$ & Sources \\
\hline NGC 2419 & $180.36_{-0.03}^{+0.03}, 25.24_{-0.03}^{+0.04}, 79.70_{-0.37}^{+0.32}$ & $0.11_{-0.01}^{+0.01}, 0.11_{-0.01}^{+0.02}, 4.24_{-1.14}^{+1.33}$ & 0.28 & 0.30 & 8.47 & 30.25 & 27.81 & 8 \\
\hline NGC 4590 (M68) & $299.62_{-0.08}^{+0.12}, 36.07_{-0.11}^{+0.10}, 10.48_{-0.28}^{+0.26}$ & $0.15_{-0.04}^{+0.68}, 0.15_{-0.04}^{+0.65}, 0.66_{-0.13}^{+1.29}$ & 0.04 & 0.05 & 1.32 & 33.0 & 24.15 & 5 \\
\hline NGC 5053 & $335.78_{-0.18}^{+0.19}, 78.93_{-0.14}^{+0.16}, 16.66_{-0.26}^{+0.28}$ & $0.30_{-0.16}^{+1.08}, 0.18_{-0.06}^{+1.10}, 0.75_{-0.20}^{+3.69}$ & 0.03 & 0.10 & 1.50 & 50.0 & 14.54 & 4 \\
\hline NGC 5272 (M3) & $42.20_{-0.07}^{+0.08}, 78.71_{-0.02}^{+0.02}, 10.48_{-0.07}^{+0.07}$ & $0.45_{-0.05}^{+0.05}, 0.10_{-0.002}^{+0.01}, 0.51_{-0.01}^{+0.02}$ & 0.03 & 0.04 & 1.02 & 34.0 & 27.05 & 56 \\
\hline NGC 6229 & $73.64_{-0.04}^{+0.04}, 40.31_{-0.04}^{+0.04}, 29.94_{-0.19}^{+0.17}$ & $0.11_{-0.01}^{+0.02}, 0.11_{-0.01}^{+0.03}, 0.61_{-0.08}^{+0.19}$ & 0.09 & 0.12 & 1.22 & 13.5 & 10.31 & 12 \\
\hline NGC 6864 (M75) & $20.28_{-0.23}^{+0.25},-25.76_{-0.28}^{+0.20}, 20.79_{-0.35}^{+0.32}$ & $0.25_{-0.13}^{+1.46}, 0.34_{-0.21}^{+2.70}, 1.59_{-0.93}^{+4.27}$ & 0.16 & 0.24 & 3.17 & 19.8 & 13.01 & 4 \\
\hline NGC 6934 & $52.12_{-0.04}^{+0.04},-18.87_{0.04}^{+0.03}, 16.77_{-0.21}^{+0.21}$ & $0.11_{-0.01}^{+0.03}, 0.11_{-0.01}^{+0.02}, 0.57_{-0.05}^{+0.16}$ & 0.06 & 0.07 & 1.14 & 19.00 & 17.14 & 10 \\
\hline NGC 6981 (M72) & $335.16_{-0.03}^{+0.03},-32.70_{-0.03}^{+0.03}, 17.51_{-0.17}^{+0.15}$ & $0.11_{-0.01}^{+0.01}, 0.11_{-0.01}^{+0.01}, 0.56_{-0.05}^{+0.11}$ & 0.06 & 0.07 & 1.12 & 18.70 & 16.90 & 15 \\
\hline NGC 7006 & $63.78_{-0.03}^{+0.03},-19.39_{-0.03}^{+0.03}, 40.12_{-0.15}^{+0.16}$ & $0.11_{-0.004}^{+0.01}, 0.11_{-0.01}^{+0.01}, 0.59_{-0.06}^{+0.11}$ & 0.15 & 0.15 & 1.17 & 7.80 & 7.87 & 16 \\
\hline NGC 7078 (M15) & $65.03_{-0.08}^{+0.11},-27.32_{-0.08}^{+0.07}, 11.07_{-0.22}^{+0.24}$ & $0.15_{-0.04}^{+1.07}, 0.13_{-0.03}^{+0.95}, 0.60_{-0.08}^{+2.69}$ & 0.05 & 0.05 & 1.21 & 24.20 & 23.42 & 10 \\
\hline
\end{tabular}

Note. The best-fit positions $(l, b, D)$ and extent $\left(\sigma_{l}, \sigma_{b}, \sigma_{D}\right)$ of globular clusters from Table 2, along with their $1 \sigma$ uncertainties. Assuming an ellipsoidal shape for each globular cluster, we calculate their axis ratios by first translating their angular extent $\left(\sigma_{l}, \sigma_{b}\right)$ into a linear extent $(\Delta l, \Delta b)$ as given by Equations (9) and (10), and then use the line-of-sight depth $\Delta D=2 \sigma_{D}(11)$ to calculate the axis ratios $\Delta D / \Delta l, \Delta D / \Delta b$. In this table, NGC 4147, NGC 5634, NGC 5694, NGC 5897, IC 1257, NGC 6093 (M80), NGC 6171 (M107), NGC 6284, NGC 6356, NGC 6402 (M14), NGC 6426, NGC 7099 (M30), Pal 1, and Pal 13 from Table 2 are missing, as we were not able to determine reasonable fits for them. For details on this, see Section 4.

As the Sagittarius dSph lies at the edge of the PS1 $3 \pi$ footprint, we cannot successfully fit its on-sky position $(l, b)$, but we can fit its heliocentric distance $D$. This can be clearly seen from Figure 2. For this reason, we do not give any values dependent on the fitted $(l, b)$ position in the tables.

For the other dwarf galaxies, we found that the fit is well defined as these overdensities typically have $\gtrsim 100$ sources, except for Ursa Major I. In the case of the latter, the marginalized best-fit model for $l$ as given in the lower left panel of Figure 3 does not seem to match the histogram well. However, this is only an effect due to the marginalization in the histogram, as it shows all sources independent of their distance, whereas the Gaussian is centered on the fitted $(l, b, D)$. Comparing the best-fit model to the map of stars as given in the upper left panel reveals the accuracy of the fit.

Regarding globular clusters, we have to deal with overdensities of fewer sources, given that the central regions are too crowded for the PS1 RRab catalog (Sesar et al. 2017).
Comparing, for example, the panel of NGC 5024 in Figure 12 to the histograms in Figure 5, we clearly see that stars are missing near the assumed center of the globular cluster. However, the fit is stable enough to identify a reasonable center position.

As for the dwarf galaxy Ursa Minor Dwarf dE, in the case of the globular clusters NGC 5024, NGC 5904, NGC 4590, NGC 6864, and NGC 7089, the marginalized best-fit model in $l$ and $b$ does not seem to match the histogram as well as might be expected, as the histogram shows all sources independent of their distance, while the Gaussian is centered on the fitted $(l, b$, $D$ ). Again, comparing the best-fit model to the map of stars, we see that the fit is reliable.

In some dSphs and GCs, only three to four RRab were detected. This is the case for Ursa Major I dSph, Ngc 5053, NGC 6864 (M75), NGC 8089 (M2), Pal 3, and Pal 5. In each of those cases, the overdensity is clearly visible in the map of RRab stars. The histograms might look unconvincing, as they 
show all sources independent of their distance and thus are heavily influenced by field stars. The corresponding best-fit model assigns a distance and position $(l, b, D)$ corresponding well with the map of RRab stars. However, we expect that the center, $(l, b, D)$, and the extent of the overdensity, $\left(\sigma_{l}, \sigma_{b}, \sigma_{D}\right)$ cannot be determined accurately from only three to four stars, as there is a high chance that the stars are not representative, i.e., are at the bright or shallow end or are not located symmetrically with respect to the center.

\subsection{Uncertainty-corrected Depth of Dwarf Galaxies and Globular Clusters}

In Tables 3 and 4 , we give the axis ratios $\Delta D / \Delta l$ and $\Delta D / \Delta b$ describing the morphology of each fitted overdensity. Starting with the dwarf galaxies, we find that all five of them are found to be quite elongated in the direction of $D$. In contrast, their values for $\Delta D / \Delta l$ and $\Delta D / \Delta b$ are comparable (except for Sagittarius $\mathrm{dSph}$, where we cannot calculate them), which is not surprising from Figures 1 and 3 showing a very round shape in $(l, b)$. We now check if the elongation in $D$ direction can be explained by the distance uncertainty.

For Sextans dSph, Draco dSph, Ursa Minor Dwarf dE4, and Ursa Major I dSph, we find a $\Delta D / \Delta l$ of $9.83,14.48,19.77$, and 4.84, respectively. Using Equation (5) with $\epsilon_{D}=0.03 D$, we calculate the uncertainty-corrected (true) line-of-sight depth $(\Delta D)^{\prime}=2 \sigma^{\prime}{ }_{D}=2 \sqrt{\left(\sigma_{D}^{2}-\epsilon_{D}^{2}\right)}=2 \sqrt{\left(\sigma_{D}^{2}-(0.03 D)^{2}\right)}$. We find thus $(\Delta D)^{\prime}=4.28$ for the Sextans dSph, $(\Delta D)^{\prime}=1.79$ for the Draco dSph, $(\Delta D)^{\prime}=1.41$ for the Ursa Minor Dwarf $\mathrm{dE} 4,(\Delta D)^{\prime}=0.94$ for the Ursa Major I dSph.

For the Ursa Major I dSph, $\epsilon_{D}$ must be $\lesssim 0.027 D$ instead of $0.03 D$ to make the result sensible. With the values for $\Delta l$ given in Table 3 and $\epsilon_{D}=0.03 D$, we calculate $\Delta D / \Delta l=7.78$ for Sextans dSph, 6.36 for Draco dSph, 6.40 for Ursa Minor Dwarf $\mathrm{dE} 4$, and 1.44 for Ursa Major I dSph.

This means that introducing a distance uncertainty, which is appropriate as shown by Sesar et al. (2017), reduces the elongation in the $D$ direction, but is still far away from an axis ratio of 1 . As the distance uncertainty $\epsilon_{D}$ and the true line-ofsight extent $\sigma_{D}^{\prime}$ add in quadrature to make the fitted line-ofsight extent $\sigma_{D}$, a slight variation in $\epsilon_{D}$ from the nominal $3 \%$ derived by Sesar et al. (2017) would be able to explain all of the asymmetry. For example, in the case of for the Sextans $\mathrm{dSph}, \epsilon_{D}=0.0396 D$ instead of $0.030 D$ would result into a $(\Delta D)^{\prime}=0.55$ and thus $(\Delta D)^{\prime} / \Delta l \sim 1$. Similarly, an axis ratio of 1 could be achieved for the Draco dSph with $\epsilon_{D}=0.0322 D$, for the Ursa Minor Dwarf dE4 with $\epsilon_{D}=0.0316 D$, and for the Ursa Major I dSph with $\epsilon_{D}=0.027 D$.

In addition to variations in the distance uncertainties, for globular clusters we suggest that fitting uncertainties and especially shot noise are responsible for the non-spherical axis ratios we find. This means, because of the expected small lineof-sight extent, even with a distance uncertainty of $3 \%$ we cannot say much about the line-of-sight extent for GC and dwarf galaxies at the distances considered here.

\subsection{The Radii of Globular Clusters and Dwarf Galaxies}

We also compared our fitted extent $\sigma_{l}, \sigma_{b}$ to the tidal radius and core radius.

For globular clusters, we use the tidal radius $r_{t}$ and core radius $r_{c}$ from the 2010 update of Harris (1996). We use the core radii from Stoehr et al. (2002) for the dwarf galaxies Draco
dSph, Sextans dSph and Ursa Minor Dwarf dE4. A core radius for Ursa Major I dSph is provided by Simon \& Geha (2007). We use tidal radii from Odenkirchen et al. (2001; Draco dSph), Roderick et al. (2016; Sextans dSph), and Kleyna et al. (1998; Ursa Minor Dwarf dE4). A tidal radius for Ursa Major I dSph is not available.

Tables 7 and 8 and Figure 14 summarize these comparisons.

We find that for globular clusters, while the distribution shows a lot of scatter, our estimated extent from $\max \left(\sigma_{l}, \sigma_{b}\right)$ represents significant fractions of the tidal radius. We find sources far beyond the core radius. For the dwarf galaxies in our sample, except Ursa Major I dSph, our estimated extent from $\max \left(\sigma_{l}, \sigma_{b}\right)$ matches the core radius quite well. In all cases, our estimated extent is below the tidal radius. We do not pick up sources within or near the core radius for GC. We attribute this to the fact that in the case of GC, we are losing the RRab stars in the cores, as mentioned in Section 3.

\section{Mean Distances to Other Globular Clusters and Dwarf Galaxies}

For those dwarf galaxies and globular clusters from Tables 1 and 2, which do not have enough sources to carry out the fitting process described in Section 4, we derived their mean distances (in the case of finding multiple RRab stars likely associated with the overdensity) or give the distance to the single RRab star found within this overdensity.

Tables 5 and 6 list the distances, along with the number of RRab stars found in each case.

In total, for each of the $13 \mathrm{GC}$ and $6 \mathrm{dSph}$ we found at least one RRab star associated with the overdensity. For those with at least two RRab stars, we give the mean distance, and otherwise, the distance from the only RRab star found. In most cases, our distance estimate lies well within the $3 \%$ uncertainty we claim for the PS1 $3 \pi$ sample of RR Lyrae stars.

For some overdensities, we find a lot of stars in the field at about the same distance. This is the case for NGC 5897, where one RRab star is very close to the assumed coordinates of this GC, but there are in total up to four stars that could be associated with that GC. For NGC 6402 (M14), the field is even more crowded; there are many sources in the field at that distance without revealing an overdensity.

In the case of NGC 6356, we find one source close to the coordinates given for this GC, but at a different distance: the RRab star from our catalog is at a heliocentric distance of $11.02 \mathrm{kpc}$, whereas Harris (1996) gives a heliocentric distance of $15.1 \mathrm{kpc}$ for NGC 6356. In the case of Pal 1, we find no RRab stars clearly associated with this GC. For Pal 13, we find three RRab stars for which we calculate a mean distance of $23.59 \mathrm{kpc}$, which is about $2.5 \mathrm{kpc}$ lower than the distance given by the recent compilation of Harris (1996). Siegel et al. (2010) claim a distance of $24.8 \mathrm{kpc}$, which is within our distance precision. The same is the case for the dwarf galaxy Bootes I dSph. We find a mean heliocentric distance of $60.61 \mathrm{kpc}$, which is about $5 \mathrm{kpc}$ off from the distance given by Okamoto et al. (2012). However, our distance estimate matches very well the distance of $60.4 \mathrm{kpc}$ by Hammer et al. (2018). For Segue $1 \mathrm{dSph}$, it is difficult to identify which sources are likely associated with this dwarf galaxy, as there are many sources in the field at about that distance. For the farthest dwarf galaxy in our sample, Crater II dSph, our heliocentric distance estimate of $105.48 \mathrm{kpc}$ is somewhat lower 
Table 5

Dwarf Galaxies with Too Few RRab Stars

\begin{tabular}{lrcc}
\hline \hline Name & Mean & Sources & Comment \\
& $\bar{D}[\mathrm{kpc}]$ & & \\
\hline Aquarius II dSph & 107.87 & 1 & \\
Bootes I dSph & 60.61 & 2 & \\
Crater II dSph & 105.48 & 2 & \\
Segue 1 dSph & 23.24 & 1 & up to two additional sources \\
& & & nearby \\
Segue 2 dSph & 33.31 & 1 & \\
Ursa Major II & 33.02 & 1 & \\
$\quad$ & & & \\
Dwarf & & &
\end{tabular}

Note. The mean positions $(l, b, D)$ of those dwarf galaxies from Table 1 that do not have enough sources in the PS1 catalog of RR Lyrae stars for carrying out the fitting process described in Section 4.

than distance estimates in the literature, i.e., the $112 \mathrm{kpc}$ claimed by Joo et al. (2018).

\section{PL Relations}

In one of our previous papers (Sesar et al. 2017), we used the period-absolute magnitude-metallicity (PLZ) relation known for RR Lyrae stars to calculate the RRab star's distances we use in the paper at hand. The PLZ relation is given as (e.g., Catelan et al. 2004; Sollima et al. 2006):

$$
\begin{aligned}
M_{\lambda}= & \alpha_{\lambda} \log _{10}\left(P / P_{\mathrm{ref}}\right)+\beta_{\lambda}\left([\mathrm{Fe} / \mathrm{H}]-[\mathrm{Fe} / \mathrm{H}]_{\mathrm{ref}}\right) \\
& +M_{\mathrm{ref}, \lambda}+\epsilon,
\end{aligned}
$$

where $\lambda$ denotes the bandpass, $P$ is the period of pulsation, $M_{\text {ref }}$ is the absolute magnitude at some reference period and metallicity (here chosen to be $P_{\text {ref }}=0.6$ days, $[\mathrm{Fe} / \mathrm{H}]_{\text {ref }}=-1.5 \mathrm{dex}$ ), and $\alpha$, $\beta$ describe the dependence of the absolute magnitude on period and metallicity. The $\epsilon$ is a standard normal random variable centered on 0 and with a standard deviation of the uncertainty in $M_{\lambda}$ in order to model the intrinsic scatter in the absolute magnitude convolved with unaccounted measurement uncertainties.

As PS1 $3 \pi$ itself has no metallicity information available, we constrained the PLZ relation Equation (12) in PS1 bandpasses using metallicities and distance moduli of PS1 RRab stars in the five Galactic globular clusters NGC 6171, NGC 5904, NGC 4590, NGC 6341, and NGC 7078 (Sesar et al. 2017). Table 1 in Sesar et al. (2017) gives the fitted PLZ relations in all bandpasses. The resulting relation in the $i_{\mathrm{P} 1}$ band is then used to constrain distances also for stars where no metallicity is available.

In the case of no available metallicity, the expression for the absolute magnitude in the $i_{\mathrm{P} 1}$ band, $M_{i_{\mathrm{P} 1}}$, simplifies to (Sesar et al. 2017, Equation (5)): $M_{i_{\mathrm{P} 1}}=-1.77 \log _{10}(P / 0.6)+0.46$, and, in general, the expression for the absolute magnitude in the $\lambda_{\mathrm{P} 1}$ band simplifies to

$$
M_{\lambda_{\mathrm{P} 1}}=\alpha_{\lambda} \log _{10}(P / 0.6)+M_{\mathrm{ref}, \lambda} .
$$

Distances are then constrained using the dereddened fluxaveraged $i_{\mathrm{P} 1}$-band magnitude (this is $i_{\mathrm{F}}$ in Sesar et al. 2017) and Equation (13). This equation was used to calculate the PS1 $3 \pi$ RRab distances we use in this paper.

Now, after having fitted the spatial extent and distance of 16 globular clusters, we are interested in comparing the fitted PLZ relation from Sesar et al. (2017) to fits carried out for each cluster using the $[\mathrm{Fe} / \mathrm{H}]$ from spectra of red giants of Carretta et al. (2009; Table A1). For each RRab star in each GC, we
Table 6

Globular Clusters with Too Few RRab Stars

\begin{tabular}{lccc}
\hline \hline Name & Mean & Sources & Comment \\
& $\bar{D}[\mathrm{kpc}]$ & & \\
\hline IC 1257 & 27.24 & 1 & \\
NGC 4147 & 18.54 & 1 & \\
NGC 5634 & 25.81 & 1 & \\
NGC 5694 & 33.96 & 1 & up to three additional sources \\
NGC 5897 & 12.91 & 1 & nearby \\
NGC 6093 & 10.74 & 2 & many field stars at this distance \\
NGC 6171 (M107) & 6.01 & 7 & \\
NGC 6356 & 11.02 & 1 & \\
NGC 6402 (M14) & & & nothing detected at this position: \\
NGC 6426 & 19.83 & 5 & mean $D=10.08$ kpc \\
NGC 7099 (M30) & 8.41 & 2 & \\
Pal 1 & & & \\
Pal 13 & 23.59 & 3 &
\end{tabular}

Note. The mean positions $(l, b, D)$ of those globular clusters from Table 2 that do not have enough sources in the PS1 catalog of RR Lyrae stars for carrying out the fitting process described in Section 4.

have the period from the RRab catalog, the $[\mathrm{Fe} / \mathrm{H}]$ - assumed to be constant for a given GC-from Carretta et al. (2009) and the fitted distance modulus (DM) and distance from the RRab catalog.

For Figure 15, we selected the RRab stars for each globular cluster and plot their dereddended apparent $r$-band magnitude $\left(r_{F}\right.$ in the PS1 RRab catalog) versus their period. The typical trend of a PL relation is clearly visible.

We then took a closer look at the individual globular clusters. For each of them, in each bandpass $\lambda \in\left\{g_{\mathrm{P} 1}, \ldots, z_{\mathrm{P} 1}\right\}$, we plot each star's dereddened apparent magnitude $m_{\lambda}$ versus $P$. We also plot the apparent (unreddened) magnitude as expected from the PLZ relation $\alpha_{\lambda} \log _{10}\left(P / P_{\text {ref }}\right)+$ $\beta_{\lambda}\left([\mathrm{Fe} / \mathrm{H}]-[\mathrm{Fe} / \mathrm{H}]_{\text {ref }}\right)+M_{\text {ref }}+\mathrm{DM}$ versus $P$, as well as the apparent magnitude as expected from the equation without metallicity $\alpha_{\lambda} \log _{10}\left(P / P_{\text {ref }}\right)+M_{\text {ref }}+$ DM versus $P$.

This results in Figures 16-31. The error bars in the predicted apparent magnitudes correspond to an uncertainty of $\sim 0.06 \mathrm{mag}$ in the absolute magnitude and thus DM (Sesar et al. 2017). As the DM was derived from the $i_{\mathrm{P} 1}$ band, in this band, per definition, the observed magnitude and the apparent magnitude as predicted without $[\mathrm{Fe} / \mathrm{H}]$ are the same. We find that for most of the 16 globular clusters we have evaluated, the predicted apparent magnitude with $[\mathrm{Fe} / \mathrm{H}]$ (blue markers in the figures) is a bit brighter than the predicted apparent magnitude without taking into account $[\mathrm{Fe} / \mathrm{H}]$ (black markers), and this is again a bit brighter than the observed dereddened apparent magnitude (orange markers). NGC 6864 (Figure 24) is the only GC where the predicted apparent magnitude with $[\mathrm{Fe} / \mathrm{H}]$ is higher than the one predicted without $[\mathrm{Fe} / \mathrm{H}]$. It has the highest metallicity in the sample of GC studied here.

The offset in the predicted apparent magnitude depends on the metallicity of the GC in this case. The predicted apparent magnitudes with $[\mathrm{Fe} / \mathrm{H}]$ (blue markers in the figures) are calculated using Equation (12) and the DM for each RRab star given in the PS1 RRab catalog (Sesar et al. 2017). For the black markers, we neglect the term $\beta_{\lambda}\left([\mathrm{Fe} / \mathrm{H}]-[\mathrm{Fe} / \mathrm{H}]_{\mathrm{ref}}\right)$ from Equation (12), and again use the DM. As $\beta_{\lambda}$ ranges from 0.06 to $0.09,[\mathrm{Fe} / \mathrm{H}]_{\text {ref }}=-1.5$, and $[\mathrm{Fe} / \mathrm{H}]$, which ranges 
Table 7

Radii of Dwarf Galaxies

\begin{tabular}{lrrr}
\hline \hline Name & \multicolumn{1}{c}{$r_{t}$ [arcmin] } & $r_{c}$ [arcmin] & max $\left(\sigma_{l}, \sigma_{b}\right)$ [arcmin] \\
\hline Draco dSph & $40.1 \pm 0.9$ (Odenkirchen et al. 2001) & 8.33 (Stoehr et al. 2002) & 7.8 \\
Sextans dSph & $83.2 \pm 7.1$ (Roderick et al. 2016) & 14.14 (Stoehr et al. 2002) & 16.5 \\
Ursa Minor Dwarf dE4 & $34 \pm 2.4$ (Kleyna et al. 1998) & 10.5 (Stoehr et al. 2002) & 9.6 \\
Ursa Major I dSph & & 7.28 (Simon \& Geha 2007) & 20.4 \\
\hline
\end{tabular}

Note. Comparison of tidal radii $r_{t}$ and core radii $r_{c}$ to the angular extent $\max \left(\sigma_{l}, \sigma_{b}\right)$ from our analysis for the four fitted dwarf galaxies. Ursa Major I dSph lacks a published tidal radius. We give uncertainties as far as provided. See also Figure 14.

Table 8

Radii of Globular Clusters

\begin{tabular}{lrcc}
\hline \hline Name & $r_{t}$ [arcmin] & $r_{c}[\operatorname{arcmin}]$ & $\max \left(\sigma_{l}, \sigma_{b}\right)$ [arcmin] \\
\hline NGC 2419 & 8.74 & 0.35 & 6.6 \\
NGC 4590 (M68) & 30.34 & 0.69 & 9.0 \\
NGC 5024 (M53) & 21.75 & 0.36 & 21 \\
NGC 5053 & 13.67 & 1.98 & 18 \\
NGC 5272 (M3) & 38.19 & 0.55 & 27 \\
NGC 5466 & 34.24 & 1.64 & 7.2 \\
NGC 5904 (M5) & 28.40 & 0.42 & 7.8 \\
NGC 6229 & 5.38 & 0.13 & 6.6 \\
NGC 6864 (M75) & 7.28 & 0.10 & 6.4 \\
NGC 6934 & 8.37 & 0.25 & 6.6 \\
NGC 6981 (M72) & 9.15 & 0.54 & 6.6 \\
NGC 7006 & 6.34 & 0.24 & 9.0 \\
NGC 7078 (M15) & 21.50 & 0.07 & 37.2 \\
NGC 7089 (M2) & 21.45 & 0.34 & 20.4 \\
Pal 3 & 4.81 & 0.48 & 34.8 \\
Pal 5 & 16.28 & 3.25 & \\
\hline
\end{tabular}

Note. Tidal radius $r_{t}$, core radius $r_{c}$ from the 2010 version of Harris (1996), as well as the angular extent $\max \left(\sigma_{l}, \sigma_{b}\right)$ from our analysis for all the fitted globular clusters. See also Figure 14.

from -2.33 to -1.29 , is $<-1.5$ for most $\mathrm{GC}$, this term is negative. Thus, for most of the 16 globular clusters we have evaluated, the predicted apparent magnitude with $[\mathrm{Fe} / \mathrm{H}]$ (blue markers in the figures) is a bit brighter than the predicted apparent magnitude without taking into account $[\mathrm{Fe} / \mathrm{H}]$ (black markers).

The offset between observed and predicted apparent magnitudes can be explained by the way the distance to the RRab stars was derived. The DM we use here to calculate the predicted magnitudes was derived in Sesar et al. (2017) using the flux-averaged $i_{\mathrm{P} 1}$-band magnitude. Thus, for the $i$ band, the observed magnitude (orange markers) and the magnitude predicted without $[\mathrm{Fe} / \mathrm{H}]$ agree, while for the other bands, we find the predicted magnitudes to deviate slightly from the observed ones (black versus orange markers). This deviation is on the order of the uncertainty claimed for the DM, 0.06 (rnd) \pm 0.03 (sys) mag (Sesar et al. 2017).

\section{Gaia DR2 Distances}

In this section, we compare the PS $13 \pi$ distances to those published for the second Gaia data release (hereafter Gaia DR2; Gaia Collaboration 2018).

According to Bailer-Jones et al. (2018), for the vast majority of stars in Gaia DR2, reliable distances cannot be obtained by inverting the parallax, but should be derived by an algorithm that accounts for the nonlinearity of the transformation. Carrying out a probabilistic approach, BailerJones et al. (2018) published distances to 1.33 billion stars in Gaia DR2.

To compare our heliocentric distance estimates $D_{\mathrm{PS} 1}$ from Sesar et al. (2017) to those of Gaia DR2 Bailer-Jones et al. (2018), we cross-matched our PS1 RRab sample of 44,403 stars with Gaia DR2, and found a cross-match for 43,791 sources. In Figure 32, we show the $D_{\text {GaiaDR2 }}$ and a rough distance estimate $1 /$ parallax versus $D_{\mathrm{PS} 1}$ for the RRab stars within $D_{\mathrm{PS} 1}<20 \mathrm{kpc}$.

At the present time, we find a rather large scatter in the distances, much larger than the PS1 distance uncertainty of $3 \%$ or an estimate of the distance uncertainty from the parallax error. When the parallax error exceeds $10 \%$, which happens at a heliocentric distance of about $5 \mathrm{kpc}$, the distances from Gaia DR2 become very unreliable. Bailer-Jones et al. (2018) already claim quite large uncertainties of their distance estimates. In Figure 33, we plot the upper and lower $68 \%$ confidence interval boundaries of their distance estimates for our cross-matched sample of RRab stars. According to Bailer-Jones et al. (2018), the confidence intervals are asymmetric with respect to the distance estimate $D_{\text {GaiaDR2 }}$ as a consequence of the nonlinear transformation from parallax to distance.

Up to now, the precision of those distances-which are calculated only from geometric principles without any assumptions on astrophysics such as pulsation or PLZ relationships - is not competitive with the precision of our distances from Sesar et al. (2017), and especially cannot be used for the distant GC and dwarfs. However, this might change at least partially for the end-of-mission data. Until then, we strongly recommend using the RRab distances from Sesar et al. (2017) and subsequent analysis instead of those from Gaia DR2.

\section{Discussion and Summary}

We started our analysis based on a list of dwarf galaxies within $3 \mathrm{Mpc}$ by McConnachie (2012) and a list of currently known globular clusters from a current online version of the Harris (1996) database. We excluded those outside the PS1 $3 \pi$ footprint and attempted to fit the $(l, b, D)$ distribution of the remaining ones. For the ones for which a fit was not possible due to only a small number of RRab stars available, we instead selected those stars and give their mean distance.

In Tables 9 and 10, we compare our distances for dwarf galaxies and GC to literature distances. For almost all dwarf galaxies and globular clusters, the estimated distances compared to those in the recent literature are well within our distance precision of $3 \%$. However, there are a few dwarf galaxies and 
Table 9

Distance Comparison for Dwarf Galaxies

\begin{tabular}{|c|c|c|c|c|}
\hline Name & $D[\mathrm{kpc}]$ & Method & Literature $D[\mathrm{kpc}]$ & Method and Reference \\
\hline Aquarius II dSph & 107.87 & 1 RRab & $107.900 \pm 3.300$ & BHB stars (Torrealba et al. 2016b) \\
\hline \multirow[t]{2}{*}{ Bootes I dSph } & 60.61 & mean of $2 \mathrm{RRab}$ & 65.3 & (BHB + RR Lyr) HSC/Subaru imaging (Okamoto et al. 2012) \\
\hline & & & $62 \pm 4$ & 15 RR Lyr (Siegel 2006) \\
\hline \multirow{3}{*}{ Crater II dSph } & 105.48 & mean of $2 \mathrm{RRab}$ & $\begin{array}{l}60.4 \\
\sim 120\end{array}$ & $\begin{array}{l}\text { (Hammer et al. 2018) } \\
\text { CMD (Torrealba et al. 2016a) }\end{array}$ \\
\hline & & & $112 \pm 5$ & RR Lyr (Joo et al. 2018) \\
\hline & & & $117.5 \pm 5$ & (McConnachie 2012) \\
\hline \multirow[t]{2}{*}{ Draco dSph } & $74.26_{-0.18}^{+0.18}$ & fit & $71 \pm 7$ & SDSS photometry (Odenkirchen et al. 2001) \\
\hline & & & $75.8 \pm 5.4$ & 94 RR Lyr (Bonanos et al. 2004) \\
\hline Sagittarius dSph & $28.18_{-0.10}^{+0.10}$ & fit & $26.3 \pm 1.8$ & RGB tip (Monaco et al. 2004) \\
\hline Segue $1 \mathrm{dSph}$ & 23.24 & $1 \mathrm{RRab}$ & $23 \pm 2$ & CMD fitting, esp. horizontal branch (Belokurov et al. 2007) \\
\hline Segue $2 \mathrm{dSph}$ & 33.31 & $1 \mathrm{RRab}$ & 35 & from 4 BHB stars (Belokurov et al. 2009) \\
\hline Sextans dSph & $81.42_{-0.40}^{+0.41}$ & fit & $84.2 \pm 3.3$ & RR Lyr from Dark Energy Camera imaging (Medina et al. 2018) \\
\hline \multirow[t]{2}{*}{ Ursa Minor Dwarf dE4 } & $68.41_{-0.51}^{+0.51}$ & fit & 76 & RR Lyr and HB (Bellazzini et al. 2002) \\
\hline & & & $76 \pm 4$ & distance from HB, considering metallicity of UMi (Carrera et al. 2002) \\
\hline \multirow[t]{2}{*}{ Ursa Major I dSph } & $94.33_{-4.94}^{+10.80}$ & fit & $96.8 \pm 4$ & V mag of HB, HSC/Subaru imaging study (Okamoto et al. 2008) \\
\hline & & & $97.3_{-5.7}^{+6.0}$ & variable stars in UMa 1 (Garofalo et al. 2013) \\
\hline Ursa Major II Dwarf & 33.02 & $1 \mathrm{RRab}$ & $34.7_{-1.9}^{+2.0}$ & 1 RR Lyr (Dall'Ora et al. 2012) \\
\hline
\end{tabular}

Note. Comparison of our distance estimates (column $D[\mathrm{kpc}]$ ) to reference values from literature. For our distance estimates, we note whether they are a result of the fitting process or are determined as a mean distance from a small number of RRab stars. For literature values, we give the method used to obtain the distance and the reference.

Table 10

Distance Comparison for Globular Cluster

\begin{tabular}{llll}
\hline \hline Name & $D[\mathrm{kpc}]$ & Method & Literature $D$ [kpc] \\
\hline IC 1257 & 27.24 & 1 RRab & 25 \\
NGC 2419 & $79.70_{-0.37}^{+0.32}$ & fit & 82.6 \\
NGC 4147 & 18.54 & 1 RRab & 19.3 \\
NGC 4590 (M68) & $10.48_{-0.28}^{+0.26}$ & fit & 10.3 \\
NGC 5024 (M53) & $18.25_{-0.13}^{+0.13}$ & fit & 17.9 \\
NGC 5053 & $16.66_{-0.26}^{+0.28}$ & fit & 17.4 \\
NGC 5272 (M3) & $10.48_{-0.07}^{+0.07}$ & fit & 10.2 \\
NGC 5466 & $15.76_{-0.14}^{+0.14}$ & fit & 16 \\
NGC 5634 & 25.81 & 1 RRab & 25.2 \\
NGC 5694 & 33.96 & 1 RRab & 35 \\
NGC 5897 & 12.91 & 1 RRab & 12.5 \\
NGC 5904 (M5) & $7.87_{-0.19}^{+0.19}$ & fit & 7.5 \\
NGC 6093 (M80) & 10.74 & 2 RRab & 10 \\
NGC 6171 (M107) & 6.01 & 7 RRab & 5.4 \\
NGC 6229 & $29.94_{-0.19}^{+0.17}$ & fit & 30.5 \\
NGC 6356 & 11.02 & 1 RRab & 15.1 \\
NGC 6426 & 19.83 & 5 RRab & 20.6 \\
NGC 6864 (M75) & $20.79_{-0.35}^{+0.32}$ & fit & 20.9 \\
NGC 6934 & $16.77_{-0.21}^{+0.21}$ & fit & 15.6 \\
NGC 6981 (M72) & $17.51_{-0.17}^{+0.15}$ & fit & 17 \\
NGC 7006 & $40.12_{-0.15}^{+0.16}$ & fit & 41.2 \\
NGC 7078 (M15) & $11.07_{-0.24}^{+0.24}$ & fit & 10.4 \\
NGC 7089 (M2) & $12.11_{-0.28}^{+0.39}$ & fit & 11.5 \\
NGC 7099 (M30) & 8.41 & 2 RRab & 8.1 \\
Pal 3 & $85.05_{-0.34}^{+0.32}$ & fit & 92.5 \\
Pal 5 & $21.66_{-0.30}^{+0.33}$ & fit & 23.2 \\
Pal 13 & 23.59 & 3 RRab & 26 \\
\hline
\end{tabular}

Note. Comparison of our distance estimates (column $D[\mathrm{kpc}]$ ) to reference values from the literature. For our distance estimates, we note whether they are a result of the fitting process or are determined as a mean distance from a small number of RRab stars. For literature values, we use the 2010 online version of the Harris (1996) catalog. globular clusters for which our distances deviate significantly from those given in the literature.

In the case of NGC 6356, we find only one source close to the coordinates given for this GC, but at a different distance: while the RRab star from our catalog is at a heliocentric distance of $11.02 \mathrm{kpc}$, the updated catalog of Harris (1996) gives a heliocentric distance of $15.1 \mathrm{kpc}$ for NGC 6356. For Pal 3 , we find three stars within a small distance range, resulting in a heliocentric distance estimate of $85.05 \mathrm{kpc}$, whereas Harris (1996) gives a distance of $92.5 \mathrm{kpc}$. For Pal 13, we find three RRab stars resulting in a mean distance of $23.59 \mathrm{kpc}$, which is about $2.5 \mathrm{kpc}$ off from the distance given by Harris (1996). However, this star might be a member of Pal 13, as Siegel et al. (2010) claim a distance of $24.8 \mathrm{kpc}$, which is within our distance precision. For IC 1257, we have found only one RRab star associated with this GC and estimated its distance to $27.24 \mathrm{kpc}$, in comparison to the $25 \mathrm{kpc}$ given by Harris (1996).

For the dwarf galaxy Bootes I dSph, we find a mean heliocentric distance of $60.61 \mathrm{kpc}$, which is about $5 \mathrm{kpc}$ off from the distance given by Okamoto et al. (2012) and about $1.5 \mathrm{kpc}$ off from the distance given by Siegel (2006). However, our distance estimate matches very well the distance of $60.4 \mathrm{kpc}$ given by Hammer et al. (2018). The most distant dwarf galaxy in our sample is Crater II dSph. Our heliocentric distance estimate of $105.48 \mathrm{kpc}$ for this dwarf galaxy is somewhat off from the $117.5 \mathrm{kpc}$ distance estimate reported by McConnachie (2012), but there are closer distance estimates like the $112 \pm 5 \mathrm{kpc}$ claimed by Joo et al. (2018), which agrees within the uncertainties with our result. For Sagittarius dSph, our distance estimate of $28.18 \mathrm{kpc}$ is slightly larger than, but within the uncertainties of the $26.3 \pm 1.8 \mathrm{kpc}$ result obtained by Monaco et al. (2004). For Ursa Minor Dwarf dE4, we find a distance of $68.41 \mathrm{kpc}$, whereas the distance is given as $76 \mathrm{kpc}$ from Bellazzini et al. (2002) as well as Carrera et al. (2002).

In some cases, it is hard to find stars associated with the overdensities, especially if there are many field stars within the 
assumed distance range of the dwarf galaxy or GC, and there is no apparent overdensity. This is the case for NGC 5897, where one RRab star is very close to the assumed coordinates of this $\mathrm{GC}$, but there are in total up to four stars that could be associated with that GC. For the GC NGC 6402 (M14), the field is even more crowded, so many sources in the field are at its assumed distance without revealing an overdensity. For NGC 6356, we find one source close to the coordinates given for this GC, but at a different distance: the RRab star from our catalog is at a heliocentric distance of $11.02 \mathrm{kpc}$, whereas Harris (1996) give a heliocentric distance of $15.1 \mathrm{kpc}$ for NGC 6356. In the case of Pal 1, we find no stars clearly associated with this GC. For Segue $1 \mathrm{dSph}$, it is difficult to identify the sources that might be associated with this dwarf galaxy, as there are many sources in the field at about that distance.

For the GC, even though these sources are closer, in most cases our distance uncertainties are larger than for dwarf galaxies. This probably occurs because we are missing sources near the centers of the GCs as the spatial density of sources is higher than that which can be handled by the PS1 analysis codes.

In this paper we have computed the extents $\Delta l, \Delta b, \Delta D$ and the axis ratios $\Delta D / \Delta l$ and $\Delta D / \Delta l$ for all dwarf galaxies and GCs in which a sufficient number of RRab were picked up in the PS1 RR Lyr database of Sesar et al. (2017). Our distances to the stellar overdensities we study here, including both GCs and dwarf galaxy satellites of the Milky Way, are accurate to better than 3\% when more than eight RRab occur within a system (i.e., a $\mathrm{GC}$ or a $\mathrm{dSph}$ ).

In the past 5 years many groups have attempted to determine distances to some of the dwarf galaxies using RRab, but they have each used their own procedures and calibrations to convert a mean RRab magnitude into a distance (see Tables 9 and 10 for references). Our work is unique in that every object, within the large sample we study, is treated identically and comes from the same survey, including the metallicity dependence of the RR luminosity, so that the distances for our large sample of objects are on the same scale across the entire part of the sky covered by the PS1 $3 \pi$ survey, for all halo stellar overdensities within which a sufficient number of RRab could be detected. Thus overall we believe that our distances for the sample of stellar overdensities in the Milky Way halo, i.e., globular clusters and dwarf galaxies, that we study here are more precise and more homogeneous than those in the published literature.

H.-W.R. acknowledges funding from the European Research Council under the European Unions Seventh Framework Programme (FP 7) ERC grant Agreement No. [321035].

The Pan-STARRS1 Surveys (PS1) have been made possible through contributions of the Institute for Astronomy, the University of Hawaii, the Pan-STARRS Project Office, the Max-Planck Society and its participating institutes, the Max Planck Institute for Astronomy, Heidelberg and the Max Planck Institute for Extraterrestrial Physics, Garching, The Johns Hopkins University, Durham University, the University of Edinburgh, Queen's University Belfast, the HarvardSmithsonian Center for Astrophysics, the Las Cumbres Observatory Global Telescope Network Incorporated, the National Central University of Taiwan, the Space Telescope Science Institute, the National Aeronautics and Space Administration under grant No. NNX08AR22G issued through the Planetary Science Division of the NASA Science Mission Directorate, the National Science Foundation under grant No. AST-1238877, the University of Maryland, and Eotvos Lorand University (ELTE) and the Los Alamos National Laboratory.

\section{Appendix}

Figures 1-11 give plots of the dwarf spheroidals and globular clusters including their $(l, b, D)$ fit.

Figure 12 give the incompleteness of our RRab sample towards the central regions of globular clusters.

Figure 13 gives a fit to two mock overdensities.

Figure 14 gives a comparison between the estimated extent of the overdensities in the present and the tidal radii radii from the literature.

Figure 15 shows the typical trend of a PL relation we get from dereddened $r$-band magnitudes and periods of the globular clusters.

Figures 16-31 give observed and predicted apparent magnitudes for the globular clusters, and PL relations constructed from that.

Figure 32 compares compare our RRab distance estimates to those of Gaia DR2.

Figure 33 shows the confidence interval boundaries on the Gaia distances of RRab stars. 


\section{Draco dSph}
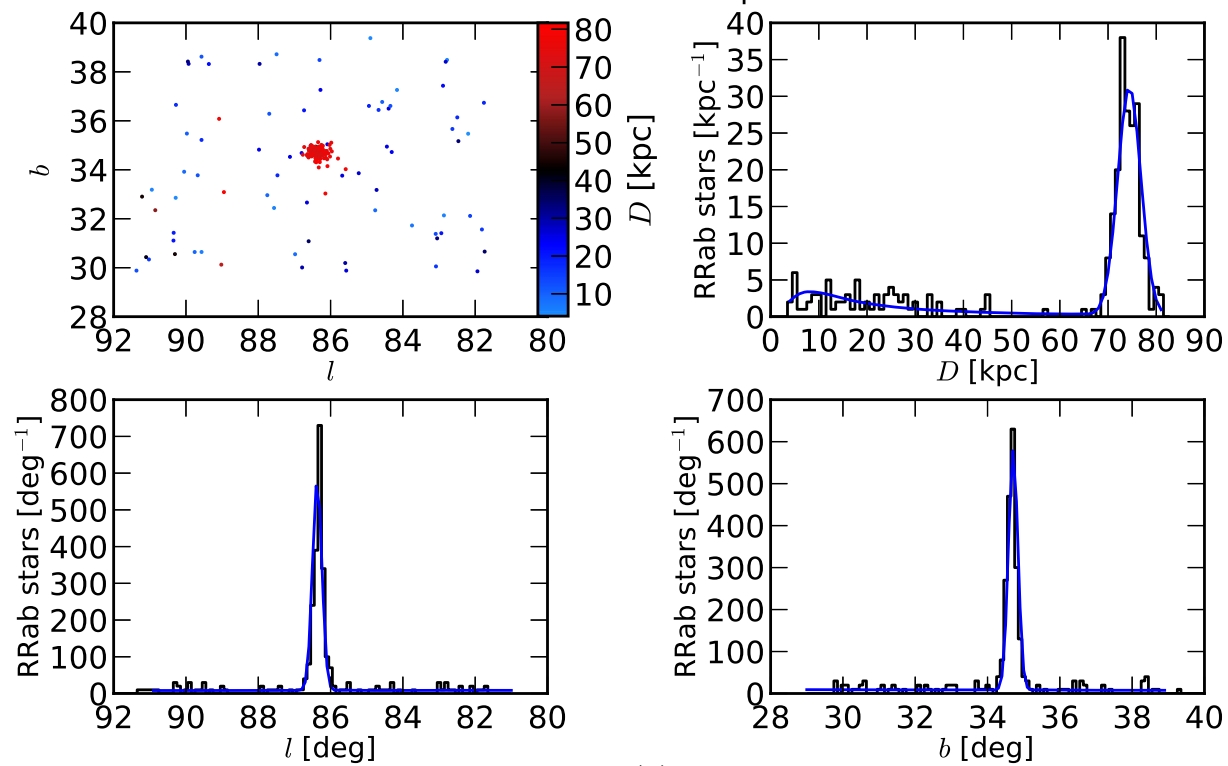

(a)
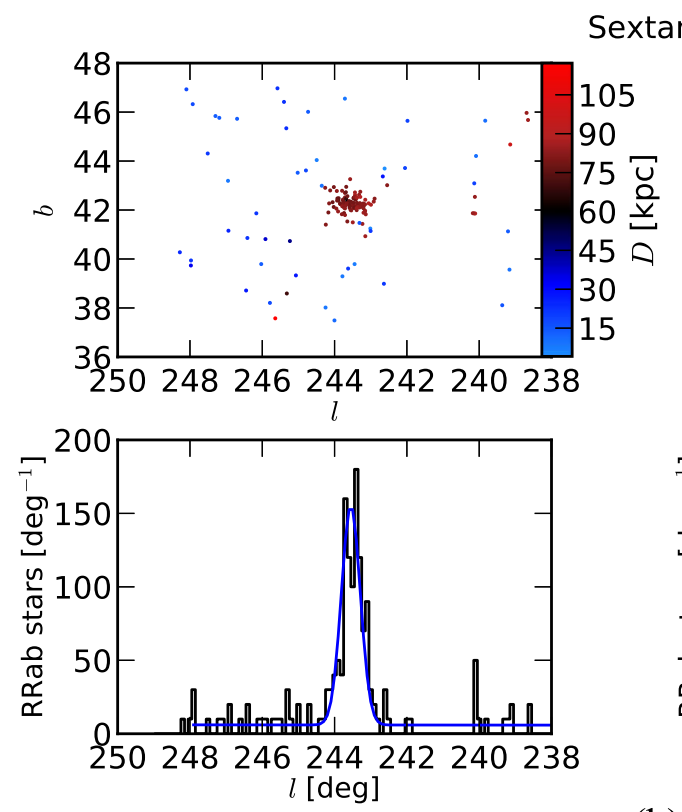

Sextans dSph
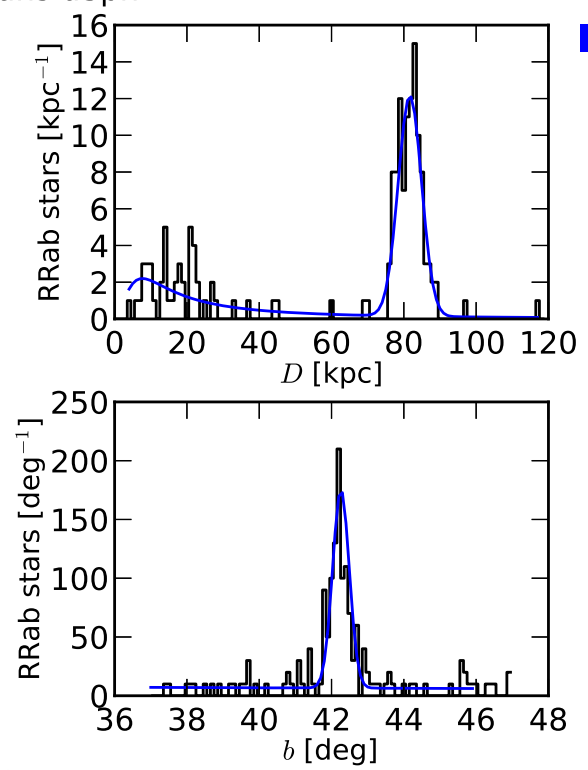

best fit parameters

with $1 \sigma$

$n=3.62_{-0.18}^{+0.17}$

$f_{*}=0.69_{-0.027}^{+0.028}$

$D=74.26_{-0.18}^{+0.18} \mathrm{kpc}$

$\sigma_{D}=2.40_{-0.15}^{+0.17} \mathrm{kpc}$

$l=86.37_{-0.01}^{+0.01} \mathrm{deg}$

$\sigma_{l}=0.13_{-0.01}^{+0.01} \mathrm{deg}$

$b=34.71_{-0.01}^{+0.01} \mathrm{deg}$

$\sigma_{b}=0.13_{-0.01}^{+0.01} \mathrm{deg}$

(b)

best fit parameters

with $1 \sigma$

$n=3.79_{-0.2}^{+0.2}$

$f_{*}=0.62_{-0.036}^{+0.04}$

$D=81.42_{-0.4}^{+0.41} \mathrm{kpc}$

$\sigma_{D}=3.24_{-0.37}^{+0.43} \mathrm{kpc}$

$l=243.55_{-0.04}^{+0.04} \mathrm{deg}$

$\sigma_{l}=0.26_{-0.03}^{+0.03} \mathrm{deg}$

$b=42.26_{-0.03}^{+0.04} \mathrm{deg}$

$\sigma_{b}=0.23_{-0.03}^{+0.04} \mathrm{deg}$

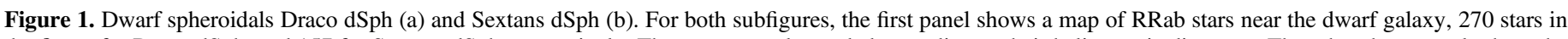

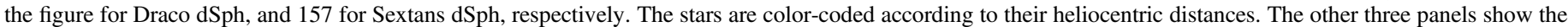
histograms in $l, b, D$ for the stars from the first panel. Overplotted is the best-fit model from Section 4 with the parameters given on the right. 


\section{Sagittarius dSph}
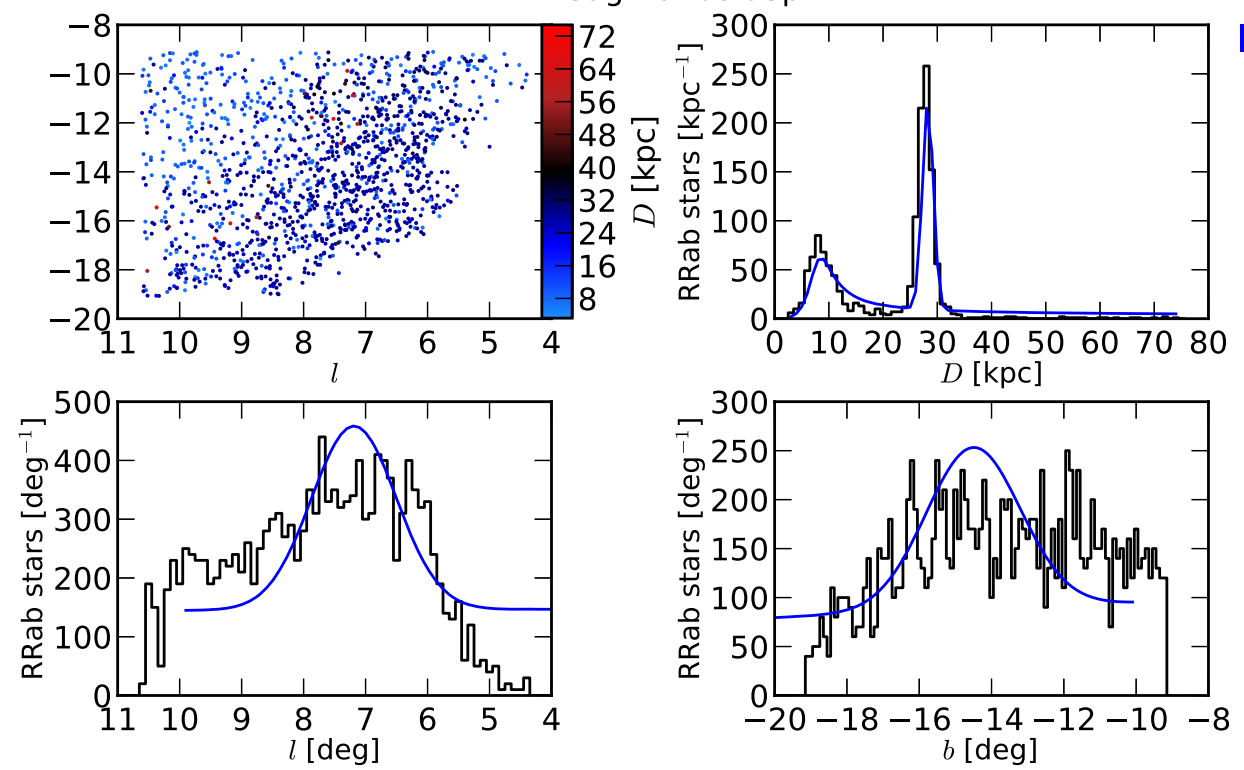

best fit parameters

with $1 \sigma$

$n=2.20_{-0.026}^{+0.027}$

$f_{*}=0.38_{-0.00038}^{+0.00085}$

$D=28.18_{-0.098}^{+0.093} \mathrm{kpc}$

$\sigma_{D}=1.01_{-0.0082}^{+0.016} \mathrm{kpc}$

$l=7.19_{-0.078}^{+0.083} \mathrm{deg}$

$\sigma_{l}=0.69_{-0.055}^{+0.052} \mathrm{deg}$

$b=-14.50_{-0.15}^{+0.18} \mathrm{deg}$

$\sigma_{b}=1.30_{-0.087}^{+0.094} \mathrm{deg}$

Figure 2. The dwarf spheroidal Sagittarius dSph. The first panel shows a map of 1413 RRab stars near the dwarf galaxy; the stars are color-coded according to their heliocentric distance. The other three panels show the histograms in $l, b, D$ for the stars from the first panel. Overplotted is the best-fit model from Section 4 with the parameters given on the right. As the Sagittarius dSph lies near the edge of the PS1 $3 \pi$ footprint, we cannot successfully fit its on-sky position $(l, b)$, but we still can fit its heliocentric distance $D$. 


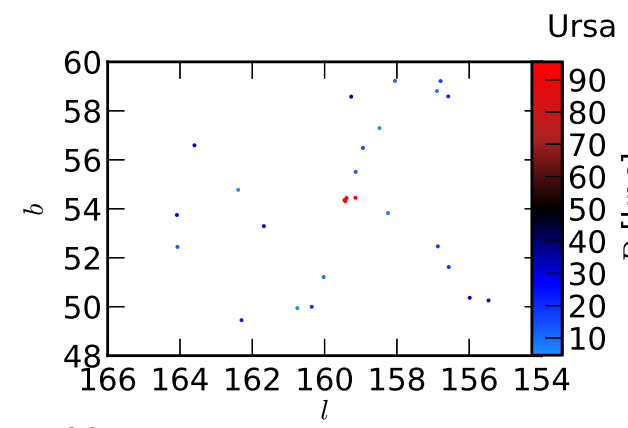

Ursa Major I dSph

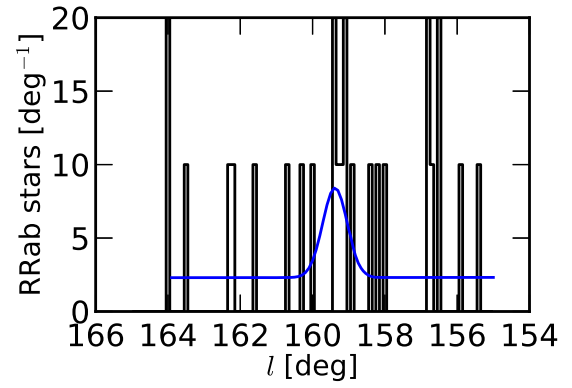

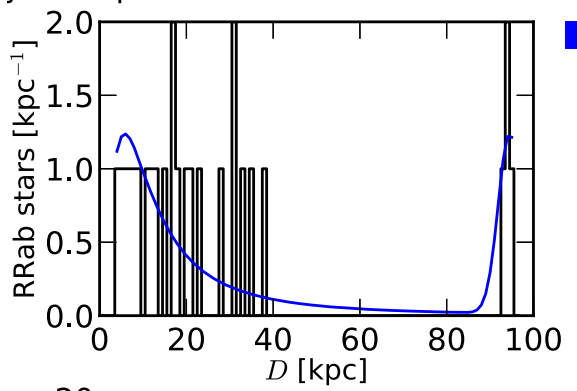

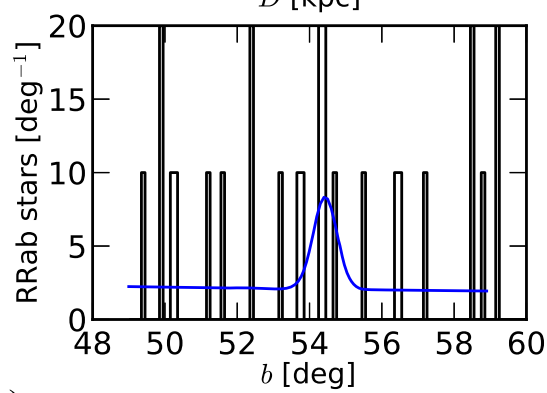

(a)
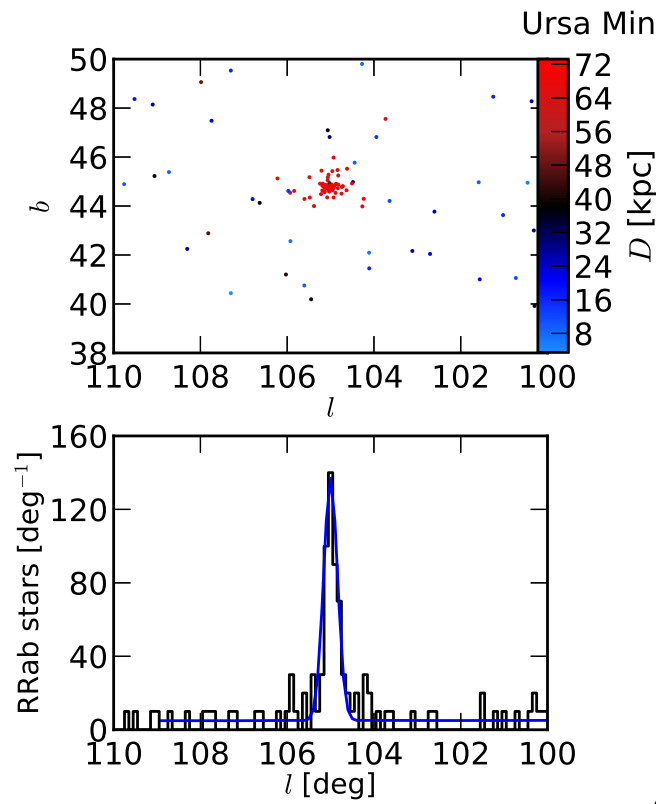
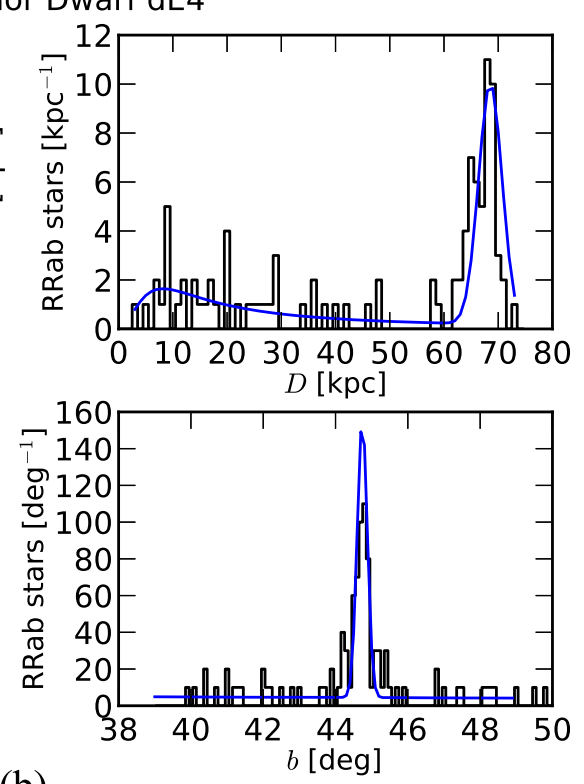

best fit parameters

with $1 \sigma$

$n=4.47_{-0.39}^{+0.31}$

$f_{*}=0.20_{-0.035}^{+0.065}$

$D=94.33_{-4.9}^{+11} \mathrm{kpc}$

$\sigma_{D}=2.59_{-1.1}^{+5.9} \mathrm{kpc}$

$l=159.38_{-1.5}^{+2.1} \mathrm{deg}$

$\sigma_{l}=0.34_{-0.2}^{+2.5} \mathrm{deg}$

$b=54.43_{-0.39}^{+2.8} \mathrm{deg}$

$\sigma_{b}=0.33_{-0.19}^{+2} \mathrm{deg}$

(b)

best fit parameters

with $1 \sigma$

$n=3.55_{--0.28}^{+0.28}$

$f_{*}=0.54_{-0.049}^{+0.054}$

$D=68.41_{-0.51}^{+0.51} \mathrm{kpc}$

$\sigma_{D}=2.17_{-0.59}^{+3.1} \mathrm{kpc}$

$l=105.00_{-0.06}^{+0.62} \mathrm{deg}$

$\sigma_{l}=0.16_{-0.03}^{+0.86} \mathrm{deg}$

$b=44.74_{-0.06}^{+0.36} \mathrm{deg}$

$\sigma_{b}=0.14_{-0.02}^{+1.4} \mathrm{deg}$

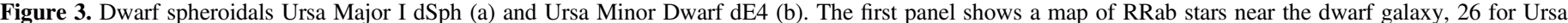

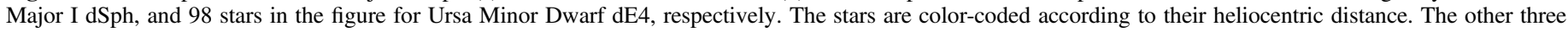

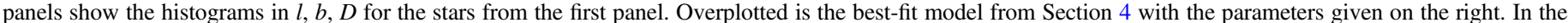

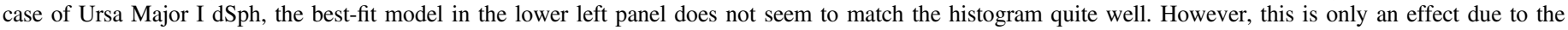
marginalization in the histogram, as it shows all sources independent of their distance, while the Gaussian is centered on the fitted ( $l, b, D$ ). 


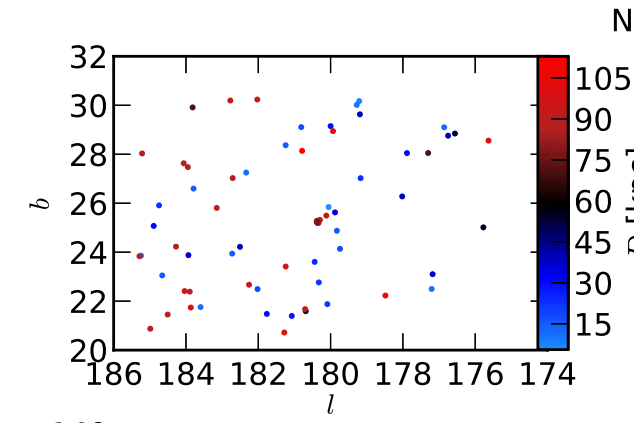

NGC 2419
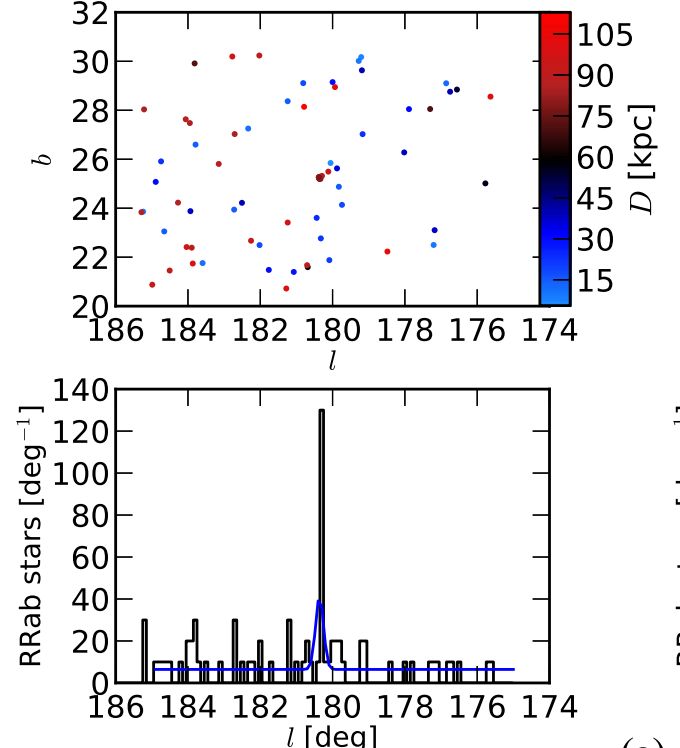

(a)
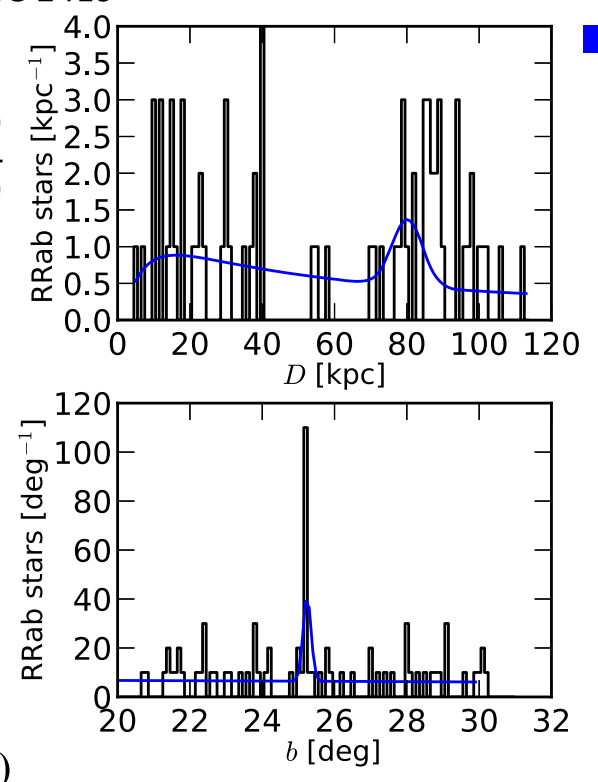

NGC 4590 (M68)
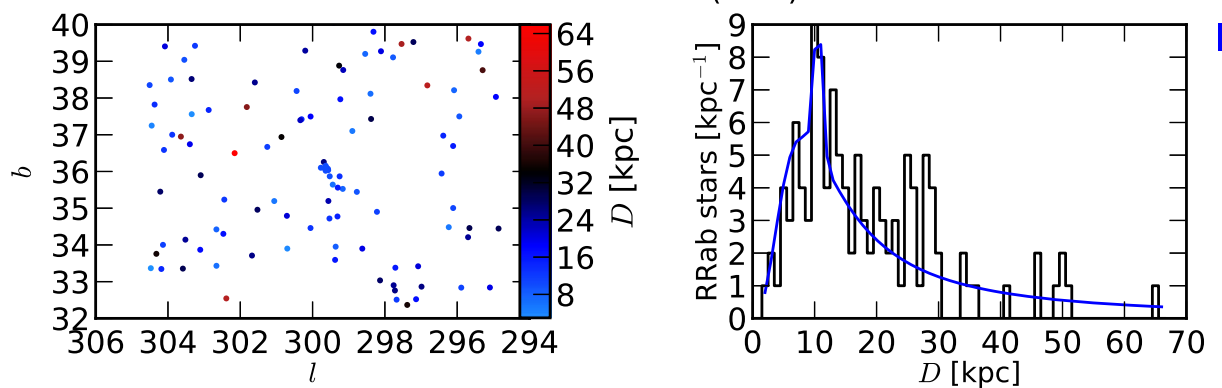

best fit parameters

with $1 \sigma$

$n=3.51_{-0.16}^{+0.13}$

$f_{*}=0.06_{-0.0099}^{+0.017}$

$D=10.48_{-0.28}^{+0.26} \mathrm{kpc}$

$\sigma_{D}=0.66_{-0.13}^{+1.3} \mathrm{kpc}$

$l=299.62_{-0.08}^{+0.12} \mathrm{deg}$

$\sigma_{l}=0.15_{-0.04}^{+0.68} \mathrm{deg}$

$b=36.07_{-0.11}^{+0.1} \mathrm{deg}$

$\sigma_{b}=0.15_{-0.04}^{+0.65} \mathrm{deg}$

(b)

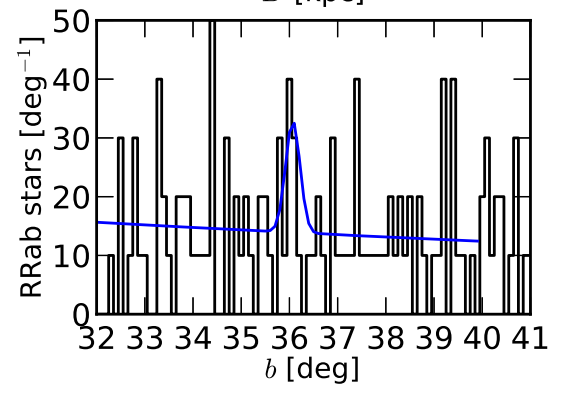

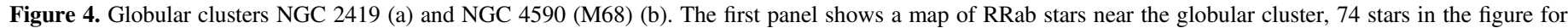

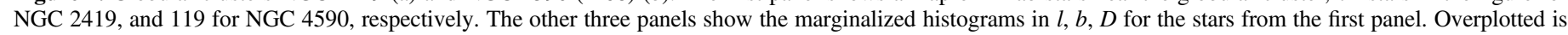

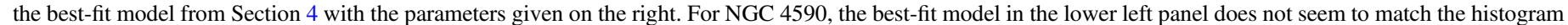

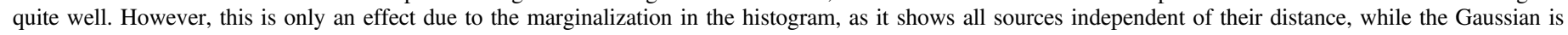
centered on the fitted $(l, b, D)$. 


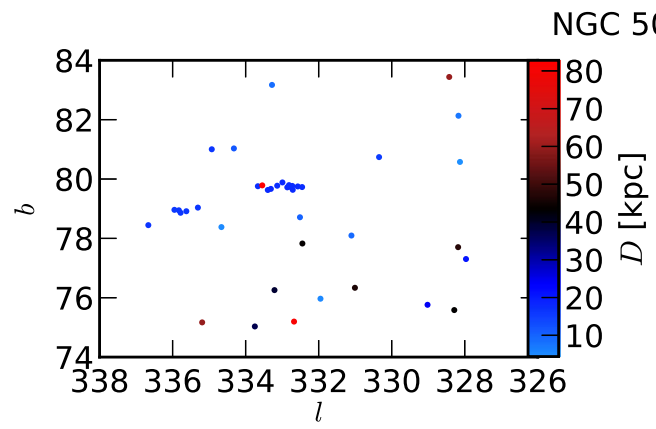

NGC 5024 (M53)
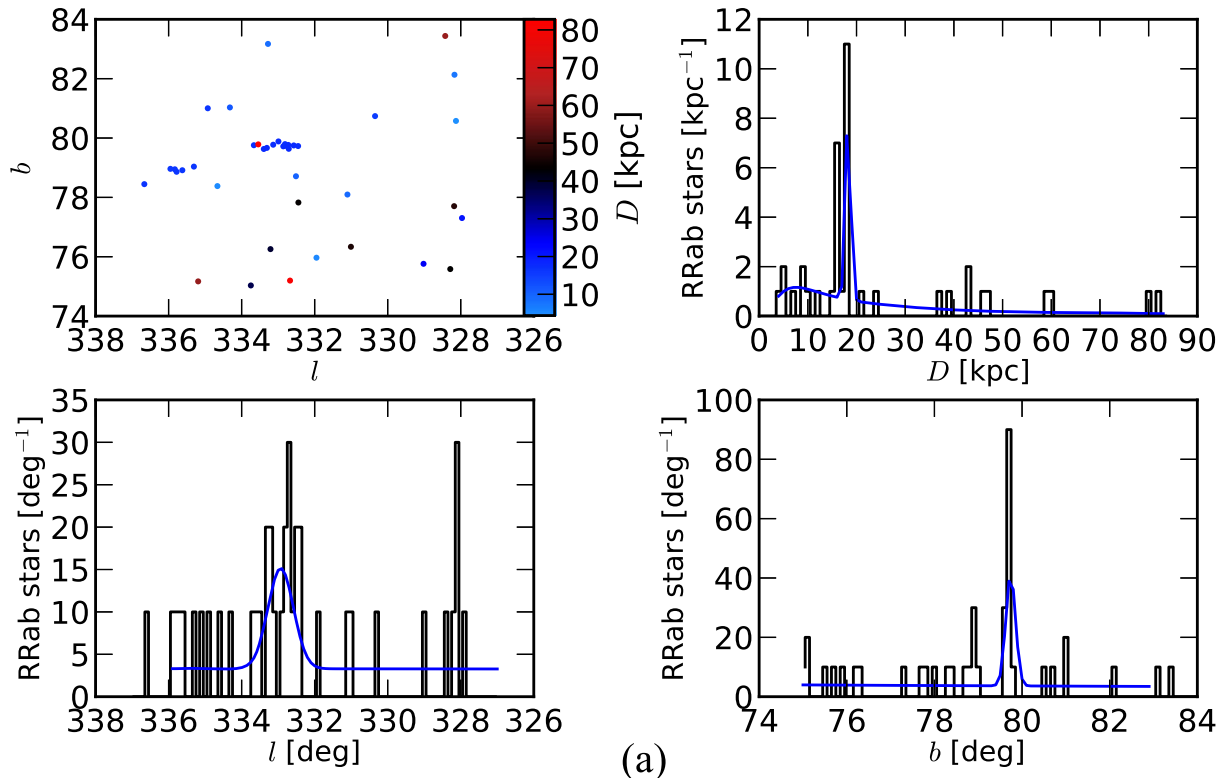

best fit parameters

with $1 \sigma$

$n=3.32_{-0.3}^{+0.29}$

$f_{*}=0.26_{-0.078}^{+0.078}$

$D=18.25_{-0.14}^{+0.13} \mathrm{kpc}$

$\sigma_{D}=0.55_{-0.03}^{+0.08} \mathrm{kpc}$

$l=332.93_{-0.12}^{+0.12} \mathrm{deg}$

$\sigma_{l}=0.35_{-0.08}^{+0.12} \mathrm{deg}$

$b=79.74_{-0.04}^{+0.04} \mathrm{deg}$

$\sigma_{b}=0.11_{-0.01}^{+0.02} \mathrm{deg}$

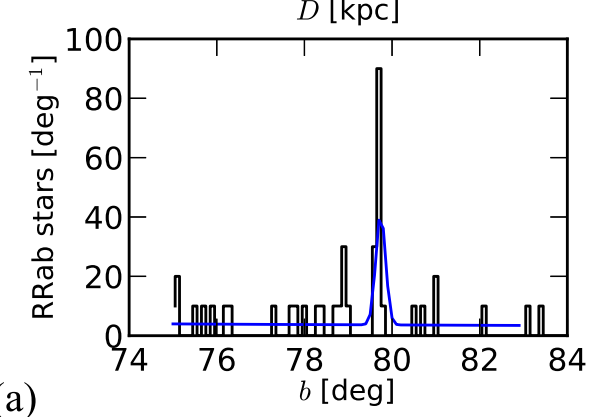

(a)

NGC 5053
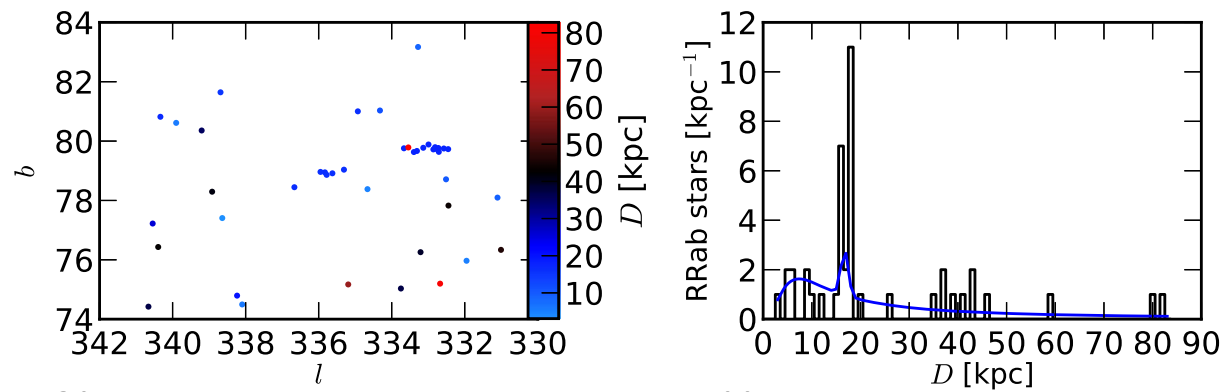

best fit parameters

with $1 \sigma$

$n=3.39_{-0.25}^{+0.2}$

$f_{*}=0.08_{-0.022}^{+0.054}$

$D=16.66_{-0.26}^{+0.28} \mathrm{kpc}$

$\sigma_{D}=0.75_{-0.2}^{+3.7} \mathrm{kpc}$

$l=335.78_{-0.18}^{+0.19} \mathrm{deg}$

$\sigma_{l}=0.30_{-0.16}^{+1.1} \mathrm{deg}$

$b=78.93_{-0.14}^{+0.16} \mathrm{deg}$

$\sigma_{b}=0.18_{-0.06}^{+1.1} \mathrm{deg}$
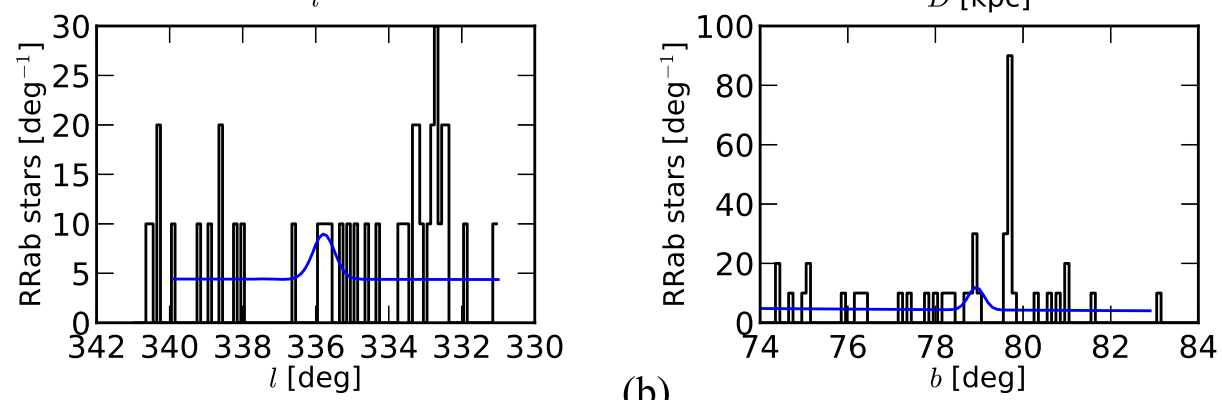

(b)

Figure 5. Globular clusters NGC 5024 (M53) (a) and NGC 5053 (b). The first panel shows a map of RRab stars near the globular cluster, 40 stars in the figure for NGC 5024, and 75 stars for NGC 5272, respectively. The stars are color-coded according to their heliocentric distance. In the Cartesian projection, both NGC 5024 and NGC 5053 appear to be elongated in $l$ direction because of its high latitude. The other three panels show the histograms in $l, b, D$ for the stars from the first panel. Overplotted is the best-fit model from Section 4 with the parameters given on the right. 
NGC 5272 (M3)
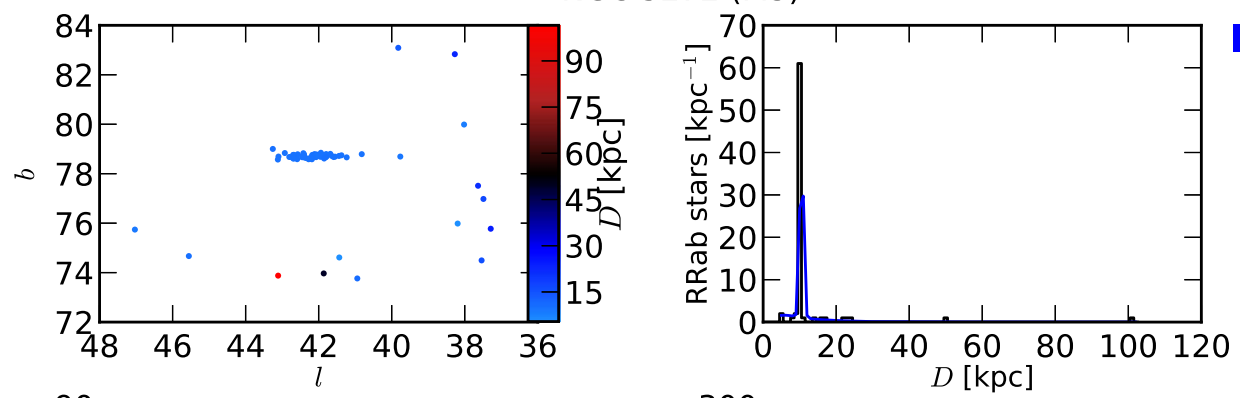

best fit parameters

with $1 \sigma$

$n=4.36_{-0.38}^{+0.43}$

$f_{*}=0.74_{-0.09}^{+0.059}$

$D=10.48_{-0.07}^{+0.07} \mathrm{kpc}$

$\sigma_{D}=0.51_{-0.01}^{+0.02} \mathrm{kpc}$

$l=42.20_{-0.07}^{+0.08} \mathrm{deg}$

$\sigma_{l}=0.45_{-0.05}^{+0.05} \mathrm{deg}$

$b=78.71_{-0.02}^{+0.02} \mathrm{deg}$

$\sigma_{b}=0.10_{-0.002}^{+0.01} \mathrm{deg}$

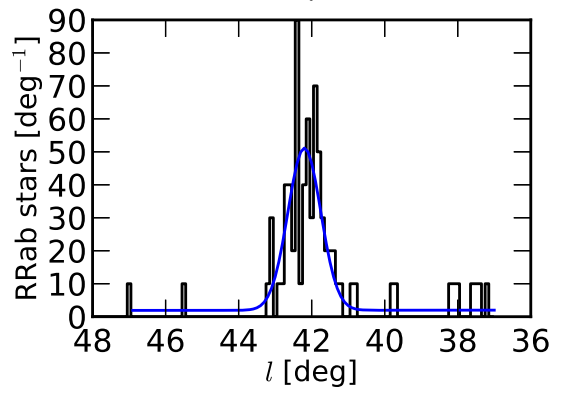

(a)

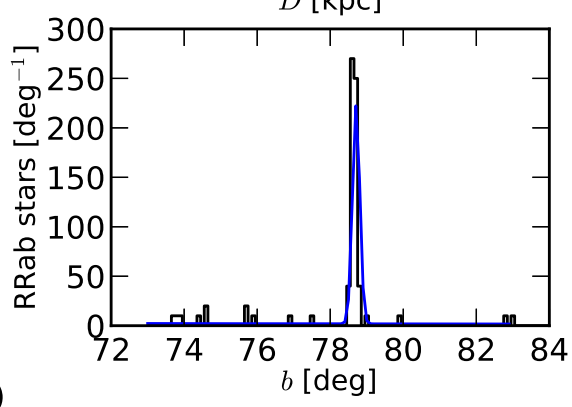

NGC 5466
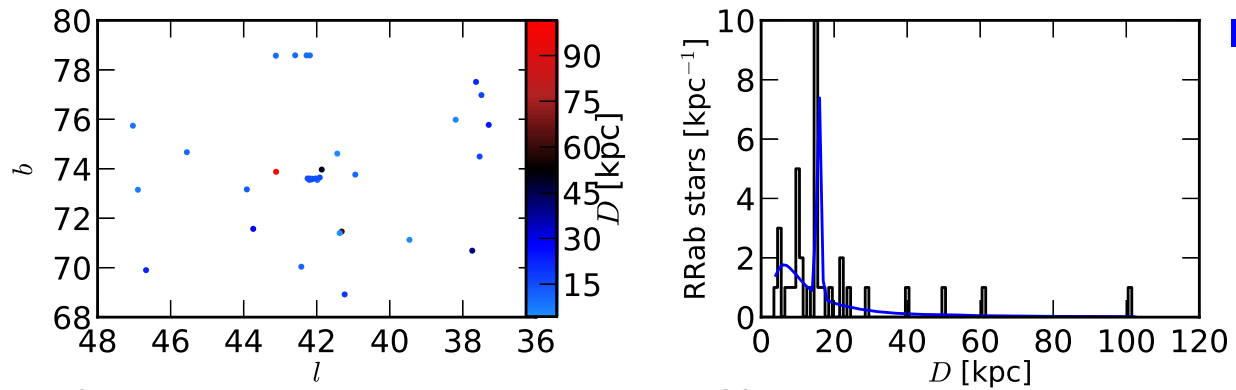

best fit parameters

with $1 \sigma$

$n=4.07_{-0.29}^{+0.33}$

$f_{*}=0.27_{-0.064}^{+0.073}$

$D=15.76_{-0.14}^{+0.14} \mathrm{kpc}$

$\sigma_{D}=0.54_{-0.03}^{+0.07} \mathrm{kpc}$

$l=42.13_{-0.04}^{+0.04} \mathrm{deg}$

$\sigma_{l}=0.12_{-0.02}^{+0.03} \mathrm{deg}$

$b=73.59_{-0.03}^{+0.04} \mathrm{deg}$

$\sigma_{b}=0.11_{-0.01}^{+0.02} \mathrm{deg}$
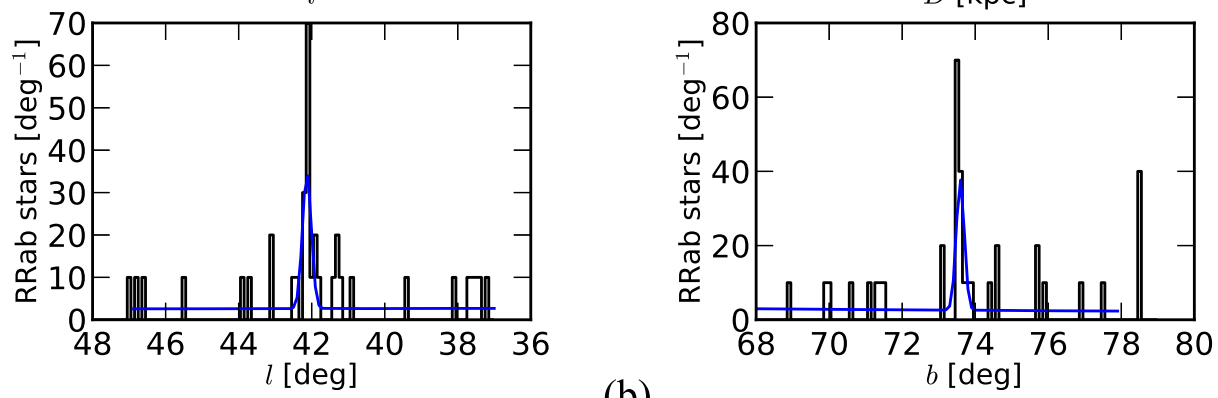

(b)

Figure 6. Globular clusters NGC 5272 (M3) (a) and NGC 5466 (M5) (b). The first panel shows a map of RRab stars near the globular cluster, 75 in the figure for NGC 5272, and 36 for NGC 5904, respectively. In the Cartesian projection, both NGC 5272 and NGC 5466 appear to be elongated in the $l$ direction because of its high latitude. The other three panels show the histograms in $l, b, D$ for the stars from the first panel. Overplotted is the best-fit model from Section 4 with the parameters given on the right. 


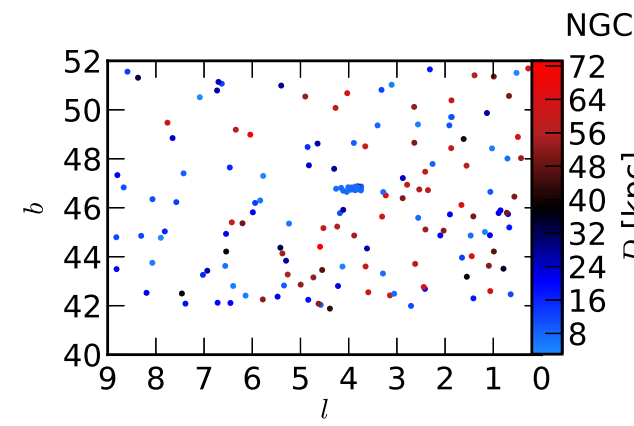

NGC 5904 (M5)
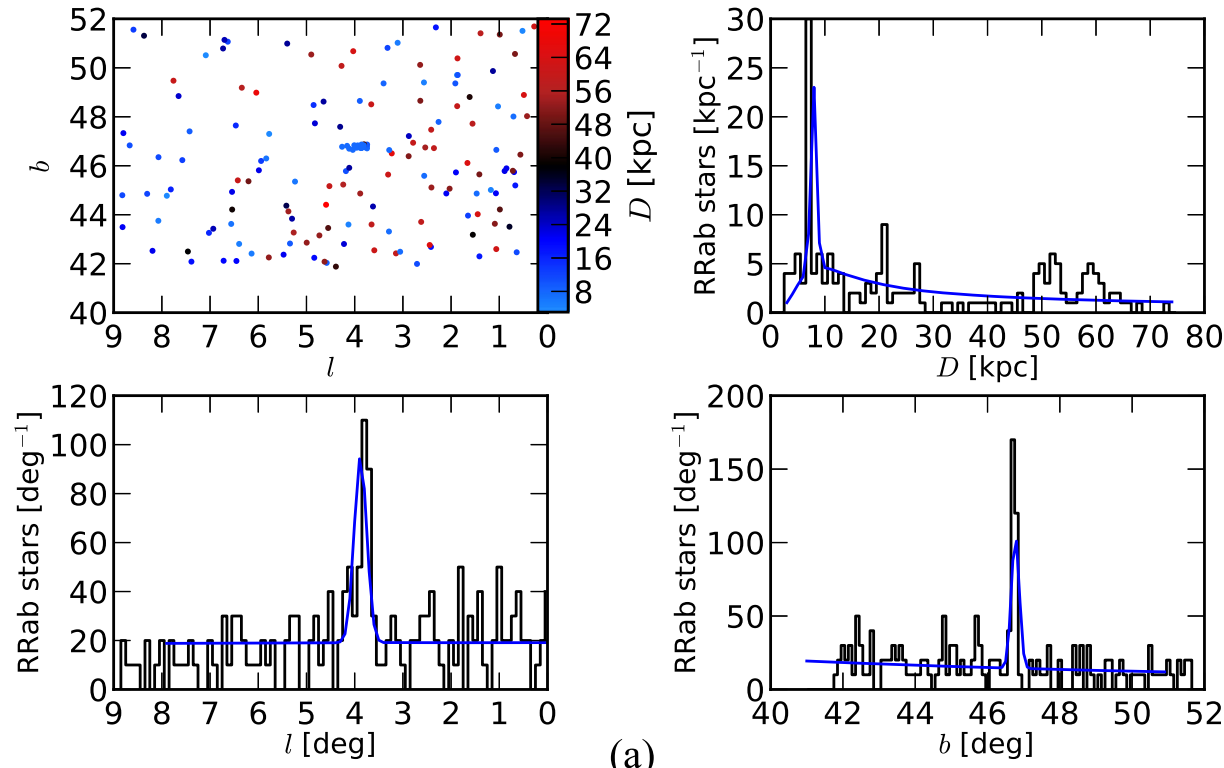

best fit parameters

with $1 \sigma$

$n=2.54_{-0.13}^{+0.13}$

$f_{*}=0.14_{-0.038}^{+0.028}$

$D=7.87_{-0.19}^{+0.19} \mathrm{kpc}$

$\sigma_{D}=0.53_{-0.02}^{+0.08} \mathrm{kpc}$

$l=3.88_{-0.04}^{+0.03} \mathrm{deg}$

$\sigma_{l}=0.13_{-0.02}^{+0.04} \mathrm{deg}$

$b=46.77_{-0.02}^{+0.03} \mathrm{deg}$

$\sigma_{b}=0.11_{-0.005}^{+0.02} \mathrm{deg}$

(a)

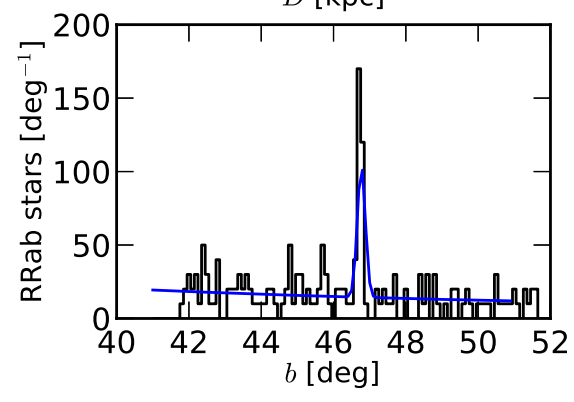

NGC 6229
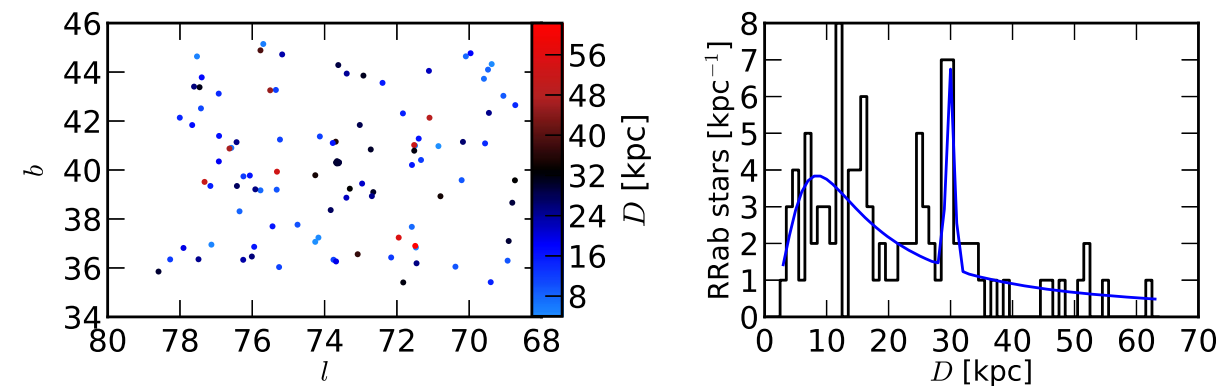

best fit parameters

with $1 \sigma$

$n=3.34_{-0.18}^{+0.17}$

$f_{*}=0.08_{-0.021}^{+0.027}$

$D=29.94_{-0.19}^{+0.17} \mathrm{kpc}$

$\sigma_{D}=0.61_{-0.08}^{+0.19} \mathrm{kpc}$

$l=73.64_{-0.04}^{+0.04} \mathrm{deg}$

$\sigma_{l}=0.11_{-0.01}^{+0.02} \mathrm{deg}$

$b=40.31_{-0.04}^{+0.04} \mathrm{deg}$

$\sigma_{b}=0.11_{-0.01}^{+0.03} \mathrm{deg}$

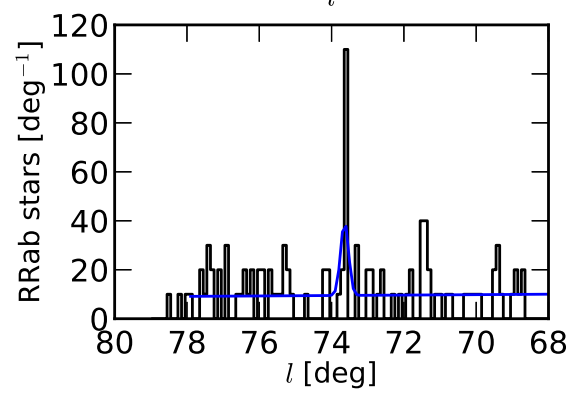

(b)

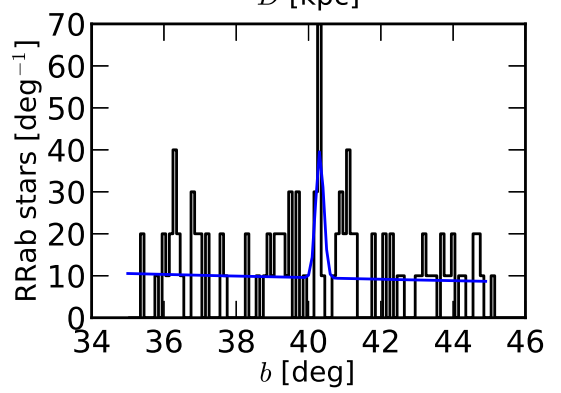

Figure 7. Globular clusters NGC 5904 (M5) (a) and NGC 6229 (b). The first panel shows a map of RRab stars near the globular cluster, 177 in the figure for NGC 5904, and 104 for NGC 6229, respectively. The other three panels show the histograms in $l, b, D$ for the stars from the first panel. Overplotted is the best-fit model from Section 4 with the parameters given on the right. For NGC 5904, the best-fit model in the lower left panel does not seem to match the histogram quite well. However, this is only an effect due to the marginalization in the histogram, as it shows all sources independent of their distance, while the Gaussian is centered on the fitted $(l, b, D)$. 

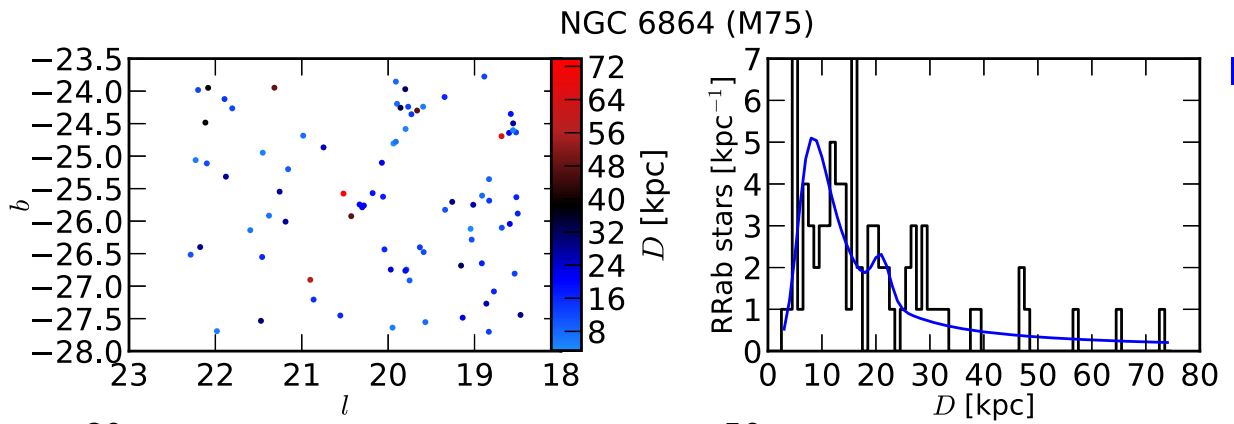

best fit parameters

with $1 \sigma$

$n=2.96_{-0.054}^{+0.055}$

$f_{*}=0.05_{-0.0011}^{+0.0027}$

$D=20.79_{-0.35}^{+0.32} \mathrm{kpc}$

$\sigma_{D}=1.59_{-0.93}^{+4.3} \mathrm{kpc}$

$l=20.28_{-0.23}^{+0.25} \mathrm{deg}$

$\sigma_{l}=0.25_{-0.13}^{+1.5} \mathrm{deg}$

$b=-25.76_{-0.28}^{+0.2} \mathrm{deg}$

$\sigma_{b}=0.34_{-0.21}^{+2.7} \mathrm{deg}$
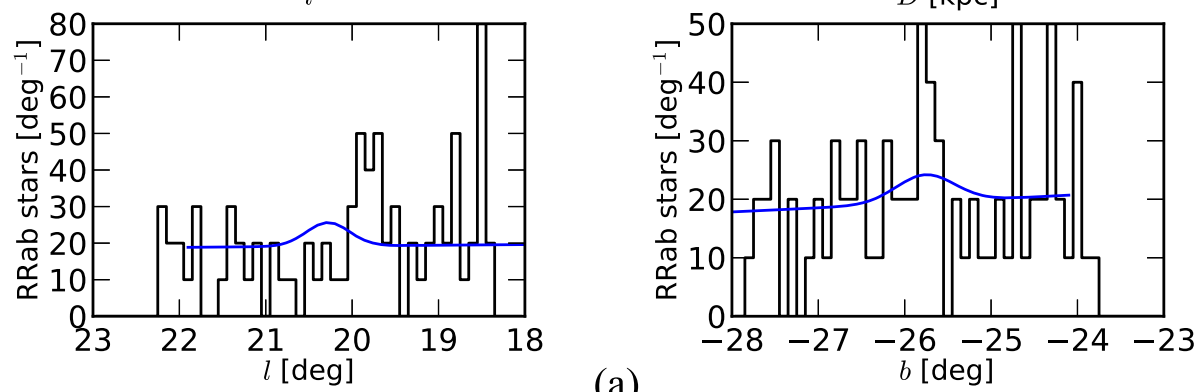

(a)

\section{NGC 6934}
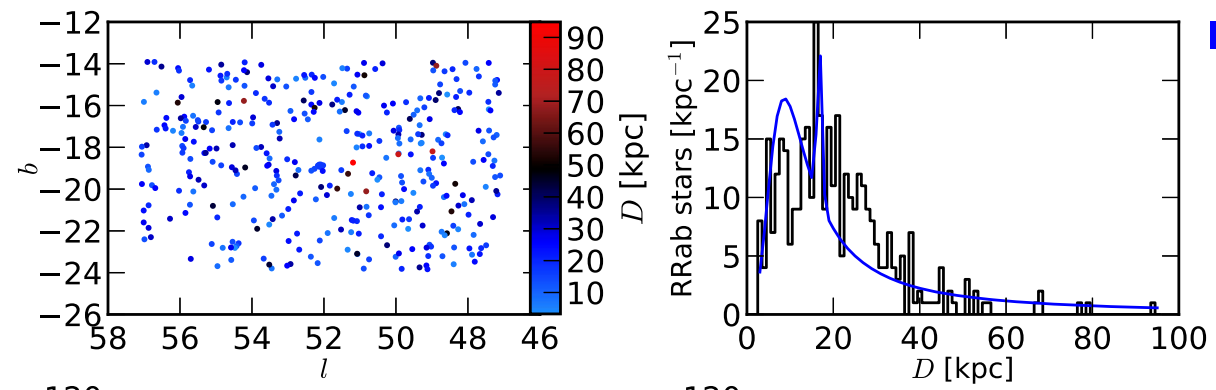

best fit parameters

with $1 \sigma$

$n=3.38_{-0.065}^{+0.067}$

$f_{*}=0.05_{-0.0028}^{+0.0046}$

$D=16.77_{-0.21}^{+0.21} \mathrm{kpc}$

$\sigma_{D}=0.57_{-0.05}^{+0.16} \mathrm{kpc}$

$l=52.12_{-0.04}^{+0.04} \mathrm{deg}$

$\sigma_{l}=0.11_{-0.01}^{+0.03} \mathrm{deg}$

$b=-18.87_{-0.04}^{+0.03} \mathrm{deg}$

$\sigma_{b}=0.11_{-0.01}^{+0.02} \mathrm{deg}$
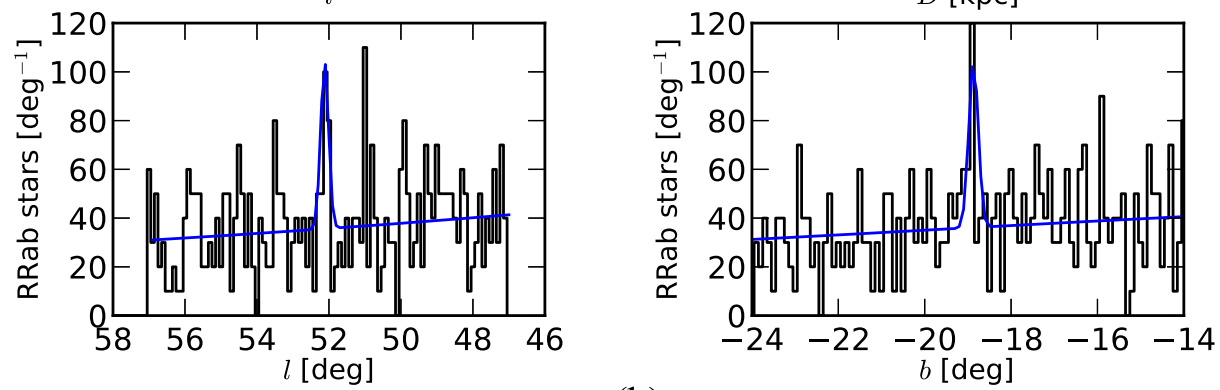

(b)

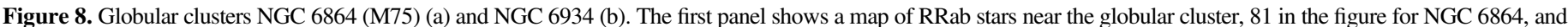

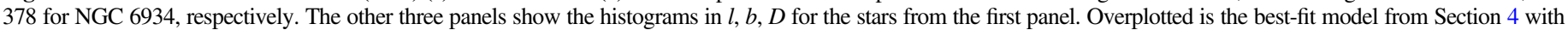

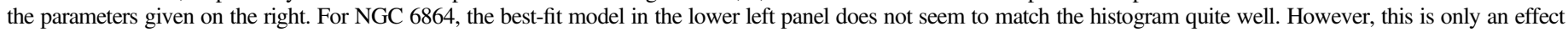
due to the marginalization in the histogram, as it shows all sources independent of their distance, while the Gaussian is centered on the fitted ( $l, b, D$ ). 

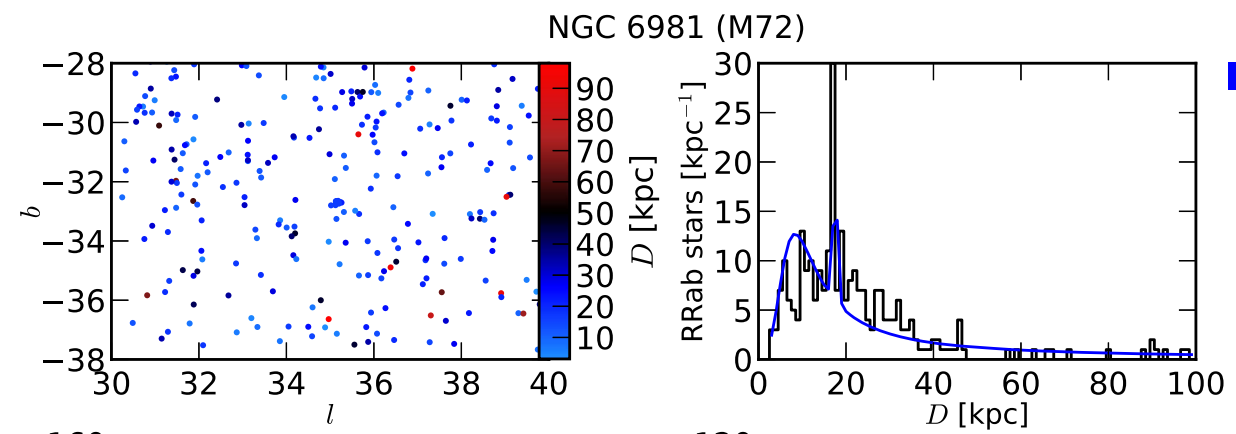

best fit parameters

with $1 \sigma$

$n=3.14_{-0.084}^{+0.077}$

$f_{*}=0.06_{-0.007}^{+0.012}$

$D=17.51_{-0.17}^{+0.15} \mathrm{kpc}$

$\sigma_{D}=0.56_{-0.05}^{+0.11} \mathrm{kpc}$

$l=35.16_{-0.03}^{+0.03} \mathrm{deg}$

$\sigma_{l}=0.11_{-0.01}^{+0.01} \mathrm{deg}$

$b=-32.70_{-0.03}^{+0.03} \mathrm{deg}$
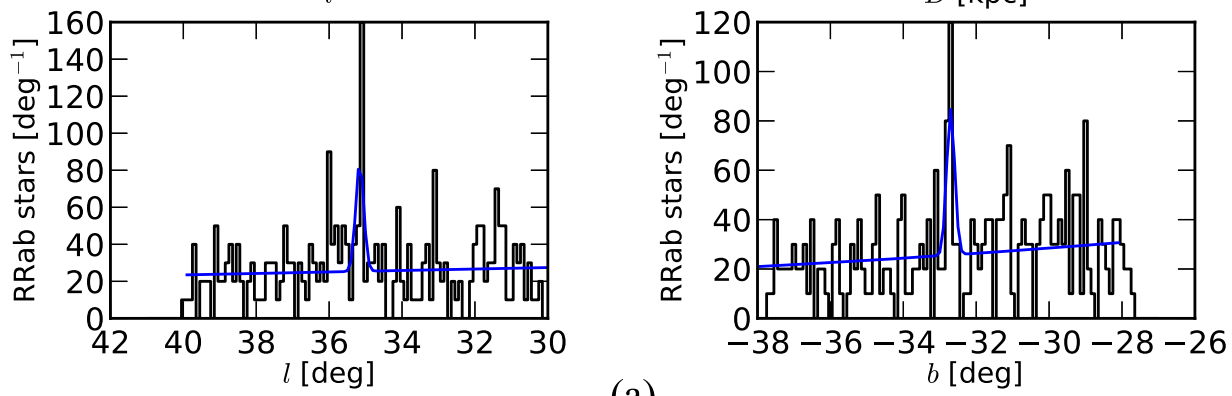

(a)

$\sigma_{b}=0.11_{-0.01}^{+0.01} \mathrm{deg}$
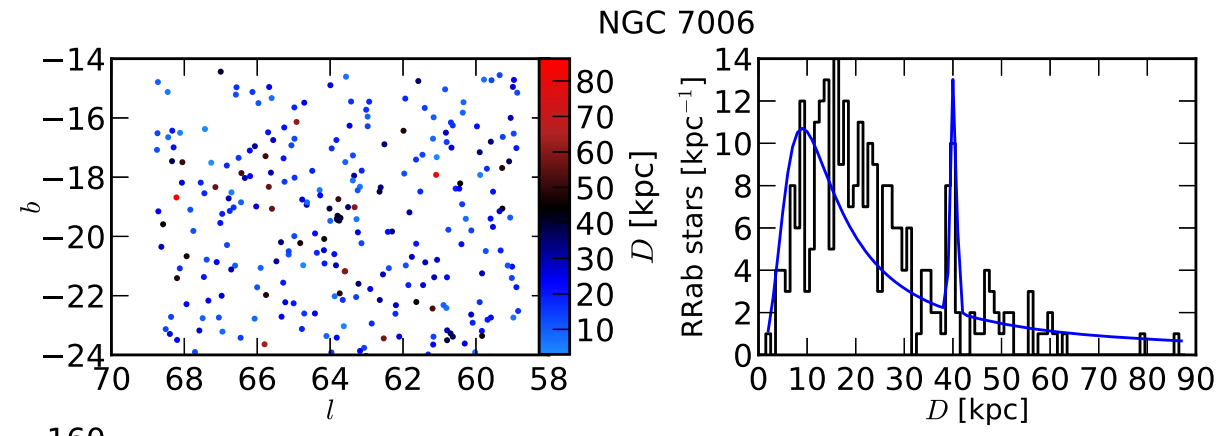

best fit parameters

with $1 \sigma$

$n=3.33_{-0.09}^{+0.092}$

$f_{*}=0.06_{-0.0084}^{+0.012}$

$D=40.12_{-0.15}^{+0.16} \mathrm{kpc}$

$\sigma_{D}=0.59_{-0.06}^{+0.11} \mathrm{kpc}$

$l=63.78_{-0.03}^{+0.03} \mathrm{deg}$

$\sigma_{l}=0.11_{-0.004}^{+0.01} \mathrm{deg}$

$b=-19.39_{-0.03}^{+0.03} \mathrm{deg}$
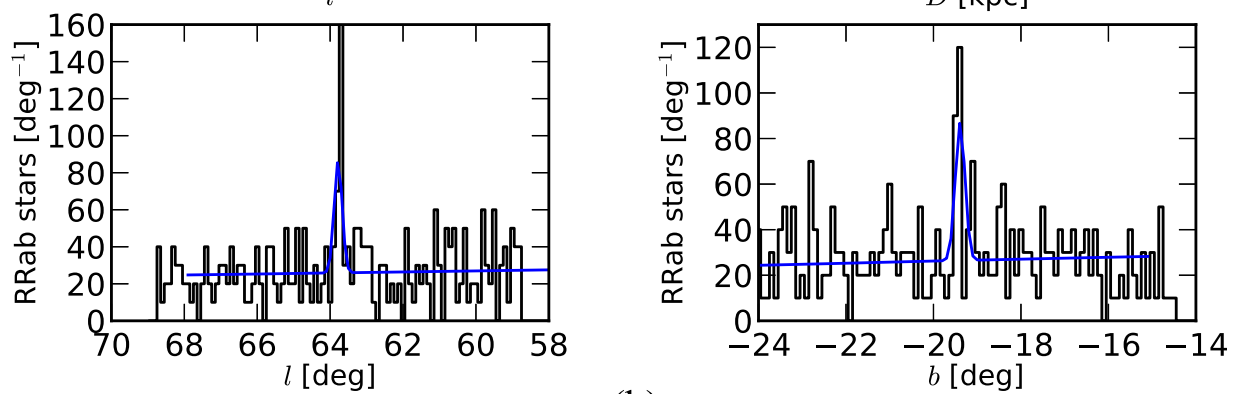

(b)

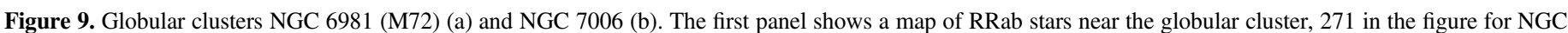

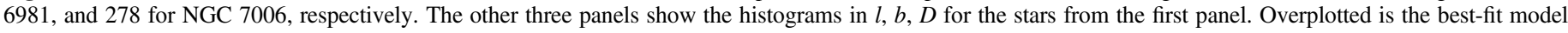
from Section 4 with the parameters given on the right. 

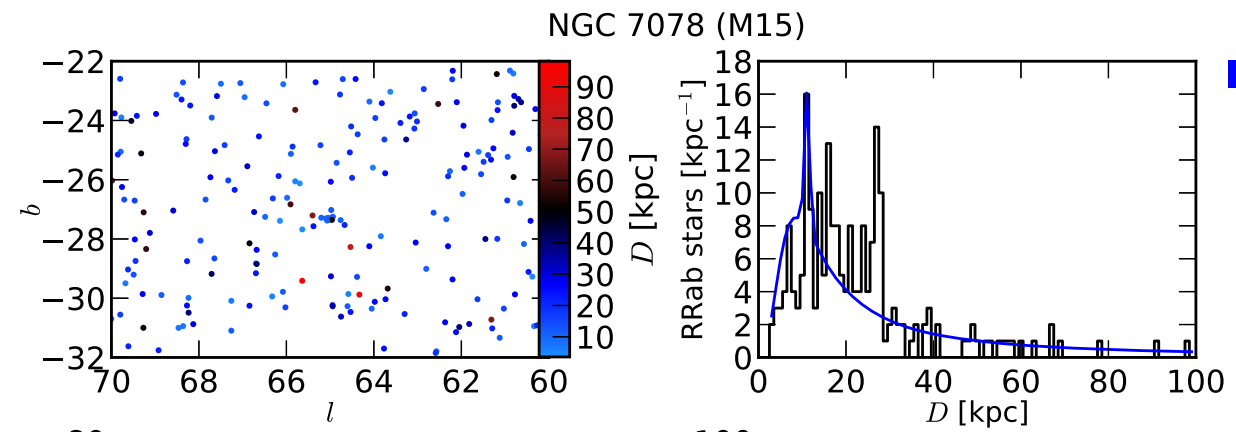

best fit parameters

with $1 \sigma$

$n=3.46_{-0.086}^{+0.099}$

$f_{*}=0.06_{-0.0081}^{+0.014}$

$D=11.07_{-0.22}^{+0.24} \mathrm{kpc}$

$\sigma_{D}=0.60_{-0.08}^{+2.7} \mathrm{kpc}$

$l=65.03_{-0.08}^{+0.11} \mathrm{deg}$

$\sigma_{l}=0.15_{-0.04}^{+1.1} \mathrm{deg}$

$b=-27.32_{-0.08}^{+0.07} \mathrm{deg}$

$\sigma_{b}=0.13_{-0.03}^{+0.95} \mathrm{deg}$

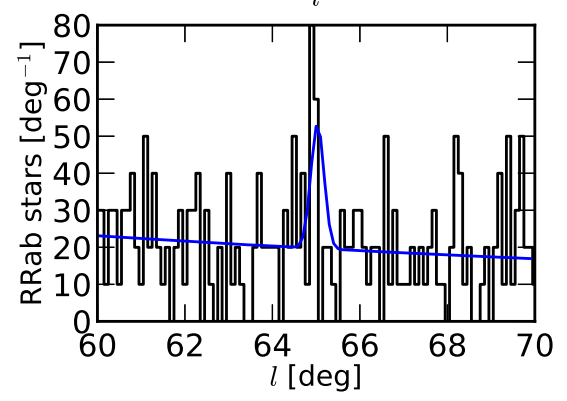

(a)

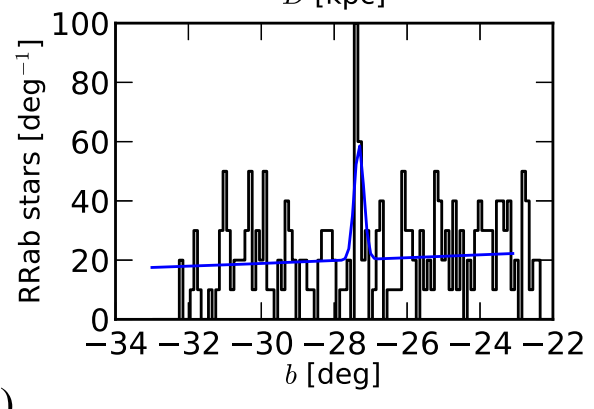

NGC 7089 (M2)
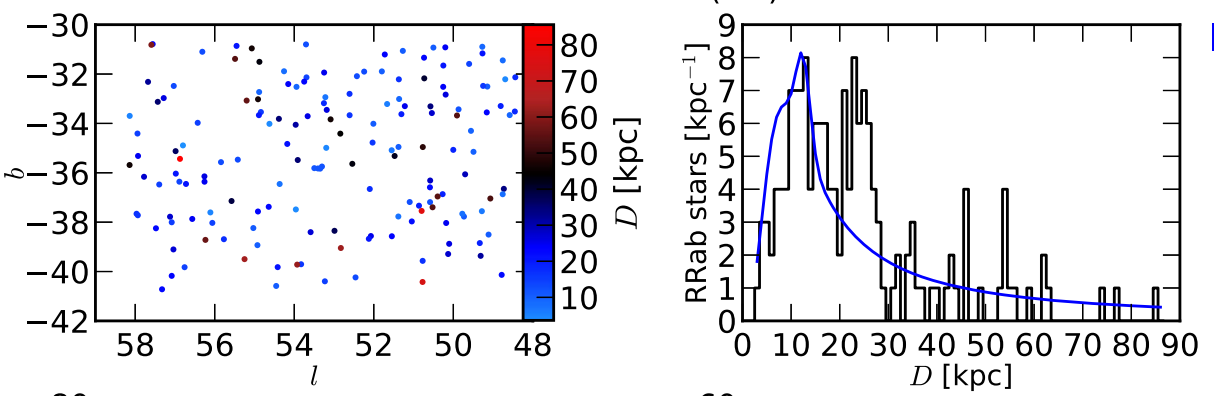

best fit parameters

with $1 \sigma$

$n=3.24_{-0.11}^{+0.11}$

$f_{*}=0.06_{-0.0044}^{+0.0077}$

$D=12.11_{-0.28}^{+0.39} \mathrm{kpc}$

$\sigma_{D}=1.47_{-0.84}^{+6.3} \mathrm{kpc}$

$l=53.46_{-0.25}^{+0.22} \mathrm{deg}$

$\sigma_{l}=0.35_{-0.21}^{+2.4} \mathrm{deg}$

$b=-35.81_{-0.29}^{+0.29} \mathrm{deg}$

$\sigma_{b}=0.62_{-0.47}^{+3.6} \mathrm{deg}$
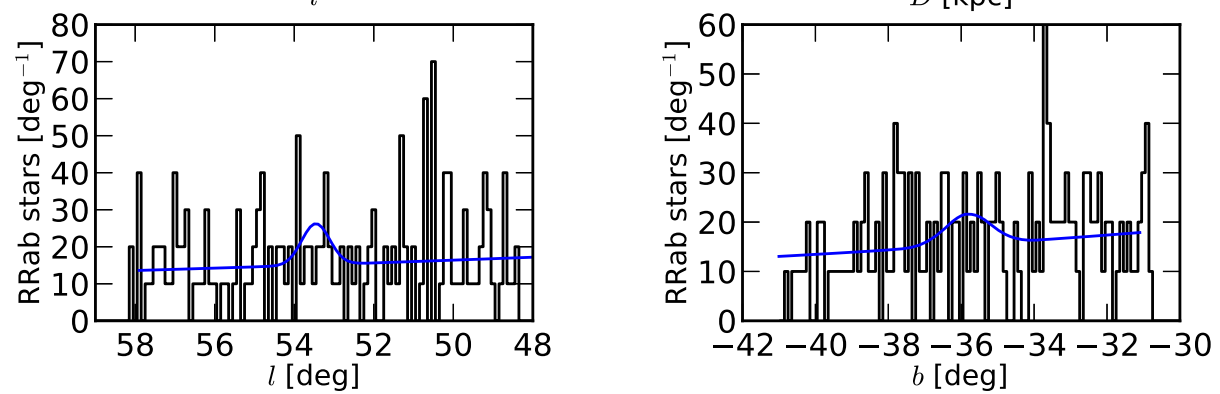

(b)

Figure 10. The globular clusters NGC 7078 (M15) (a) and NGC 7089 (M2) (b). The first panel shows a map of RRab stars near the globular cluster, 211 in the figure for NGC 7078, and 163 for NGC 7089, respectively. The other three panels show the histograms in $l, b, D$ for the stars from the first panel. Overplotted is the best-fit model from Section 4 with the parameters given on the right. For NGC 7089, the best-fit model in the lower left panel does not seem to match the histogram quite well. However, this is only an effect due to the marginalization in the histogram, as it shows all sources independent of their distance, while the Gaussian is centered on the fitted $(l, b, D)$. 
Pal 3
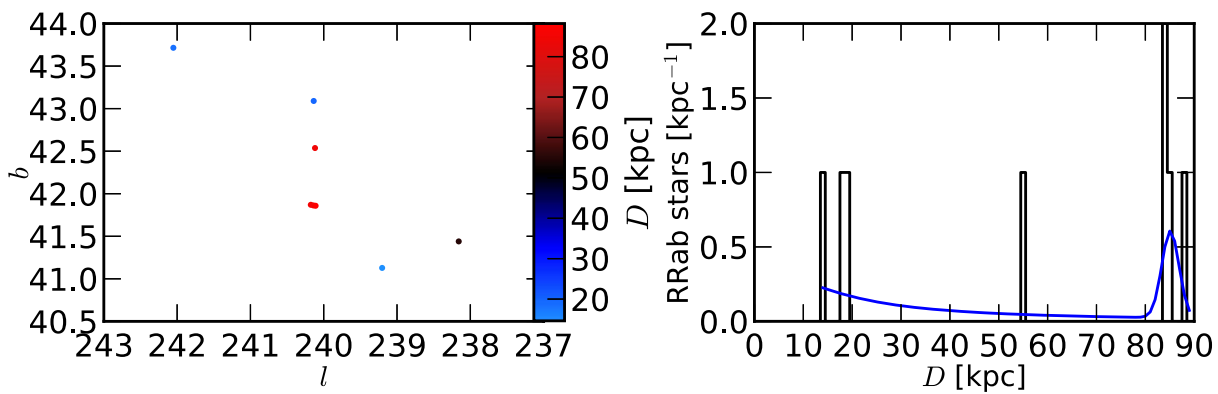

best fit parameters

with $1 \sigma$

$n=3.65_{-1.2}^{+0.92}$

$f_{*}=0.32_{-0.18}^{+0.18}$

$D=85.05_{-0.34}^{+0.32} \mathrm{kpc}$

$\sigma_{D}=1.76_{-1.1}^{+1.8} \mathrm{kpc}$

$l=240.14_{-0.09}^{+0.1} \mathrm{deg}$

$\sigma_{l}=0.14_{-0.03}^{+0.17} \mathrm{deg}$

$b=41.95_{-0.18}^{+0.21} \mathrm{deg}$

$\sigma_{b}=0.34_{-0.17}^{+0.31} \mathrm{deg}$

(a)

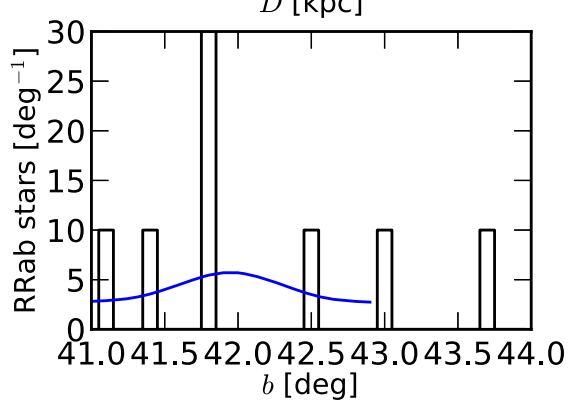

Pal 5
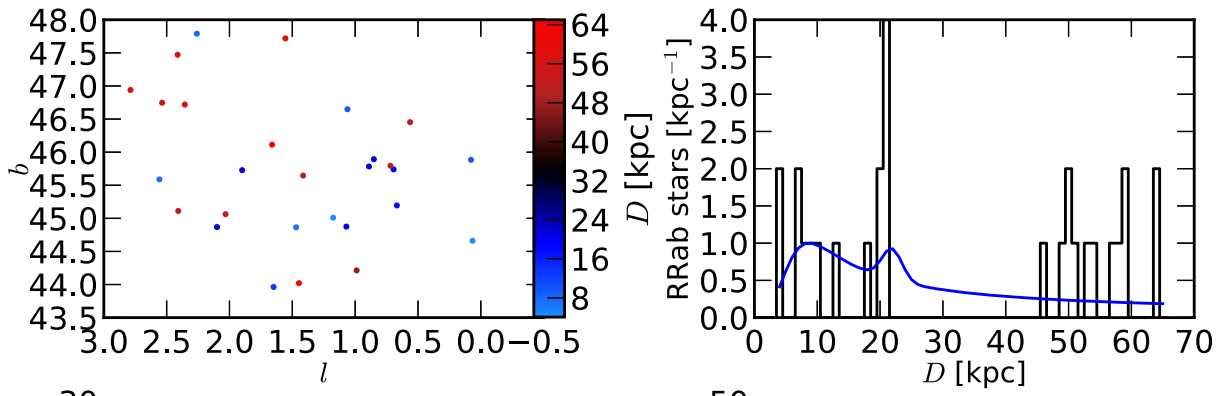

best fit parameters

with $1 \sigma$

$n=2.68_{-0.11}^{+0.098}$

$f_{*}=0.06_{-0.0038}^{+0.0074}$

$D=21.66_{-0.3}^{+0.33} \mathrm{kpc}$

$\sigma_{D}=1.61_{-0.98}^{+7.5} \mathrm{kpc}$

$l=0.87_{-0.27}^{+0.29} \mathrm{deg}$

$\sigma_{l}=0.58_{-0.43}^{+2.8} \mathrm{deg}$

$b=45.80_{-0.28}^{+0.26} \mathrm{deg}$

$\sigma_{b}=0.31_{-0.19}^{+2.4} \mathrm{deg}$

(b)

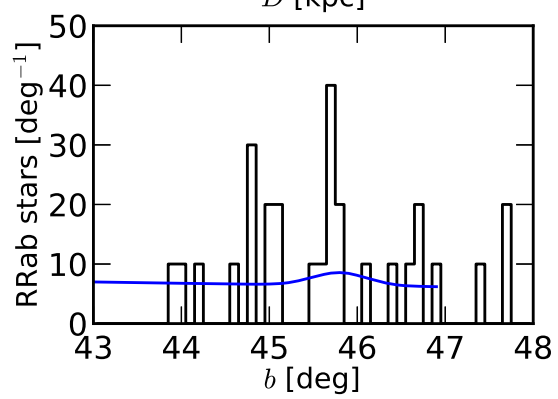

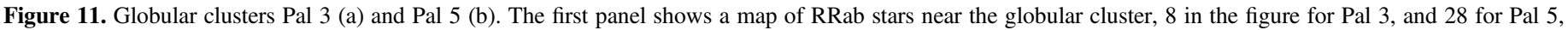

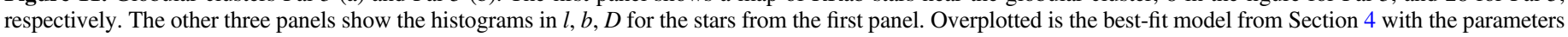
given on the right. 

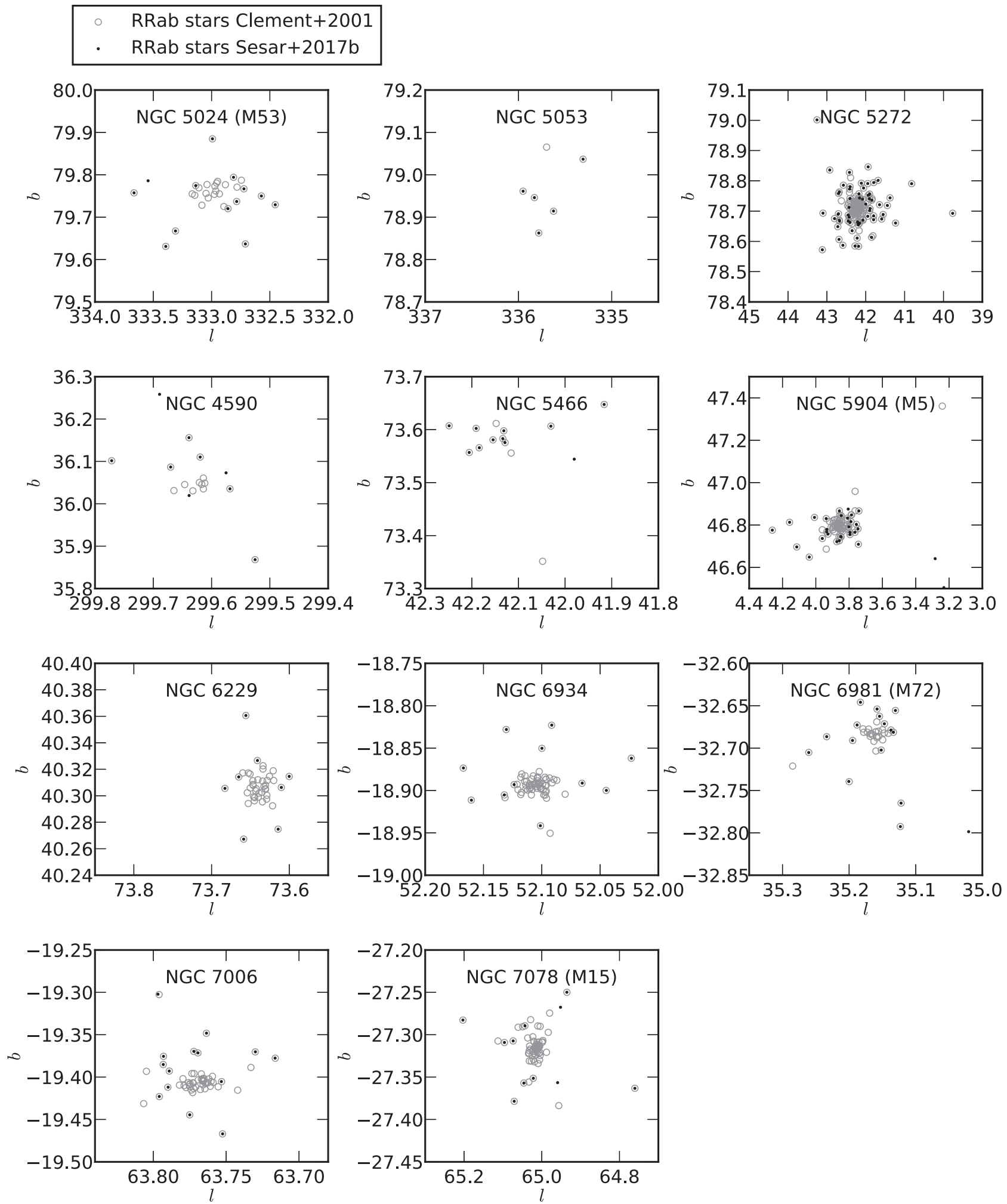

Figure 12. Comparison of the RRab stars available in our PS1 RRab catalog (Sesar et al. 2017) as used for this work, to the RRab stars in the Catalog of Variable Stars in Galactic Globular Clusters (Clement et al. 2001). For the 11 globular clusters available in both catalogs, we find that our catalog misses most RRab in the central regions of the globular clusters. These regions are too compact, and thus most stars did not pass the quality criteria for the PS1 RRab catalog. 
mock dSph with halo background
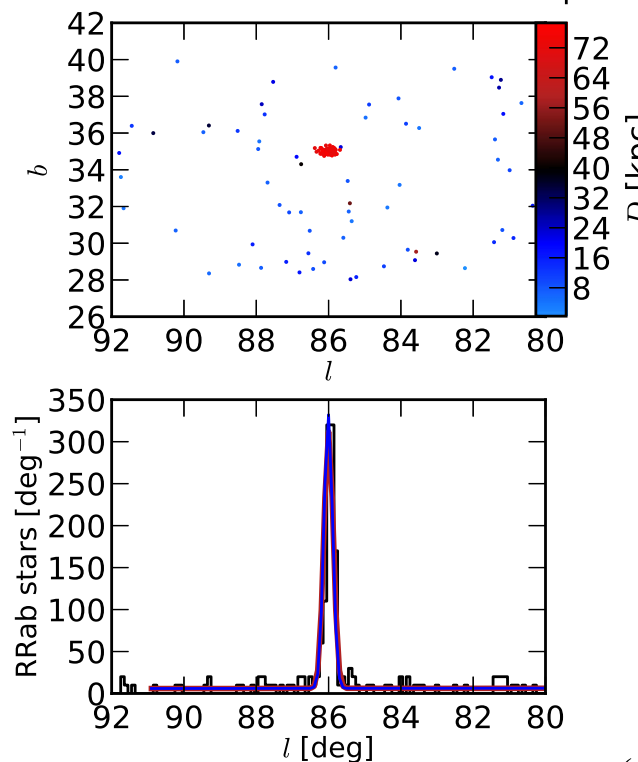

(a)
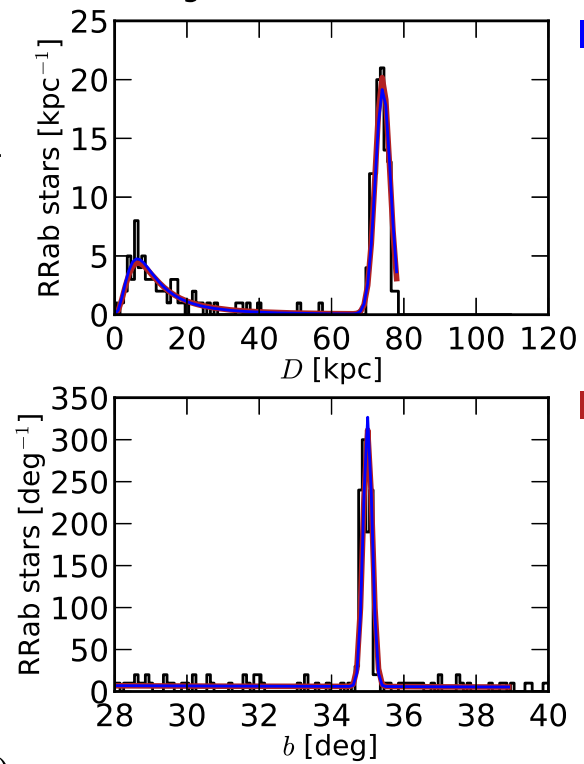

mock gc with halo background
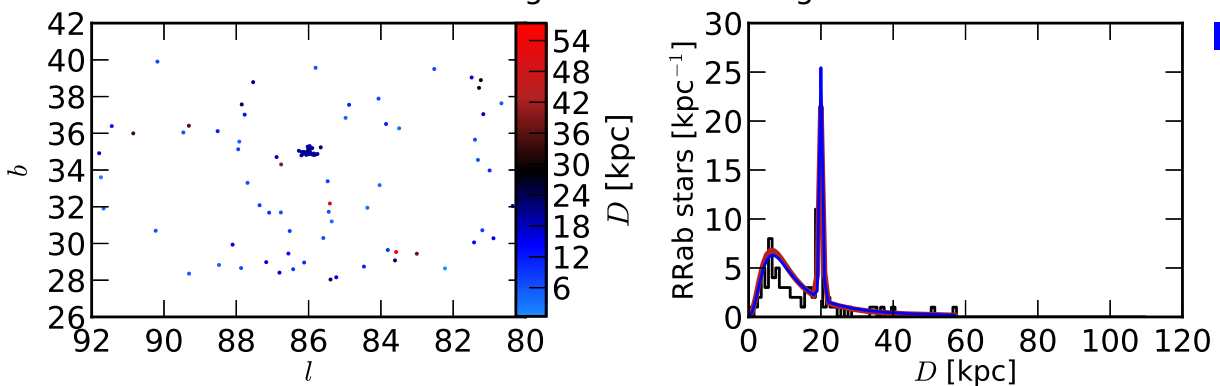

best fit parameters

with $1 \sigma$

$n=4.35_{-0.3}^{+0.25}$

$f_{*}=0.21_{-0.057}^{+0.057}$

$D=20.08_{-0.08}^{+0.12} \mathrm{kpc}$

$\sigma_{D}=0.41_{-0.09}^{+1.9} \mathrm{kpc}$

$l=86.00_{-1.2}^{+0.081} \mathrm{deg}$

$\sigma_{l}=0.14_{-0.026}^{+1.8} \mathrm{deg}$

$b=34.96_{-0.07}^{+1.4} \mathrm{deg}$

$\sigma_{b}=0.16_{-0.034}^{+0.78} \mathrm{deg}$

input parameters

$n=4.50$

$f_{*}=0.20$

$D=20.00 \mathrm{kpc}$

$\sigma_{D}=0.50 \mathrm{kpc}$

$l=86.00 \mathrm{deg}$

$\sigma_{l}=0.13 \mathrm{deg}$

$b=35.00 \mathrm{deg}$

$\sigma_{b}=0.13 \mathrm{deg}$

(b)

Figure 13. Fit to two mock overdensities, where the one in subfigure (a) resembles a typical dSph with 166 sources, and the one in subfigure (b) resembles a typical GC with 91 sources. We used mock overdensities to test the methodology for fitting overdensities, as well as estimate typical error ranges. The best-fit set of parameters along with their $1 \sigma$ intervals, as well as the input parameters used to generate the mock overdensities and background distribution of halo stars, are given in the right part of each panel. In each case, the first panel shows a map of the star distribution near the overdensity, where the stars are color-coded according to their heliocentric distance. The other three panels show the histograms in $l, b, D$ for the stars from the first panel. Overplotted are both the distribution the mock stars were drawn from (red) and the best-fit model from Section 4 (blue). We find results that are consistent with the input model within reasonable uncertainties, which means that we are able to recover the input parameters for all models in their assumed parameter range. 

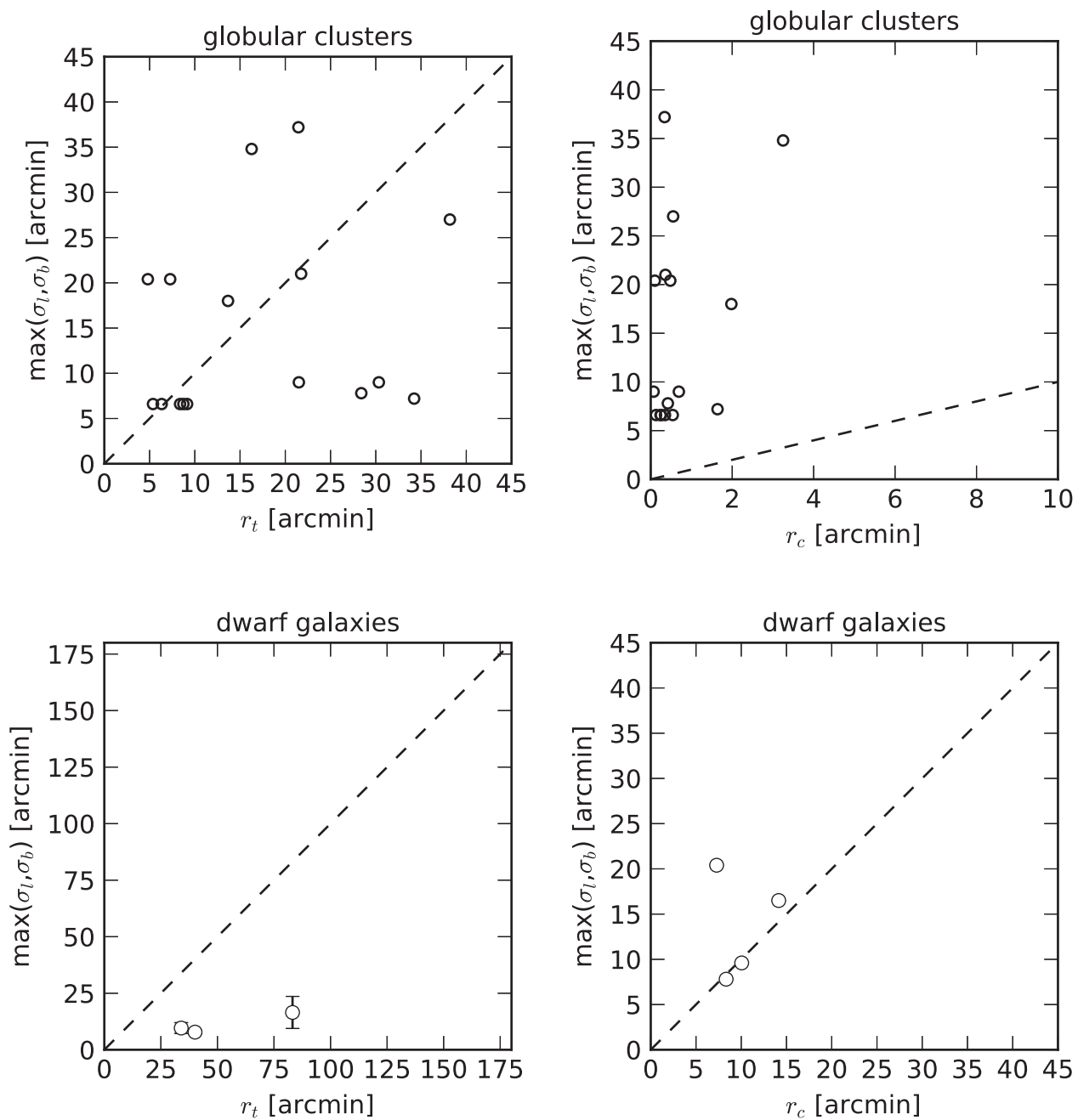

Figure 14. Comparison between the estimated extent of the overdensities (here we take the maximum of $\sigma_{l}$ and $\sigma_{b}$, max $\left(\sigma_{l}, \sigma_{b}\right)$ ) in the present study and the tidal radii $r_{t}$ and core radii $r_{c}$ for globular clusters (Harris 1996, no uncertainties are available) and for dwarf galaxies (from different sources, see Table 7; uncertainties are partially available). For all of our remote globular clusters and for three of our dwarf galaxies, we were able to look up $r_{t}, r_{c}$. The dwarf galaxy Ursa Major I lacks a published tidal and core radius. The diagonal line represents the one-to-one relation. We find that for globular clusters, whereas the distribution shows a lot of scatter, our estimated extent from $\max \left(\sigma_{l}, \sigma_{b}\right)$ represents significant fractions of the tidal radius. We find sources far beyond the core radius. For dwarf galaxies, our estimated extent from $\max \left(\sigma_{l}, \sigma_{b}\right)$ matches the core radius quite well. We do not pick up sources that are as distant as the tidal radius. Tables 7 and 8 list the data used for this figure. 


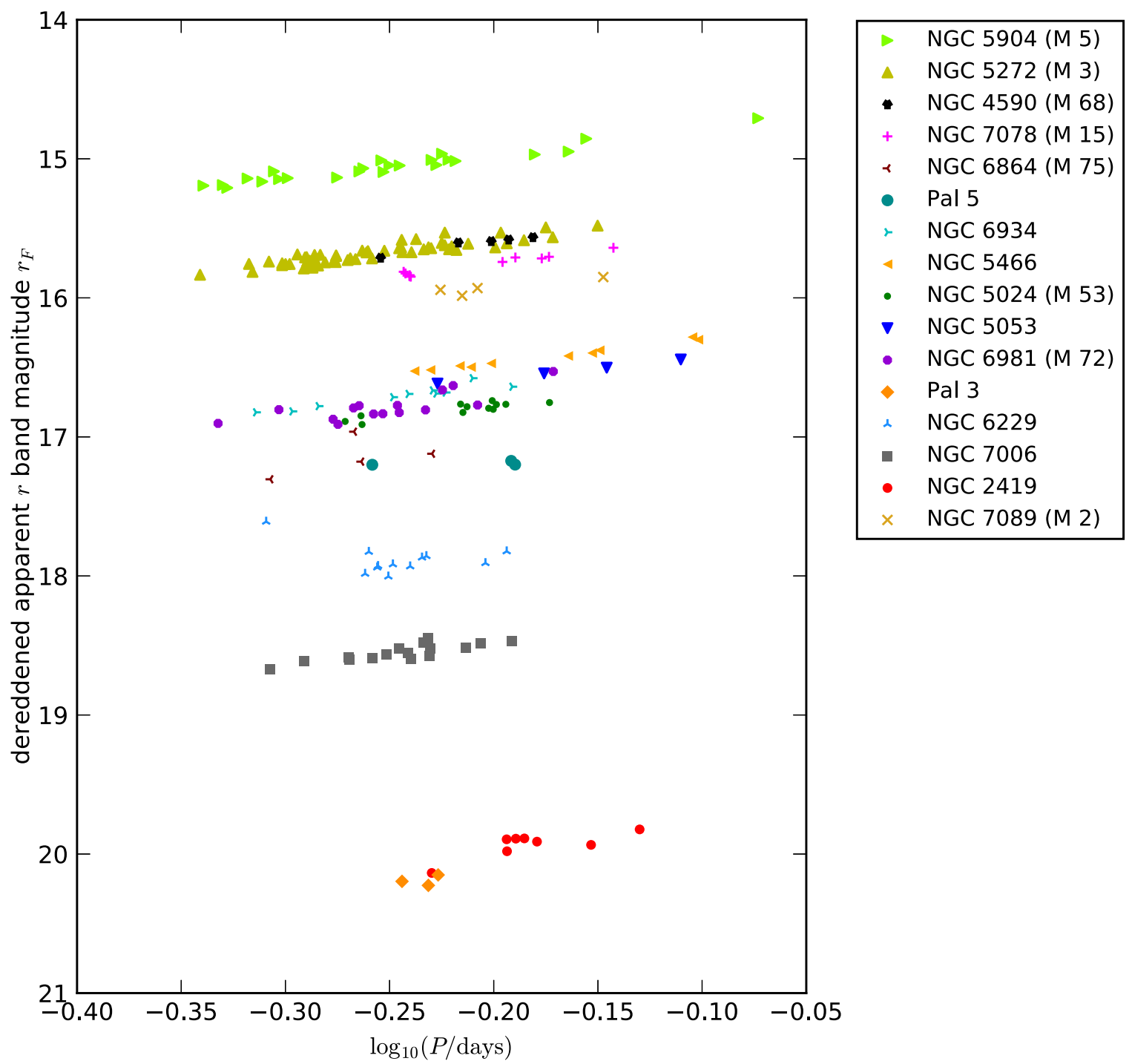

Figure 15. We plot the dereddened apparent $r$-band magnitude $\left(r_{F}\right.$ in the PS1 RRab catalog) for each of the RRab in our sample for each globular cluster vs. their period. The typical trend of a PL relation is clearly visible. 

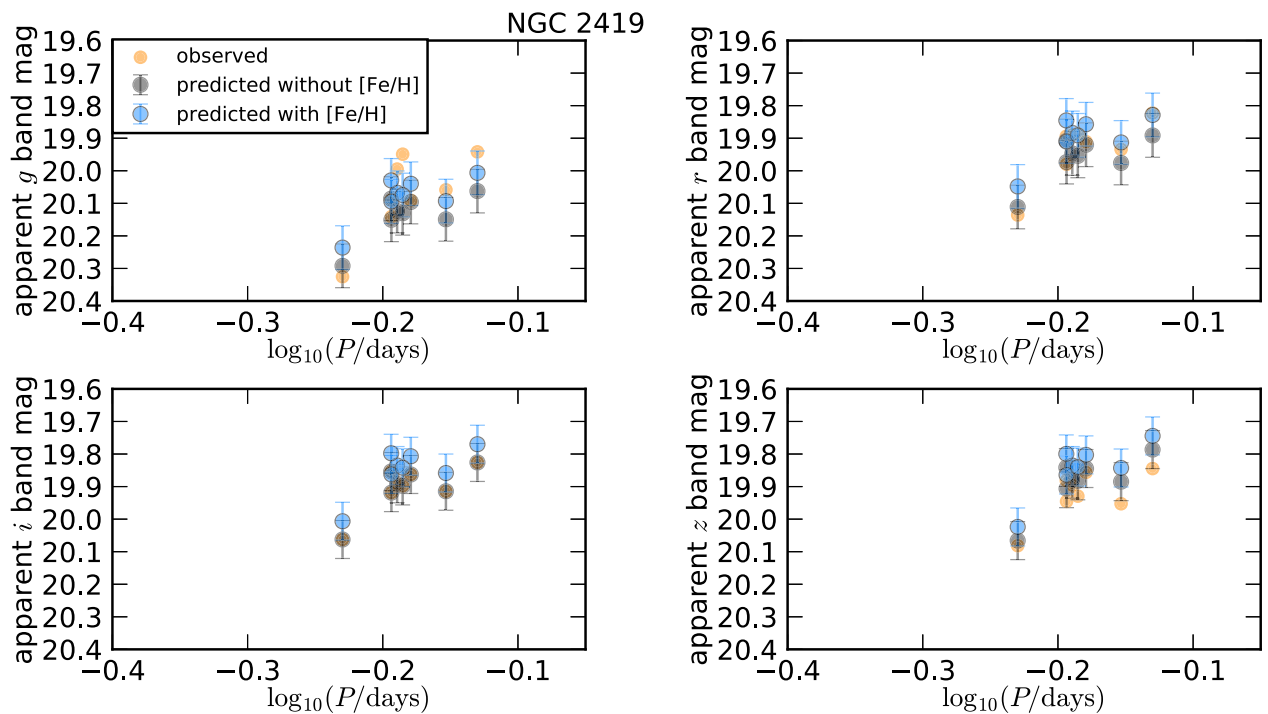

Figure 16. In this and the following plots, we select the RRab stars for each globular cluster and plot their dereddended apparent $g, r, i, z$ magnitudes $\left(g_{F}, \ldots, z_{F}\right.$ in the PS1 RRab catalog) vs. their periods. These are the orange points in each panel. Along with that, we plot the apparent magnitude one would get from the PLZ or PL relation for each of the periods. The gray points describe the predicted apparent magnitude based on period without any assumption on metallicity (Equation (13)) whereas the blue points describe the predicted apparent magnitude based on period when taking the metallicity $[\mathrm{Fe} / \mathrm{H}]$ from Equation (12) into account. We find that for most of the 16 globular clusters we have evaluated, the predicted apparent magnitude with $[\mathrm{Fe} / \mathrm{H}]$ (blue markers in the figures) is a bit brighter than the predicted apparent magnitude without $[\mathrm{Fe} / \mathrm{H}]$ (black markers), and this is again a bit brighter than the observed dereddened apparent magnitude (orange markers). This figure was made for the globular cluster NGC 2419. In the following figures, we show similar plots for all the globular clusters discussed in this paper.
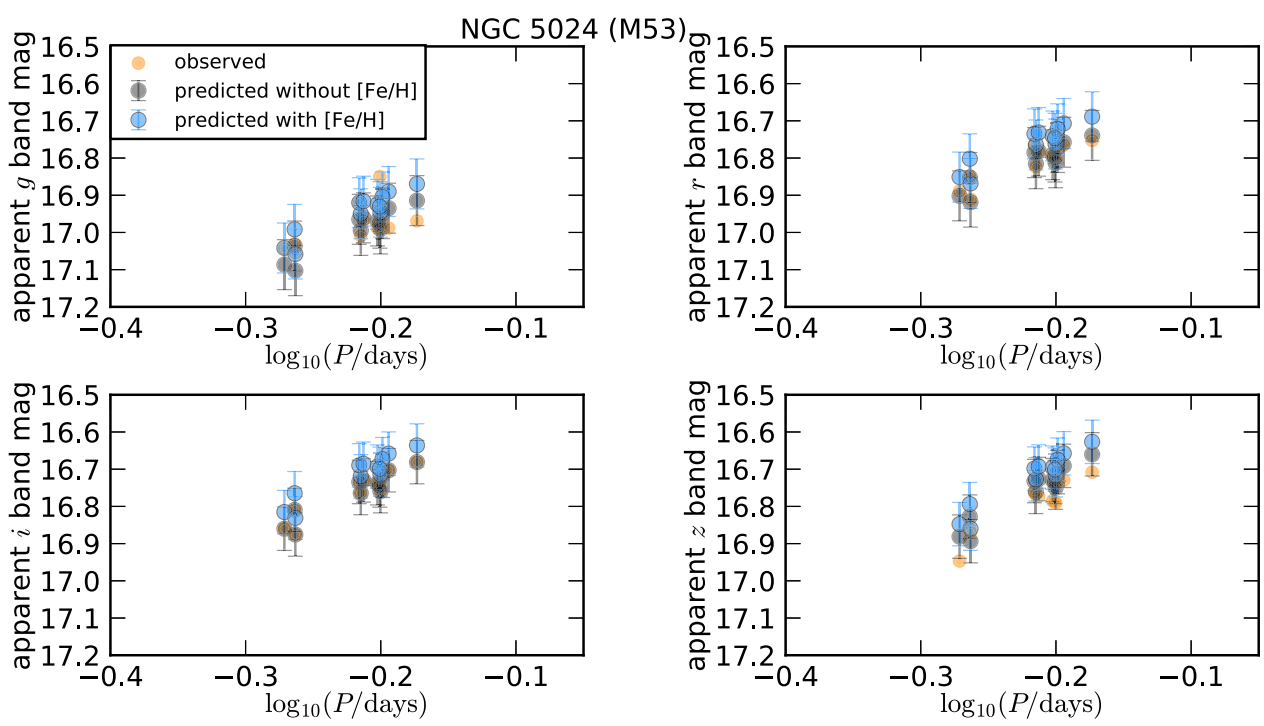

Figure 17. Observed and predicted apparent magnitudes for the globular cluster NGC 5024 (M53). See Figure 16 for a detailed description. 

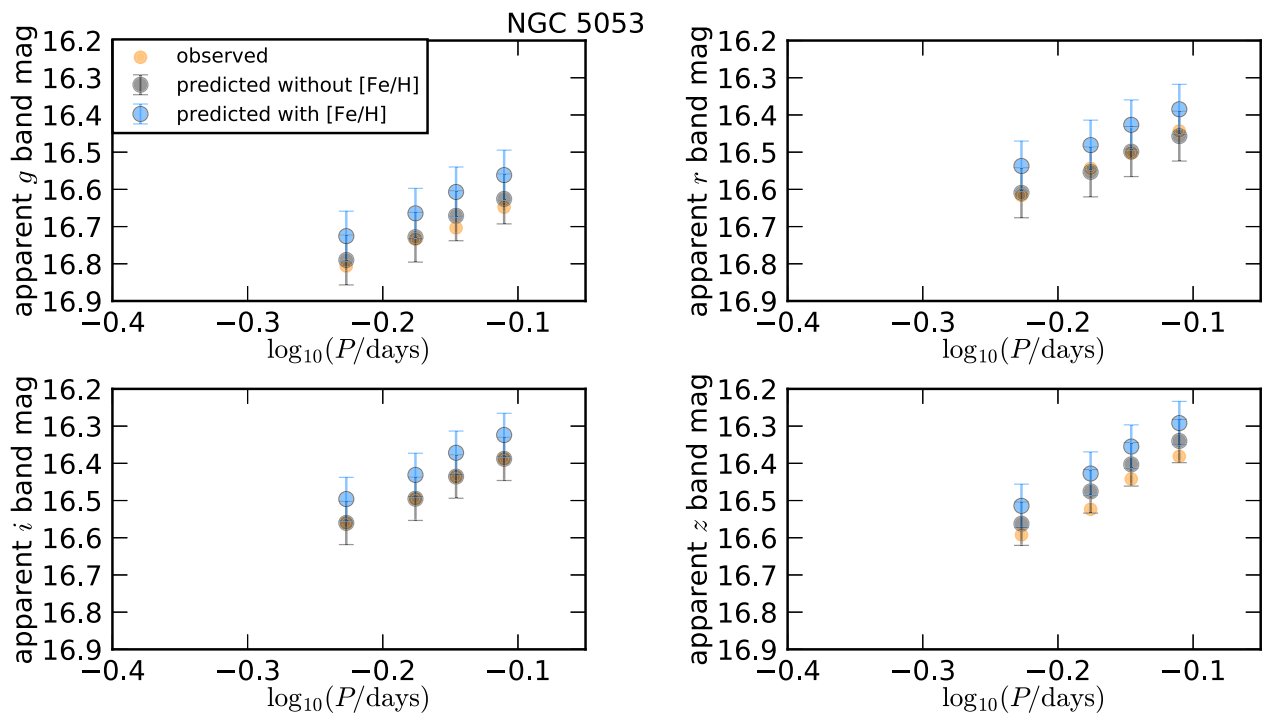

Figure 18. Observed and predicted apparent magnitudes for the globular cluster NGC 5053. See Figure 16 for a detailed description.
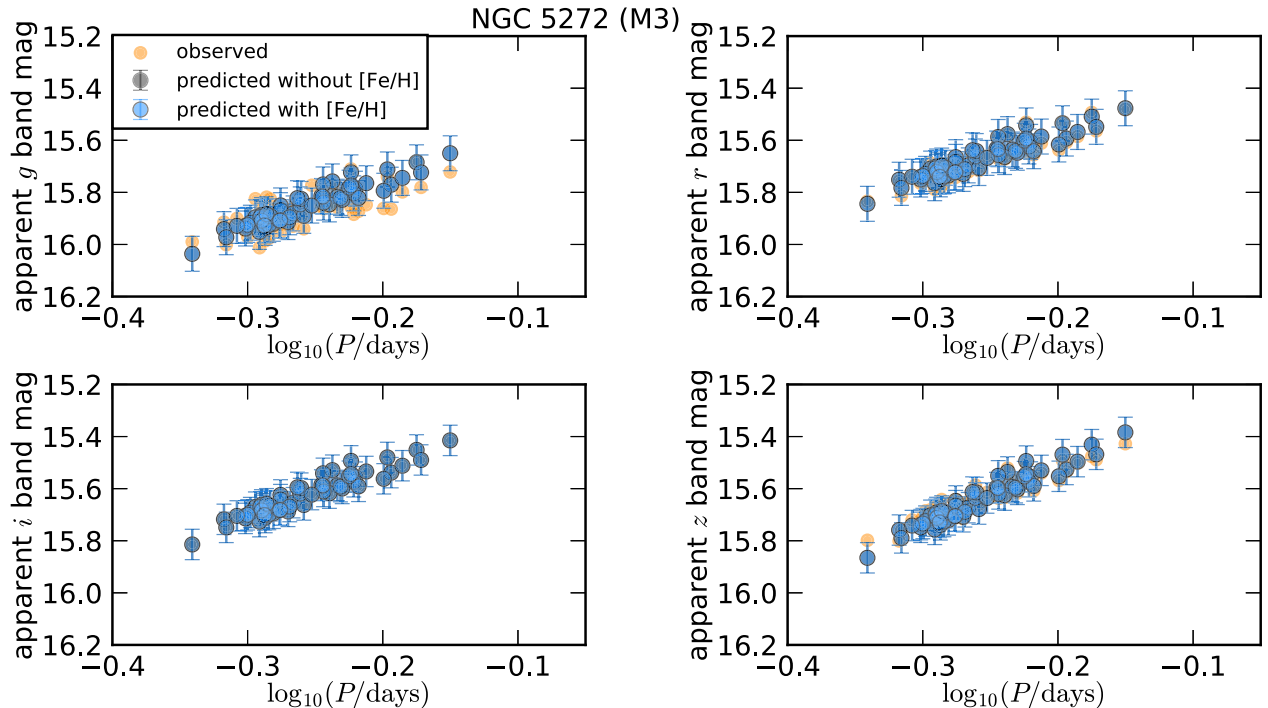

Figure 19. Observed and predicted apparent magnitudes for the globular cluster NGC 5272 (M3). See Figure 16 for a detailed description.
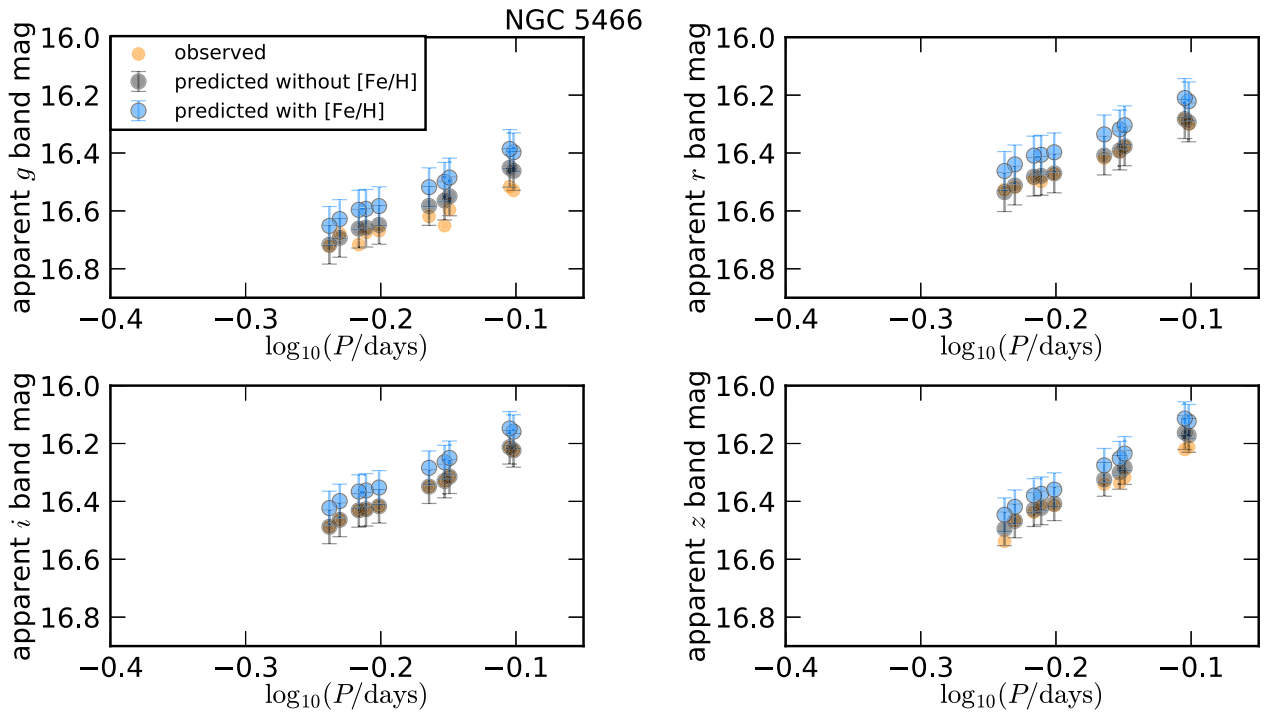

Figure 20. Observed and predicted apparent magnitudes for the globular cluster NGC 5466. See Figure 16 for a detailed description. 

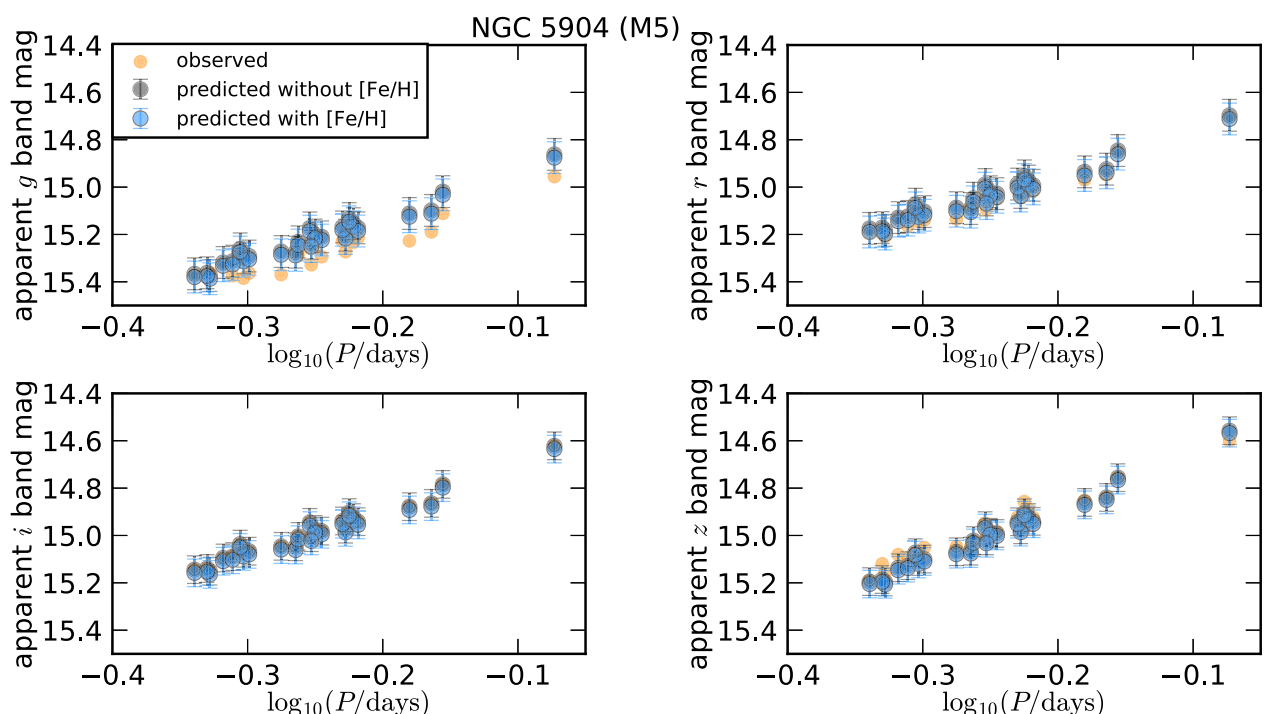

Figure 21. Observed and predicted apparent magnitudes for the globular cluster NGC 5904 (M5). See Figure 16 for a detailed description.
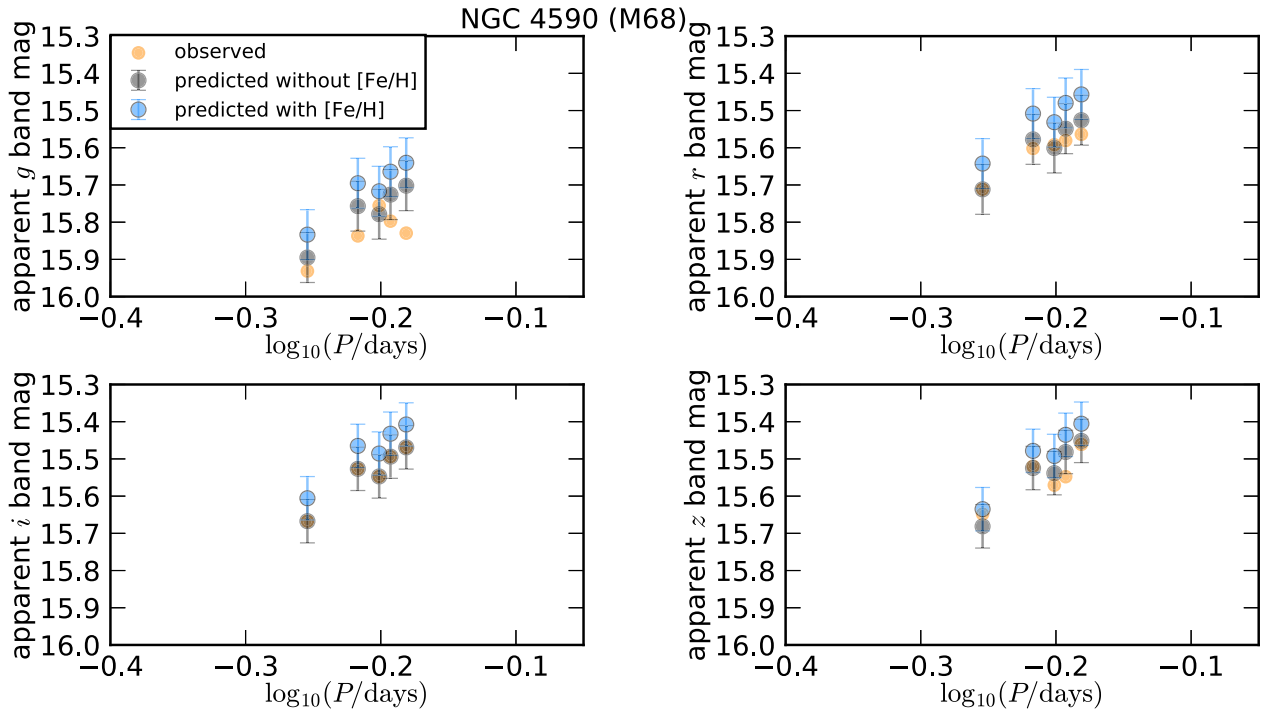

Figure 22. Observed and predicted apparent magnitudes for the globular cluster NGC 4590 (M68). See Figure 16 for a detailed description.
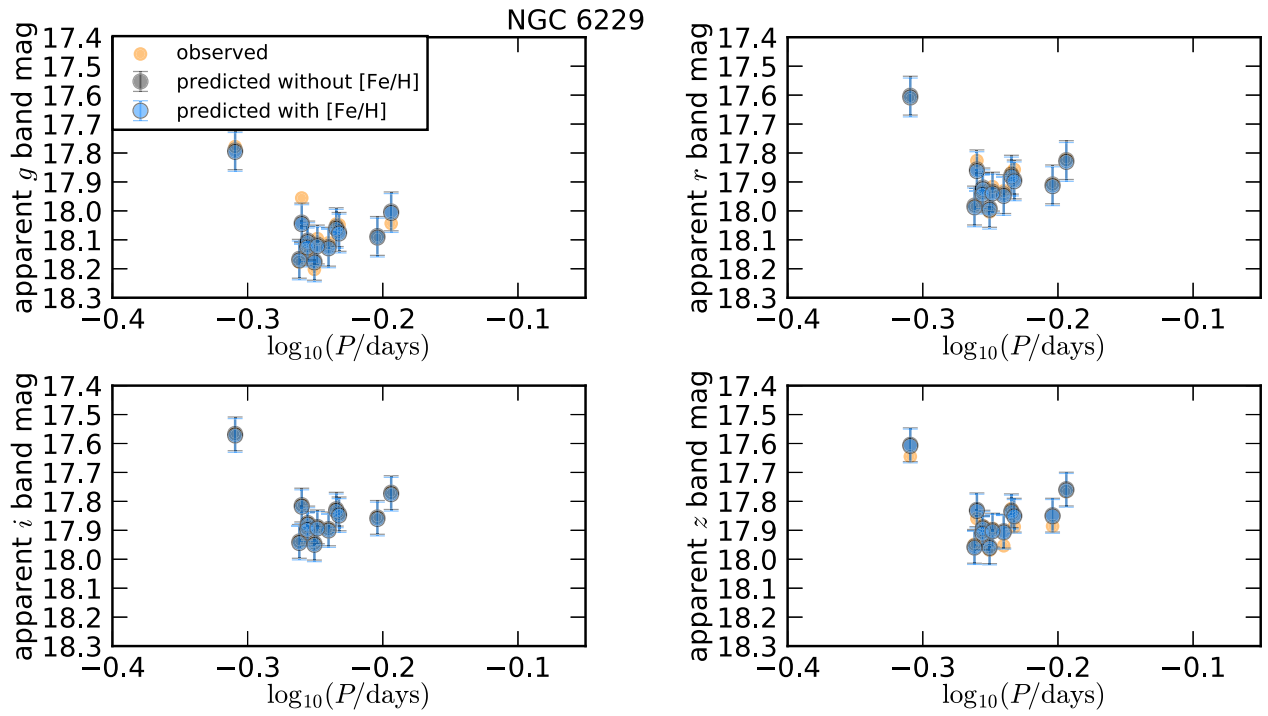

Figure 23. Observed and predicted apparent magnitudes for the globular cluster NGC 6229. See Figure 16 for a detailed description. 

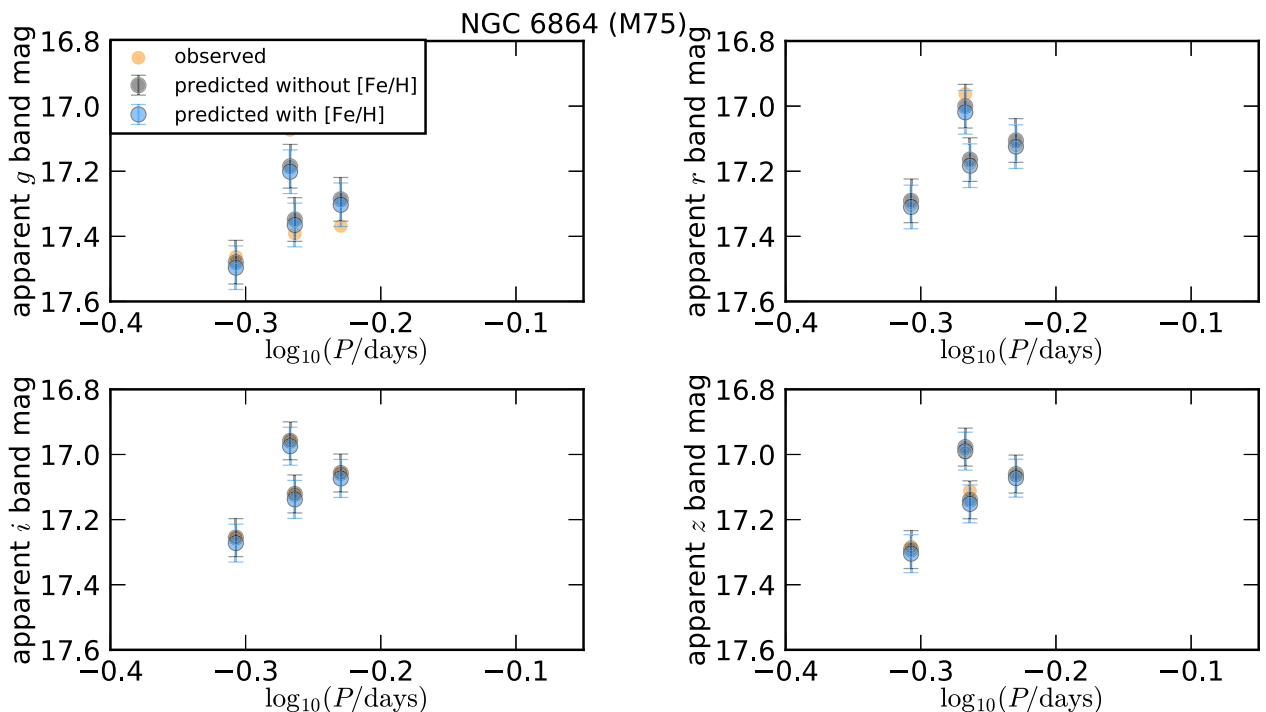

Figure 24. Observed and predicted apparent magnitudes for the globular cluster NGC 6864 (M75). See Figure 16 for a detailed description.
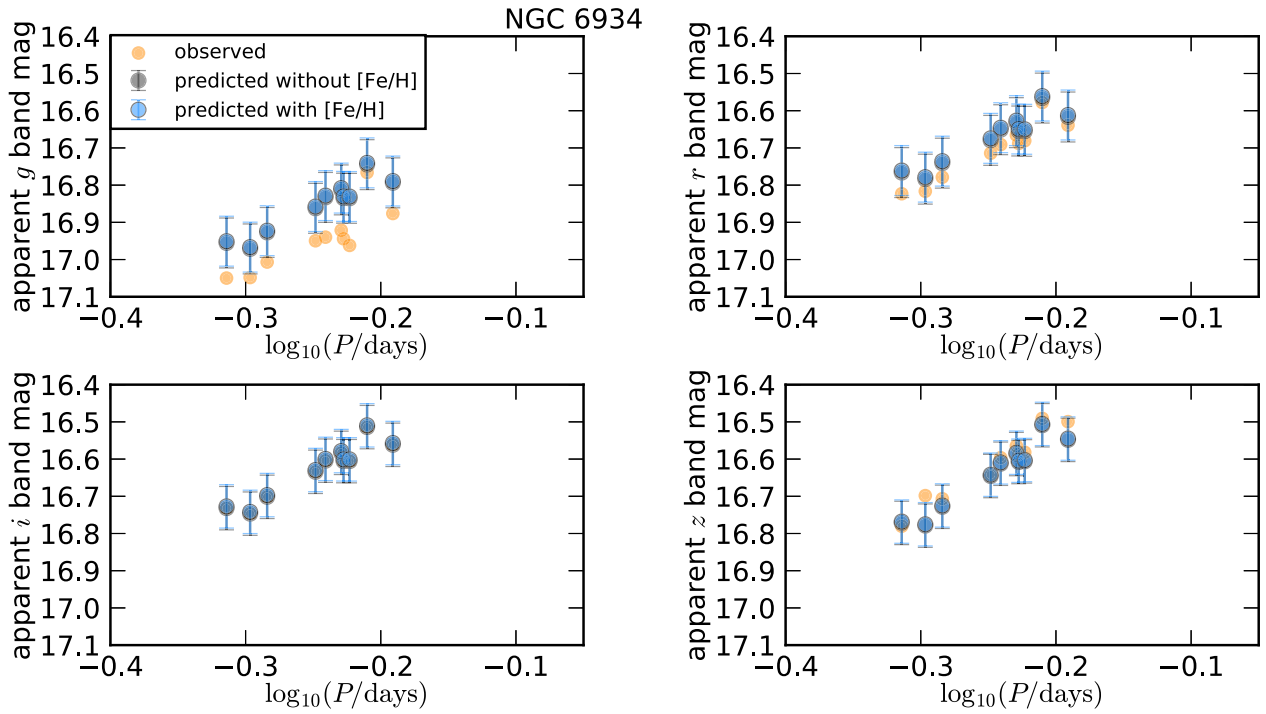

Figure 25. Observed and predicted apparent magnitudes for the globular cluster NGC 6934. See Figure 16 for a detailed description.
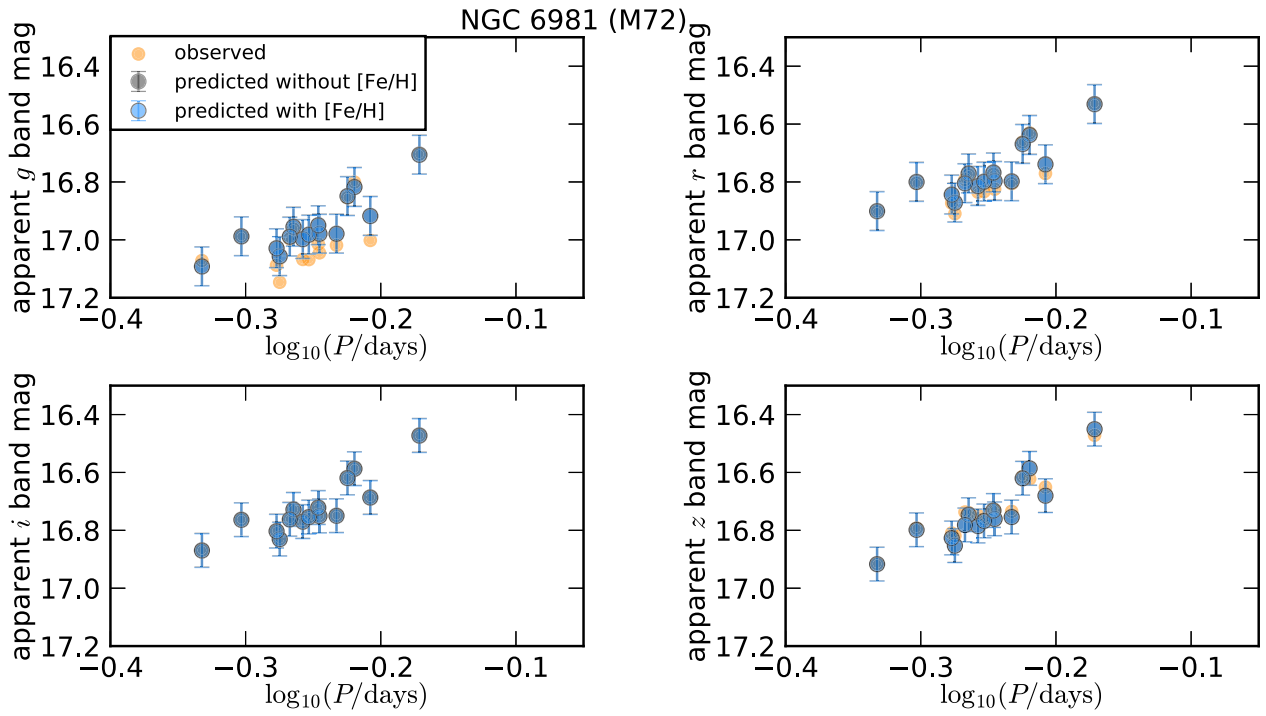

Figure 26. Observed and predicted apparent magnitudes for the globular cluster NGC 6981 (M72). See Figure 16 for a detailed description. 

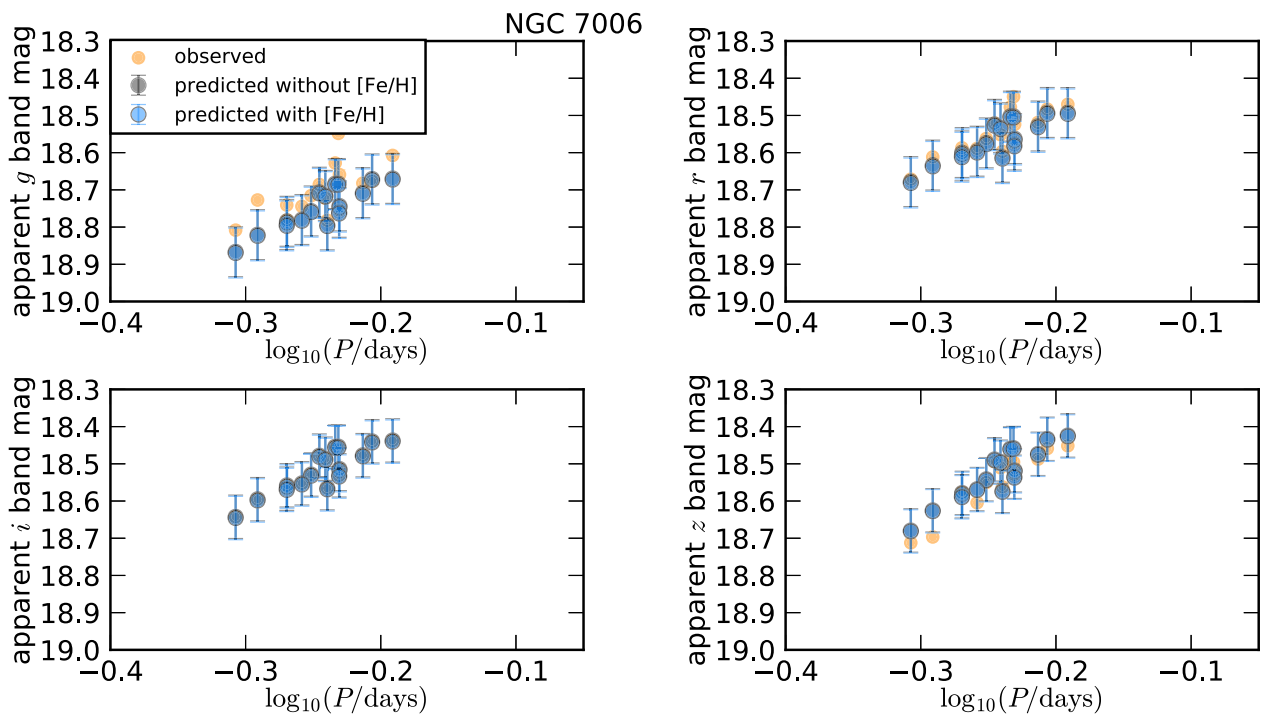

Figure 27. Observed and predicted apparent magnitudes for the globular cluster NGC 7006. See Figure 16 for a detailed description.
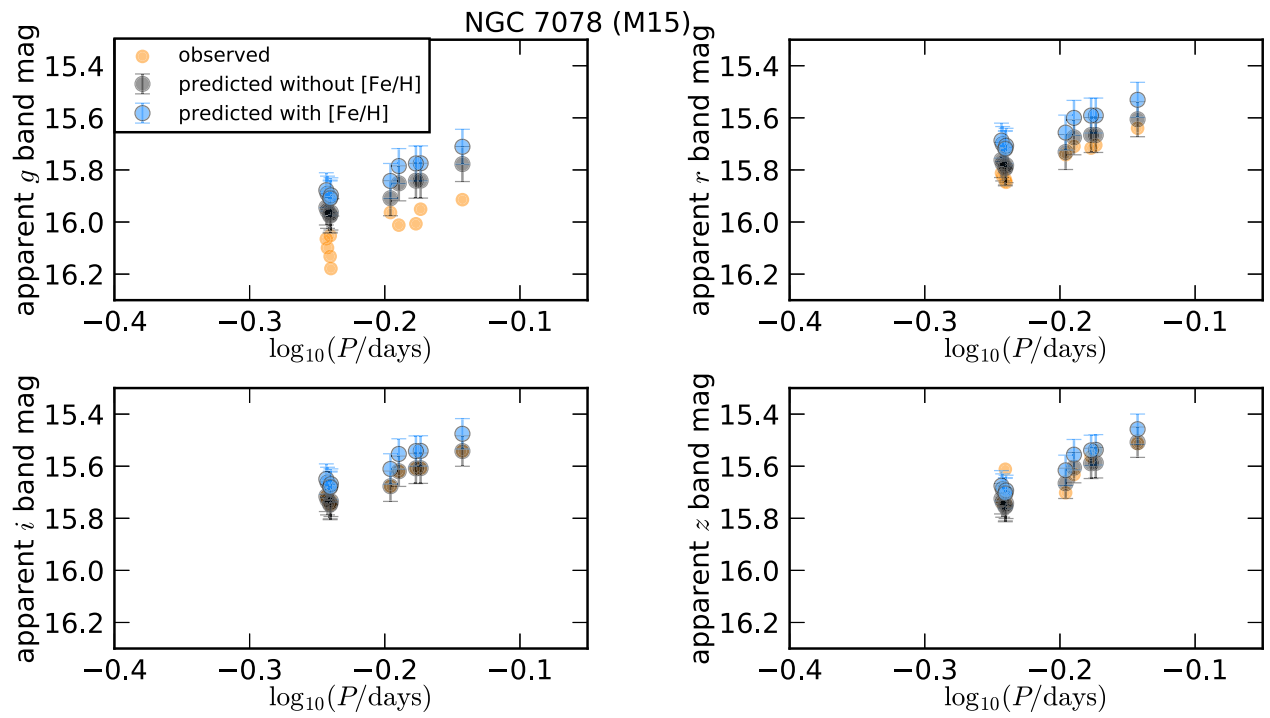

Figure 28. Observed and predicted apparent magnitudes for the globular cluster NGC 7078 (M15). See Figure 16 for a detailed description.
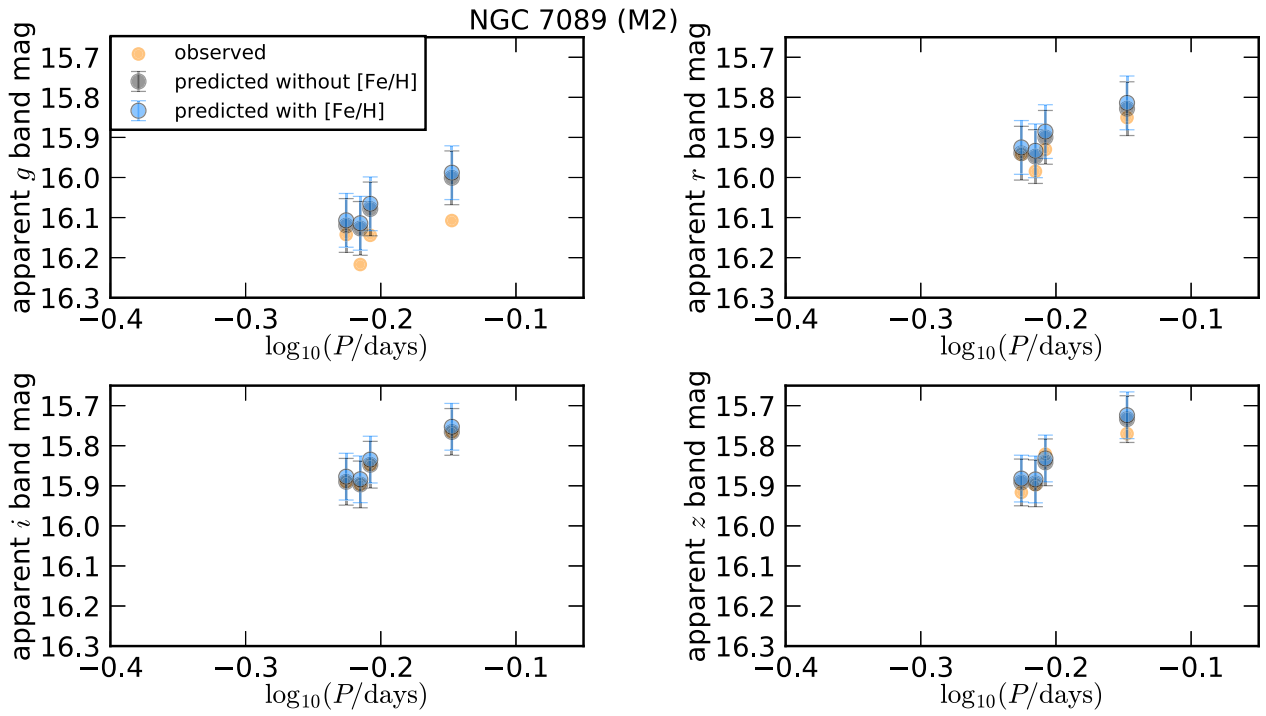

Figure 29. Observed and predicted apparent magnitudes for the globular cluster NGC 7089 (M2). See Figure 16 for a detailed description. 

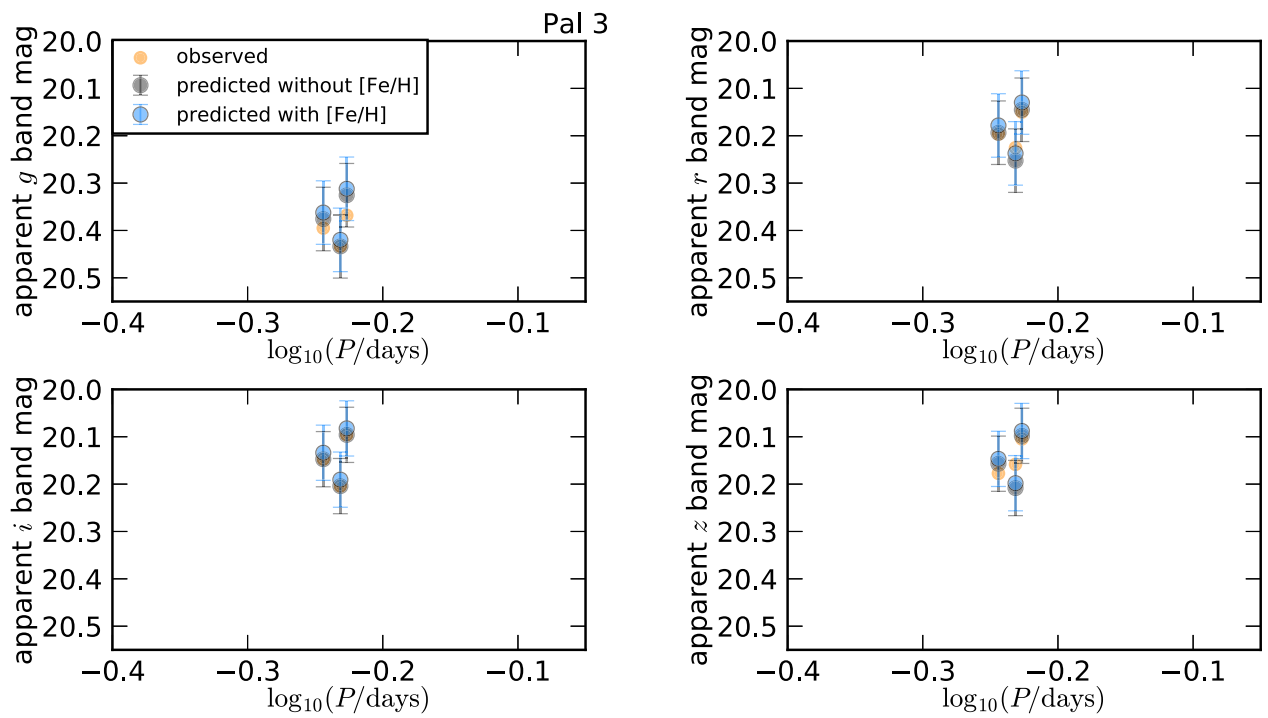

Figure 30. Observed and predicted apparent magnitudes for the globular cluster Pal 3. See Figure 16 for a detailed description.
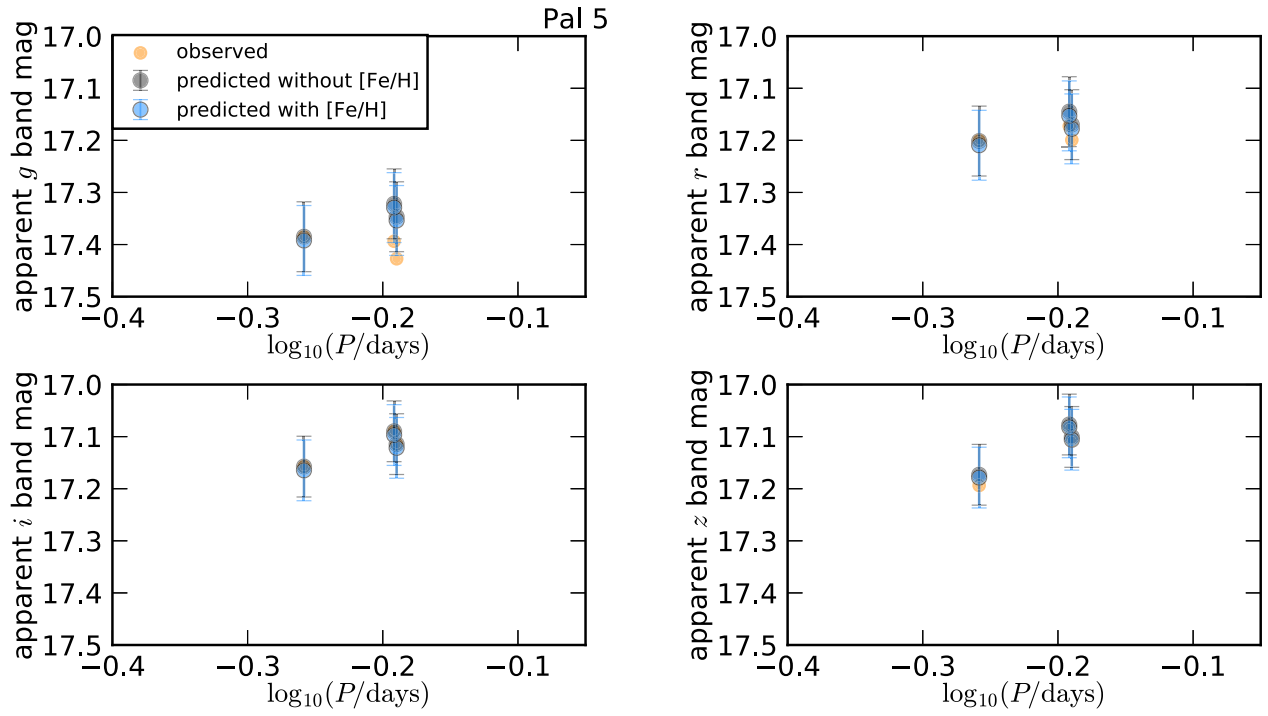

Figure 31. Observed and predicted apparent magnitudes for the globular cluster Pal 5. See Figure 16 for a detailed description. 

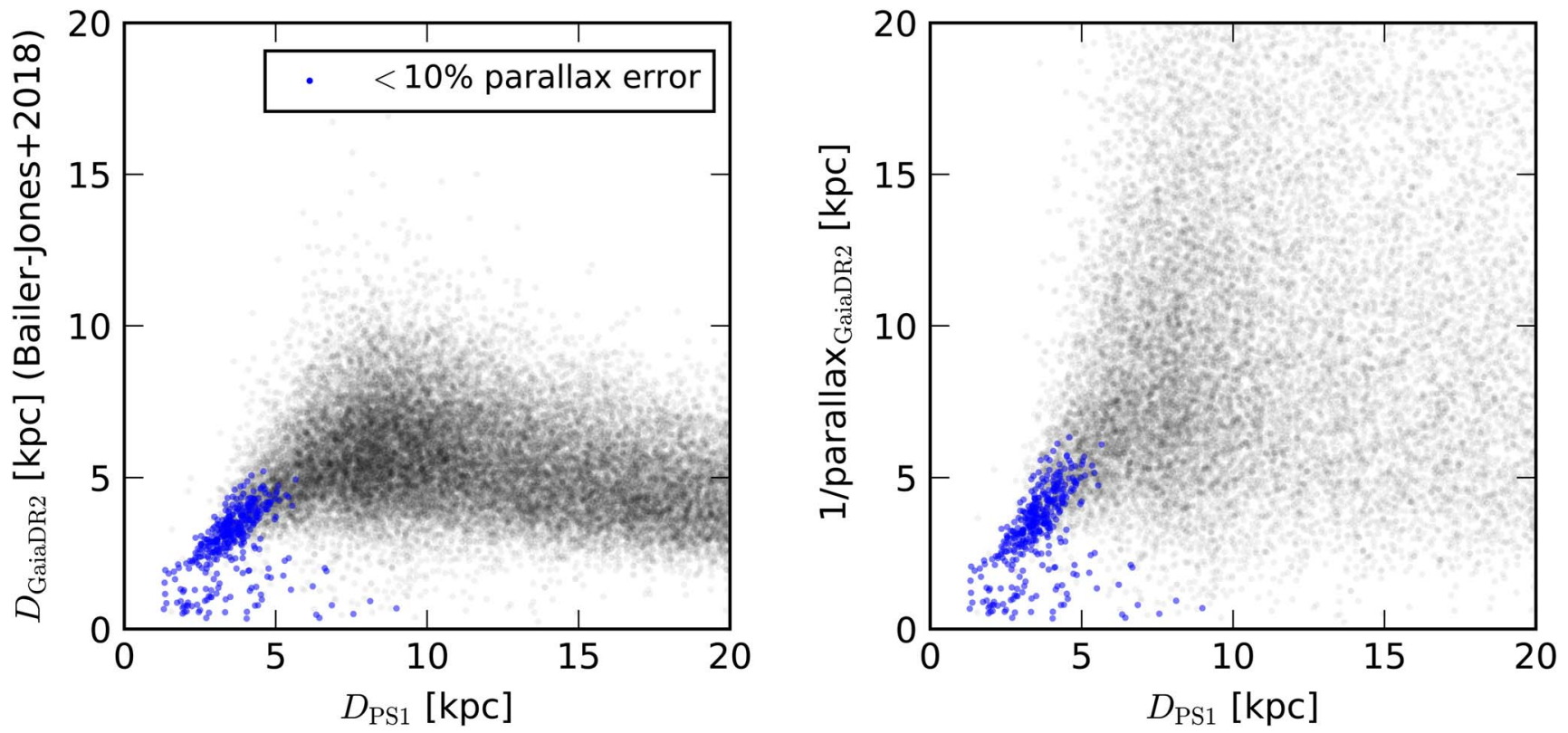

Figure 32. To compare our distance estimates $D_{\mathrm{PS} 1}$ from Sesar et al. (2017) to those of Gaia DR2 (Bailer-Jones et al. 2018), we cross-matched our PS1 RRab sample with Gaia DR2, and found a cross-match for 43,791 sources. The plot shows the $D_{\text {GaiaDR2 }}$ and a rough distance estimate $1 /$ parallax vs. $D_{\mathrm{PS} 1}$ for the RRab stars within $D_{\mathrm{PS} 1}<20 \mathrm{kpc}$. Blue points indicate RRab stars with parallax errors of $<10 \%$. We find a rather large scatter in the distances, far larger than the PS1 distance uncertainty of $3 \%$ or an estimate of the distance uncertainty from the parallax error. Beyond about $5 \mathrm{kpc}$, where the parallax error also exceeds $10 \%$, the distances from Gaia DR2 become very unreliable.

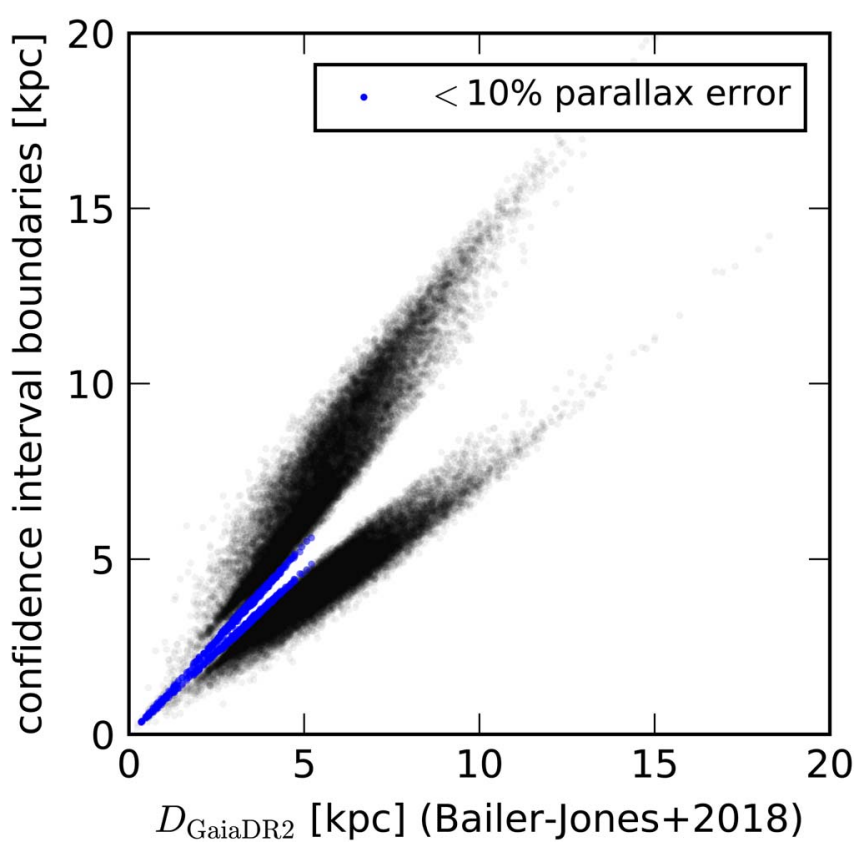

Figure 33. Bailer-Jones et al. (2018) claim quite large uncertainties of their distance estimates. The plot shows the upper and lower $68 \%$ confidence interval boundaries of their distance estimates for our cross-matched sample of RRab stars. Again, blue points indicate RRab stars with $<10 \%$ parallax error (blue points). For those RRab stars with a parallax error of $\sim 10 \%$, we find that the uncertainty is about $10 \%$, and otherwise, can increase to as much as $50 \%$ for many RRab stars.

\section{ORCID iDs}

Nina Hernitschek (i) https://orcid.org/0000-0003-1681-0430 Judith G. Cohen (ib https://orcid.org/0000-0002-8039-4673 Hans-Walter Rix (iD https://orcid.org/0000-0003-4996-9069 Eugene Magnier (iD https://orcid.org/0000-0002-7965-2815 Richard Wainscoat (1D https://orcid.org/0000-0002-1341-0952

\section{References}

Bailer-Jones, C. A. L., Rybizki, J., Fouesneau, M., Mantelet, G., \& Andrae, R. 2018, ApJ, arXiv:1804.10121

Bellazzini, M., Ferraro, F. R., Origlia, L., et al. 2002, AJ, 124, 3222

Belokurov, V., Walker, M. G., Evans, N. W., et al. 2009, MNRAS, 397, 1748, 4

Belokurov, V., Zucker, D. B., Evans, N. W., et al. 2007, ApJ, 654, 897

Bonanos, A. Z., Stanek, K. Z., Szentgyorgyi, A. H., et al. 2004, ApJ, 127, 861

Bono, G., Caputo, F., Castellani, V., et al. 2003, MNRAS, 344, 1097

Bono, G., Caputo, F., Castellani, V., Marconi, M., \& Storm, J. 2001, MNRAS, 326, 1183

Braga, V. F., Dall'Ora, M., Bono, G., et al. 2015, ApJ, 799, 165

Cacciari, C., \& Clementini, G. 2003, in Stellar Candles for the Extragalactic Distance Scale, Vol. 635 ed. D. Alloin \& W. Gieren (Berlin: Springer), 105

Carrera, R., Aparicio, A., Martínez-Delgado, D., \& Alonso-García, J. 2002, AJ, 123, 3199

Carretta, E., Bragaglia, A., Gratton, R., D’Orazi, V., \& Lucatello, S. 2009, A\&A, 508, 695

Catelan, M., Pritzl, B. J., \& Smith, H. A. 2004, ApJS, 154, 633

Chaboyer, B., Demarque, P., \& Sarajedini, A. 1996, ApJ, 459, 558

Chambers, K. C., Magnier, E. A., Metcalfe, N., et al. 2016, arXiv: 1612.05560

Clement, Ch. M., Muzzin, A., Dufton, Q., et al. 2001, AJ, 122, 2587

Cohen, J. G., Sesar, B., Banholzer, S., et al. 2015, in Proc. IAU Symp. 317, The General Assembly of Galaxy Halos: Structure, Origin and Evolution, ed. A. Bragaglia et al. (Cambridge: Cambridge University Press), 91

Dall'Ora, M., Kinemuchi, K., Ripepi, V., et al. 2012, ApJ, 752, A42

Dambis, A. K., Berdnikov, L. N., Kniazev, A. Y., et al. 2013, MNRAS, 435, 3206

Foreman-Mackey, D., Hogg, D. W., Lang, D., et al. 2013, PASP, 125, 306

Gaia Collaboration 2018, A\&A, arXiv:1804.09365

Garofalo, A., Cusano, F., Clementini, G., et al. 2013, ApJ, 767, A62

Garofalo, A., Scowcroft, V., Clementini, G., et al. 2018, MNRAS, 481, 578

Goodman, J., \& Weare, J. 2010, Communications in Applied Mathematics and Computational Science, 5, 81

Hammer, F., Yang, Y., Arenou, F., et al. 2018, ApJ, 860, 1

Harris, W. E. 1996, AJ, 112, 1487

Hernitschek, N., Cohen, J. G., Rix, H.-W., et al. 2018, ApJ, 859, 31

Hernitschek, N., Rix, H.-W., Schlafly, E. F., et al. 2016, ApJ, 817, 73

Hernitschek, N., Sesar, B., Rix, H.-W., et al. 2017, ApJ, 850, 96

Iorio, G., Belokurov, V., Erkal, D., et al. 2017, MNRAS, 474, 2142

Irwin, M., \& Hatzidimitriou, D. 1995, MNRAS, 277, 1254

Joo, S. J., Kyeong, J., Yang, S. C., et al. 2018, ApJ, 861, 23

Kaiser, N., Burgett, W., Chambers, K., et al. 2010, Proc. SPIE, 7733, 77330E 
Klein, C. R., Richards, J. W., Butler, N. R., \& Bloom, J. S. 2014, MNRAS, 440, L96

Kleyna, J. T., Geller, M. J., Kenyon, S. J., et al. 1998, AJ, 115, 2359

Longmore, A. J., Fernley, J. A., \& Jameson, R. F. 1986, MNRAS, 220, 279

Madore, B. F., Hoffman, D., Freedman, W. L., et al. 2013, ApJ, 776, 135

Martin, N. F., de Jong, J. T. A., \& Rix, H.-W. 2008, ApJ, 684, 1075

McConnachie, A. W. 2012, AJ, 144, 4

Medina, G. E., Muñoz, R. R., Vivas, A. K., et al. 2018, ApJ, 855, 43

Monaco, L., Bellazzini, M., Ferraro, F. R., \& Pancino, E. 2004, MNRAS, 353,874

Muraveva, T., Garofalo, A., Scowcroft, V., et al. 2018b, MNRAS, 480, 4138

Neeley, J. R., Marengo, M., Bono, G., et al. 2015, ApJ, 808, 11

Neeley, J. R., Marengo, M., Bono, G., et al. 2017, ApJ, 841, 84

Odenkirchen, M., Grebel, E. K., Harbeck, D., et al. 2001, AJ, 122, 2538

Okamoto, S., Arimoto, N., Yamada, Y., \& Onodera, M. 2008, A\&A, 487, 103

Okamoto, S., Arimoto, N., Yamada, Y., \& Onodera, M. 2012, ApJ, 844, A96

Roderick, T. A., Jerjen, H., Da Costa, G. S., et al. 2016, MNRAS, 460, 30

Schlafly, E. F., \& Finkbeiner, D. P. 2011, ApJ, 737, 103
Schlafly, E. F., Green, G., Finkbeiner, D. P., et al. 2014, ApJ, 789, 15

Sesar, B., Hernitschek, N., Mitrović, S., et al. 2017, AJ, 153, 204

Sesar, B., Ivezić, Ž., Scott, J. S., et al. 2013, AJ, 146, 21

Siegel, M. H. 2006, ApJ, 649, 2

Siegel, M. H., Majewski, S. R., Cudworth, K. M., \& Takamiya, M. 2010, AJ, 121,935

Simon, J. D., \& Geha, M. 2007, ApJ, 670, 313

Smith, H. A. 2004, RR Lyrae Stars (Cambridge: Cambridge Univ. Press)

Sollima, A., Cacciari, C., \& Valenti, E. 2006, MNRAS, 372, 1675

Stoehr, F. F., White, S. D. M., Tormen, G., et al. 2002, MNRAS, 355, L84

Stubbs, C. W., Doherty, P., Cramer, C., et al. 2010, ApJS, 191, 376

Tonry, C. W., Stubbs, K. R., Lykke, K. R., et al. 2012, ApJ, 750, 99

Torrealba, G., Koposov, S. E., Belokurov, V., \& Irwin, M. 2016a, MNRAS, 459,2370

Torrealba, G., Koposov, S. E., Belokurov, V., et al. 2016b, MNRAS, 463,712

van den Bergh, S. 1999, A\&ARv, 9, 273

Xue, X.-X., Rix, H.-W., Zhibo, M., et al. 2015, ApJ, 809, 144 